\title{
The iEBE-VISHNU code package for relativistic heavy-ion collisions
}

\author{
Chun Shen ${ }^{\mathrm{ab}, \text {, }}$, Zhi Qiu ${ }^{\mathrm{a}}$, Huichao Song ${ }^{\mathrm{a}, \mathrm{c}}$, Jonah Bernhard ${ }^{\mathrm{d}}$, Steffen Bass ${ }^{\mathrm{d}}$, Ulrich Heinz ${ }^{\mathrm{a}}$ \\ ${ }^{a}$ Department of Physics, The Ohio State University, Columbus, Ohio 43210-1117, USA \\ ${ }^{b}$ Department of Physics, McGill University, 3600 University Street, Montreal, Quebec, H3A 2T8, Canada \\ ${ }^{c}$ Department of Physics and State Key Laboratory of Nuclear Physics and Technology, Peking University, Beijing \\ 100871, China \\ ${ }^{d}$ Department of Physics, Duke University, Durham, North Carolina 27708, USA
}

\begin{abstract}
The iEBE-VISHNU code package performs event-by-event simulations for relativistic heavy-ion collisions using a hybrid approach based on (2+1)-dimensional viscous hydrodynamics coupled to a hadronic cascade model. We present the detailed model implementation, accompanied by some numerical code tests for the package. iEBE-VISHNU forms the core of a general theoretical framework for model-data comparisons through large scale Monte-Carlo simulations. A numerical interface between the hydrodynamically evolving medium and thermal photon radiation is also discussed. This interface is more generally designed for calculations of all kinds of rare probes that are coupled to the temperature and flow velocity evolution of the bulk medium, such as jet energy loss and heavy quark diffusion.
\end{abstract}

Keywords: Relativistic heavy-ion collision, relativistic viscous hydrodynamics, quark-gluon plasma

${ }^{*}$ Corresponding author

Email addresses: chunshen@physics.mcgill . ca (Chun Shen), heinz@mps . ohio-state.edu (Ulrich Heinz) Preprint submitted to Computer Physics Communications

July 23, 2015 


\section{PROGRAM SUMMARY}

Manuscript Title: The iEBE-VISHNU code package for relativistic heavy-ion collisions Authors: Chun Shen, Zhi Qiu, Huichao Song, Jonah Bernhard, Steffen Bass, Ulrich Heinz Program Title: iEBE-VISHNU

Journal Reference:

Catalogue identifier:

Licensing provisions: none

Programming language: Fortran, $\mathrm{C}++$, python, bash, SQLite

Computer: Laptop, desktop, cluster

Operating system: Tested on GNU/Linux Ubuntu 12.04 x64, Red Hat Linux 6, Mac OS X $10.8+$

RAM: $2 \mathrm{G}$ bytes

Number of processors used: 1

Keywords: Relativistic viscous hydrodynamics, quark-gluon plasma, Monte-Carlo simulation

Classification: 17 Nuclear Physics

External routines/libraries: GNU Scientific Library (GSL), HDF5, Numpy, UrQMD v3.3

Nature of problem:

Relativistic heavy-ion collisions are tiny in size $\left(V \sim 10^{-42} \mathrm{~m}^{3}\right)$ and live in a flash $\left(\sim 5 \times 10^{-23} \mathrm{~s}\right)$. It is impossible to use external probes to study the properties of the quark-gluon plasma (QGP), a novel state of matter created during the collisions. Experiments can only measure the momentum information of stable hadrons, who are the remnants of the collisions. In order to extract the thermal and transport properties of the QGP, one needs to rely on Monte-Carlo event-by-event model simulations, which reverse-engineer the experimental measurements to the early time dynamics of the relativistic heavy-ion collisions.

Solution method:

Relativistic heavy-ion collisions contain multiple stages of evolution. The physics that governs each stage is implemented into individual code component. A general driver script glues all the modular packages as a whole to perform large-scale Monte-Carlo simulations. The final results are stored into SQLite database, which supports standard querying for massive data analysis. By tuning transport coefficients of the QGP as free parameters, e.g. the specific shear viscosity $\eta / s$, we can constrain various transport properties of the QGP through model-data comparisons.

Running time:

The following running time is tested on a laptop computer with a $2.4 \mathrm{GHz}$ Intel Core i5 CPU, 4GB memory. All the $\mathrm{C}++$ and Fortran codes are compiled with the GNU Compiler Collection (GCC) 4.9.2 and -03 optimization.

\begin{tabular}{c|c|c|c}
\hline \hline & $\mathrm{p}+\mathrm{p}$ & $0-5 \% \mathrm{p}+\mathrm{Pb}$ & $0-5 \% \mathrm{~Pb}+\mathrm{Pb}$ \\
\hline $\begin{array}{c}\text { initial condition generator superMC } \\
(100 \text { events, } 400 \times 400 \text { grid })\end{array}$ & $20 s$ & $20 s$ & $50 s$ \\
\hline $\begin{array}{c}(2+1)-\mathrm{d} \text { hydrodynamics VISHNew } \\
(1 \text { event, } 400 \times 400 \text { grid })\end{array}$ & $120 s$ & $200 s$ & $690 s$ \\
\hline $\begin{array}{c}\text { Cooper-Frye freeze-out iSS } \\
(500 \text { events, }|y|<4)\end{array}$ & $4 s$ & $15 s$ & $350 s$ \\
\hline $\begin{array}{c}\text { hadron cascade UrQMD } \\
(1 \text { event, }|y|<4)\end{array}$ & $0.03 s$ & $0.18 s$ & $150 s$ \\
\hline \hline
\end{tabular}

Table 1: Summary of typical running time (in second) of individual component in the package. Different types of collisions are simulated at $\sqrt{s_{\mathrm{NN}}}=5.02 \mathrm{TeV}$. 


\section{Introduction}

The Relativistic Heavy-Ion Collider (RHIC) at Brookhaven National Laboratory and the Large Hadron Collider (LHC) at CERN provide unique experimental access to a new state of matter at extremely high densities and temperatures: the Quark Gluon Plasma (QGP), in which quarks and gluons are no longer confined inside individual nucleons. Studying the thermodynamic and transport properties of the QGP will help us understand emergent phenomena in hot and dense many-body systems governed by the strong interaction. However, these "little bangs" are almost point-like in size $\left(V \sim 10^{-42} \mathrm{~m}^{3}\right)$ and disappear almost instantaneously $\left(\sim 5 \times 10^{-23} \mathrm{~s}\right)$. This makes it impossible to use external probes to measure the properties of the QGP. In order to extract the dynamical evolution of relativistic heavy-ion collisions, one has to rely on realistic theoretical model simulations, which back trace the final experimental observables to the early stage of the collisions.

The evolution of a relativistic heavy-ion collision contains multiple stages which are governed by different underlying physics. Right after the initial overlap of the colliding nuclei, the system is dominated by gluons characterized by an over populated phase-space distribution [1]. The number of gluons is of order $\sim \frac{1}{g^{2}}$ with $g<1$ and these gluons carry each a very small fraction of the longitudinal momentum of the incoming nucleus (small- $x$ gluons). During the first 1 $\mathrm{fm} / c$, due to the large occupation number of gluons at leading order in strong coupling $g$, these saturated small- $x$ gluons will evolve according to the classical Yang-Mills equation of motion. It is believed that the next-to-leading order quantum corrections to the classical field evolution drive the system rapidly towards local isotropy in momentum space [2, 3] and somewhat later to local thermal equilibrium. After $0.3-0.5 \mathrm{fm} / c$, the system achieves approximately local momentum isotropy; local thermal equilibrium is reached after a few $\mathrm{fm} / c$. The quarks and gluons that are produced after the collision form a strongly coupled plasma (QGP). The dynamics of the QGP can be described by macroscopic viscous hydrodynamics where the viscous corrections account for the remaining deviation from local isotropy and thermal equilibrium [4-12]. As the system expands and cools, it will smoothly cross over from the QGP phase to a hadron gas phase according to the equation of state (EOS) determined from Lattice QCD calculations [13-17]. At hadronization, the quark-gluon fluid will convert into hadrons due to confinement. In the hadronic phase, the hadron cascade model can provide us with a detailed microscopic description of the evolution [18, 19].

As the fireball continues to expand and cool, the collision rates between the hadronic resonances decrease. First, the inelastic collisions between particles cease and the system reaches chemical freeze-out almost directly after hadronization [20]. After this point, only resonance decays and baryon-antibaryon annihilation can change the particle yields [21]. Regeneration of baryon-antibaryon pairs is a rare process that can be neglected. As the system evolves further, the density of the fireball becomes so low that the mean free time of the particles becomes much larger than the Hubble time (i.e. the time over which the inter particle spacing doubles.) [22]. The particles reach kinetic freeze out and subsequently free-stream to the detectors. In Fig. 1 we schematically summarize the theoretical models and the corresponding codes that we will use to simulate the different stages of heavy-ion collisions. We will explain them in detail in the following Sections.

In relativistic heavy-ion collisions, rare electromagnetic observables like photons and dileptons only interact with the medium through the electromagnetic interaction, which is much weaker than the strong interaction. For this reason, their mean free path is much longer than the system size, and hence they suffer negligible final state interactions after they are produced 


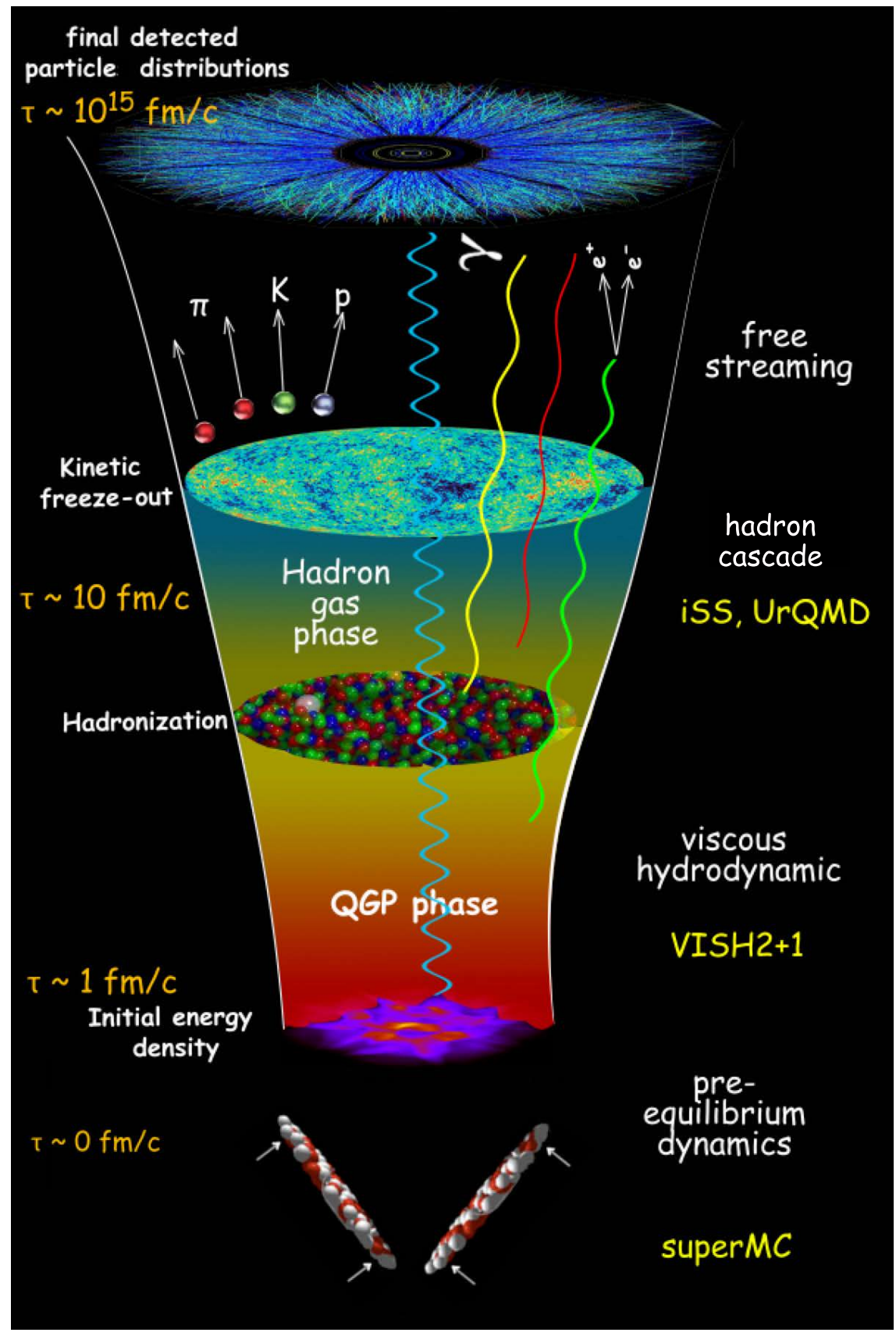

Figure 1: Illustration of the evolution of the fireball created in relativistic heavy-ion collisions, together with the theoretical model used in each stage.

during the fireball evolution. This advantage over strongly interacting probes makes them the 
cleanest penetrating probe for the heavy-ion collisions. Hadrons can only break free at the final kinetic freeze-out surface. Their measured momentum distribution carries indirect time integrated evolution information about the fireball. On the other hand, a large fraction of the thermal photons are produced early inside the fireball. Their momentum distribution preserves the dynamical information of the medium directly at their production points. Electromagnetic probes can thus provide us with constraints on the early dynamics of the fireball that are complementary to those obtained from the much more abundant hadronic observables. In Sec. 8, we will discuss the interface which coupled the event-by-event viscous hydrodynamic evolution with thermal photon radiations.

The entire integrated package is open source ${ }^{1}$ and can be freely downloaded from https: $/ /$ u.osu.edu/vishnu. Other viscous relativistic hydrodynamic codes for application to relativistic heavy-ion collisions have been developed, and results obtained with them have been reported in the literature. These include the $(2+1)$-d pure viscous hydrodynamic code $\mathrm{v}$-USPhydro [23]; the (2+1)-dimensional hybrid code SONIC [24], publicly available at https://sites. google.com/site/revihy/home, which interfaces a strongly-coupled pre-equilibrium phase based on the AdS/CFT correspondence with the (2+1)-d viscous hydrodynamic code $\mathrm{VH} 2+1$ and a hadronic cascade; the (3+1)-dimensional hybrid code IPGlasma+MUSIC+UrQMD [25] which couples a weakly-coupled pre-equilibrium stage based on classical Yang-Mills-evolution of fluctuating gluon fields [26] to $(3+1)$-dimensional viscous hydrodynamics [9, 27] and the UrQMD hadron cascade; and a number of pure viscous hydrodynamic codes in $3+1$ dimensions: the Frankfurt-Kiev code [11], the Jyväskylä-Frankfurt-Debrecen code [28, 29], the Krakow code [30, 31], the ECHO-QGP code [12], the CLVisc code [32], and the Nagoya code [33].

\section{General Framework}

Every relativistic heavy-ion collision is a multi-stage system. In our hybrid package, there is a specific code simulating each stage of the evolution. A python shell script links all the individual programs together to perform large-scale event-by-event simulations of relativistic heavy-ion collisions. The major components include the initial condition generator (superMC), a (2+1)d viscous hydrodynamic simulator (VISHNew), a particle sampler (iSS), and a hadron cascade simulator (UrQMD). In the next section, we will discuss in some detail the physics implemented in these codes.

To perform event-by-event simulations on multiple computing cores, for example using $N$ cores on a cluster, we divide the total number of events, $N_{\mathrm{ev}}$, into $N$ jobs with $M=N_{\mathrm{ev}} / N$ events in each jobs. Then we submit these $N$ jobs in parallel. The $M$ events within each job run sequentially.

\subsection{Work flow for a single sequential simulation}

For each job, the work flow is summarized in Fig. 2. The job is started by generating $M$ fluctuating initial conditions with the Monte-Carlo generator supermd2 Then each initial entropy density profile is evolved with the viscous hydrodynamic model, VISHNew [4, 14]. At the end of the hydrodynamic simulation, a switching hypersurface is identified and fluid cells

\footnotetext{
${ }^{1}$ Except for the UrQMD component, the iEBE-VISHNU package is made available under the GNU general public license v3.0.

${ }^{2}$ superMC is based on the code package mckt 34 35
} 


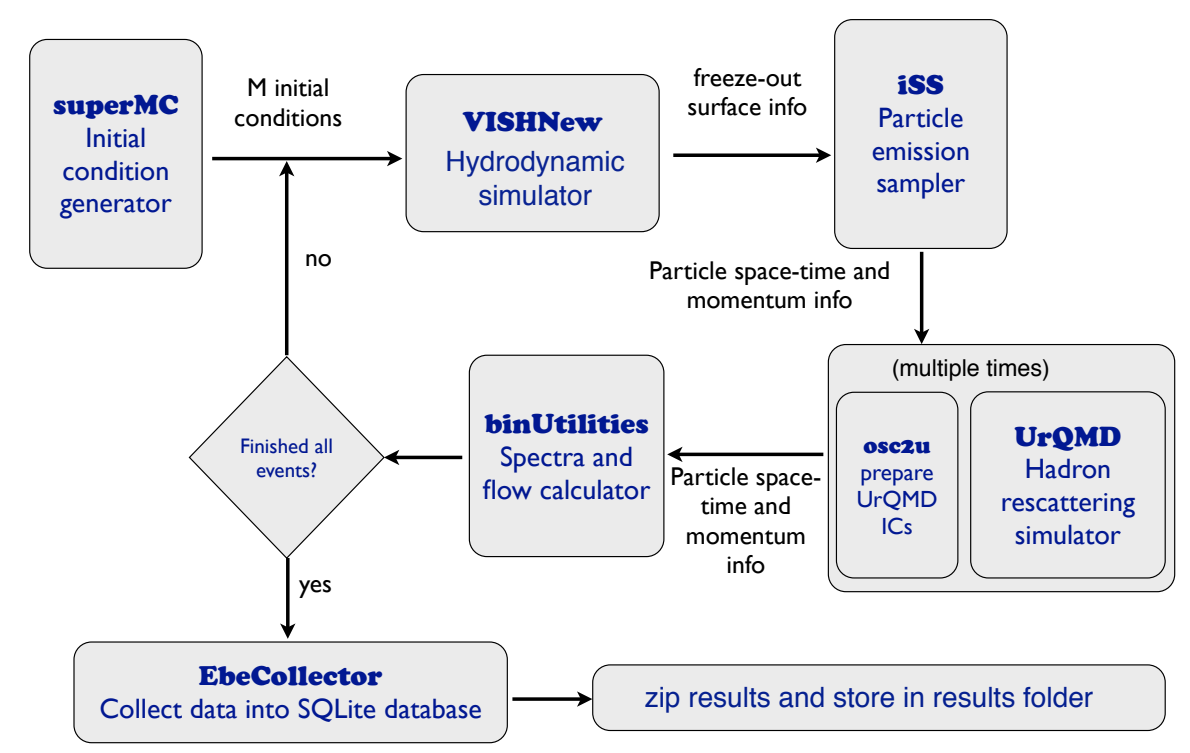

Figure 2: The work flow for a single job with $M$ events.

on this switching hyper-surface are converted into individual particles using the particle sampler, iSS. These particles are fed into UrQMD [18], a hadronic rescattering cascade which follows the particles microscopically until they stop interacting and (if unstable) decay ${ }^{3}$ The combination of the hydrodynamic evolution algorithm for the QGP stage with a microscopic hadronic cascade forms a hybrid algorithm with the name VISHNU (VISH2+1 'n' UrQMD) [36]. In the end, we collect the final particle information (momenta and positions of their last interactions or decays) from all the $M$ events using binUtilities, store the final analyzed results in the SQLite database using EbeCollector, and zip everything.

\subsection{Large scale event-by-event simulations}

For large-scale event-by-event simulations, two additional python scripts are used to generate and submit multiple jobs as illustrated in Fig. 3. Users specify the number of jobs and the number of events within each job through generateJobs.py which sets up the entire simulation and then use the script submitJobs_local.py or submitJobs_qsub.py to submit all the jobs to a local cluster or to a qsub system on the Ohio Supercomputer Center (OSC), respectively. Easy adjustments of those latter python scripts can adapt the package to other supercomputing facilities or the Open Science Grid.

After all $N$ jobs are finished, the database files from each job will be combined into one for future physics analysis of the output by users. A user friendly python package tool, UHG

\footnotetext{
${ }^{3}$ To accumulate statistics, the UrQMD casacade is optionally run multiple times (with different sampled particles from iSS) for each hydrodynamic simulation.
} 


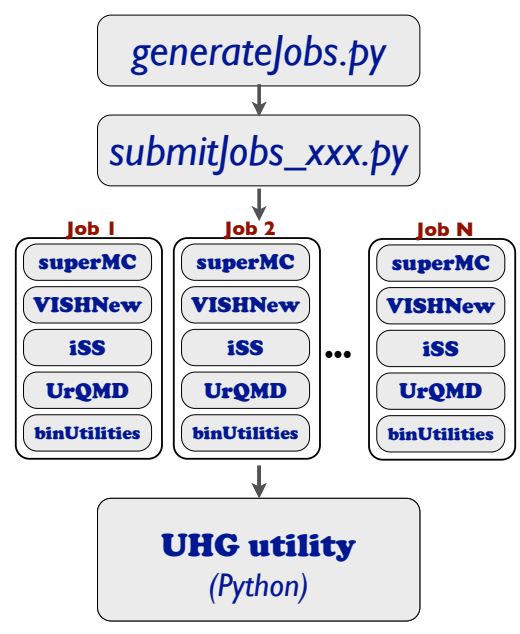

Figure 3: Work flow for multiply jobs in the large scale of event-by-event simulations.

utility, is provided for querying the database and computing experimental observables and performing various statistical analyses.

\section{Initial condition generator SuperMC}

SuperMC generates fluctuating initial conditions using the Monte-Carlo Glauber (MC-Glauber) [37-39] or Monte-Carlo Kharzeev-Levin-Nardi (MCKLN) [40, 41] models. These models can be run in several distinct modes as selected by the user.

\subsection{Collision geometry}

In relativistic heavy-ion collisions, the colliding nuclei are accelerated almost to the speed of light. Due to time dilation, nucleons' intrinsic orbital motion is frozen during the interaction period. Thus we can use a Monte Carlo procedure to sample the position of every nucleon inside the projectile and target nuclei according to their Woods-Saxon distribution.

We take into account the finite size for each individual nucleon. The density distribution of strongly interacting matter for each nucleon is given by

$$
\rho_{n}(\overrightarrow{\mathbf{r}})= \begin{cases}\frac{\theta\left(r_{1}-r_{n}\right)}{\pi r_{n}^{n}} \frac{\theta(L-\mid z))}{L}, & \text { cylindrical nucleon, } \\ \frac{1}{(2 \pi B)^{3 / 2}} e^{-r^{2} /(2 B)}, & \text { gaussian nucleon. }\end{cases}
$$

The approximation of a homogeneous cylindrical nucleon density distribution has been popular in the past since it leads to a very simple collision criterium. In this approximation, the transverse radius $r_{n}=\frac{1}{2} \sqrt{\frac{\sigma_{N N}^{\text {inel }}}{\pi}}$, where the factor of 2 accounts for the quantum mechanical nature of the nucleon-nucleon scattering process. Along the $z$ direction, $L=2 r_{n}$. A more realistic modeling takes a gaussian density distribution for the nucleon with an energy-dependent width 
$\left.B=B\left(\sqrt{s_{\mathrm{NN}}}\right)=\frac{\sigma_{\mathrm{NN}}^{\text {in }}\left(\sqrt{s_{\mathrm{NN}}}\right)}{8 \pi}[42]\right]^{4}$ The corresponding nucleon thickness functions in the transverse plane are

$$
T_{n}\left(r_{\perp}\right)=\left\{\begin{array}{cl}
\frac{\theta\left(r_{\perp}-r_{n}\right)}{\pi r_{n}^{2}}, & \text { cylindrical nucleon } \\
\frac{1}{(2 \pi B)} e^{-r_{\perp}^{2} /(2 B)}, & \text { gaussian nucleon. }
\end{array}\right.
$$

With the finite size of each nucleon, in order to reproduce the correct Woods-Saxon distribution for the density of the entire nucleus, we need to sample the nucleon positions according to a modified Woods-Saxon distribution such that, when folded with the nucleon density distribution [43-45], it reproduces the correct experimentally measured Woods-Saxon distribution:

$$
\begin{gathered}
\rho_{\mathrm{WS}}^{\exp }(\overrightarrow{\mathbf{r}})=\int d^{3} \mathbf{r}^{\prime} \tilde{\rho}_{\mathrm{WS}}\left(\overrightarrow{\mathbf{r}^{\prime}}\right) \rho_{n}\left(\overrightarrow{\mathbf{r}}-\overrightarrow{\mathbf{r}^{\prime}}\right) . \\
\tilde{\rho}_{\mathrm{WS}}(\overrightarrow{\mathbf{r}})=\frac{\rho_{0}}{1+\exp \left(\frac{r-R_{A} \Omega(\theta)}{\xi}\right)},
\end{gathered}
$$

where $\rho_{0}$ is the nucleon number density in infinite nuclear matter, $R_{A}$ is the rms charge radius of nucleus $A$, and $\xi$ is the surface width parameter. For a deformed nucleus with nonzero quadrupole and hexadecupole ground state deformation [37, 39, 43, 45] $R(\theta)=R_{A} \Omega(\theta)=$ $R_{A}\left(1+\beta_{2} Y_{0}^{2}(\theta)+\beta_{4} Y_{0}^{4}(\theta)\right)$, where $Y_{0}^{2}(\theta)$ and $Y_{0}^{4}(\theta)$ are the spherical harmonics, describes the angular dependence of the nuclear radius. In Table 2, we list the parameters used in superMC for some typical colliding nuclei.

\begin{tabular}{cc|ccccc}
\hline \hline Element & Atomic Mass & $\rho_{0}\left(\mathrm{fm}^{-3}\right)$ & $R_{A}(\mathrm{fm})$ & $\xi(\mathrm{fm})$ & $\beta_{2}$ & $\beta_{4}$ \\
\hline $\mathrm{Cu}$ & 63 & 0.1686 & 4.28 & 0.50 & 0.162 & 0.006 \\
$\mathrm{Au}$ & 197 & 0.1695 & 6.42 & 0.45 & -0.130 & -0.030 \\
$\mathrm{~Pb}$ & 208 & 0.1610 & 6.67 & 0.44 & 0 & 0 \\
$\mathrm{U}$ & 238 & 0.1660 & 6.86 & 0.44 & 0.280 & 0.093 \\
\hline \hline
\end{tabular}

Table 2: Parameters for the modified Woods-Saxon density distribution, $\tilde{\rho}_{\mathrm{Ws}}$, for some heavy nuclei that have been used in relativistic heavy-ion collisions.

Note that our parameterization of the nuclear density distribution does not account for the existence of a neutron skin in large nuclei. Inclusion of a neutron skin is left for a future improvement of the superMC code.

\subsection{The MC-Glauber approach}

The density of the inelastic cross section $\sigma_{\mathrm{NN}}^{\text {inel }}$ at impact parameter $\overrightarrow{\mathbf{b}}$ is

$$
P(\overrightarrow{\mathbf{b}})=\left\{\begin{array}{cl}
\theta\left(2 r_{n}-b\right), & \text { cylindrical nucleon, } \\
1-\exp \left(-\sigma_{\mathrm{gg}} T_{\mathrm{nn}}(b)\right), & \text { gaussian nucleon. }
\end{array}\right.
$$

\footnotetext{
${ }^{4}$ In this expression we changed the denominator from the value 14.30 suggested in [42] to $8 \pi$ because this yields a better description of the measured multiplicity distributions in $\mathrm{p}+\mathrm{Pb}$ collisions at the LHC, discussed in Sec. 3.4 This choice agrees with the naive ansatz $\sigma_{\mathrm{NN}}^{\text {inel }}=2 \pi(2 R)^{2}$, expressing the inelastic cross section as the area of a disk with radius $2 R$ where $R=\sqrt{B}$ is the rms radius of a nucleon.
} 
where $\sigma_{\mathrm{gg}}$ is the inelastic gluon-gluon cross-section [42] and $T_{\mathrm{nn}}$ is the nucleon-nucleon overlap function,

$$
T_{\mathrm{nn}}(b)=\int d^{2} \overrightarrow{\mathbf{r}}_{\perp} T_{n}\left(\overrightarrow{\mathbf{r}}_{\perp}\right) T_{n}\left(\overrightarrow{\mathbf{b}}-\overrightarrow{\mathbf{r}}_{\perp}\right)=\frac{e^{-b^{2} /(4 B)}}{4 \pi B} .
$$

For unpolarized nucleons the nucleon density is spherically symmetric, so $T_{\mathrm{nn}}(b)$ has no directional dependence. A binary collision involving nucleon pair $(i, j)$ will deposit a certain amount of energy in the medium around the collision point $\overrightarrow{\mathbf{R}}_{i j, \perp}=\frac{1}{2}\left(\overrightarrow{\mathbf{r}}_{i \perp}+\overrightarrow{\mathbf{r}}_{j \perp}\right)$. After thermalization, this energy density is associated with a corresponding amount of entropy density computable from the equation of state. For cylindrical nucleons, we choose a disk-like profile for the deposited energy or entropy density in the transverse plane. For Gaussian nucleons, the deposited energy density is modeled by a gaussian distribution. Thus, the entropy or energy density generated by all the binary collision pairs in the transverse plane is proportional to,

$$
B C\left(\overrightarrow{\mathbf{r}}_{\perp}\right)=\left\{\begin{array}{cl}
\sum_{(i, j) \in \text { pairs }} \frac{\theta\left(r_{n}-\left|\overrightarrow{\mathbf{r}}_{\perp}-\overrightarrow{\mathbf{R}}_{i j, \perp}\right|\right)}{\pi r_{n^{2}}^{2}}, & \text { cylindrical nucleons, } \\
\sum_{(i, j) \in \text { pairs }} \frac{1}{2 \pi B} e^{-\left|\overrightarrow{\mathbf{r}}_{\perp}-\mathbf{R}_{i j, \perp}\right|^{2} /(2 B)}, & \text { gaussian nucleons. }
\end{array}\right.
$$

The parameters $r_{n}$ and $B$ are chosen to be the same as in the definition of the shape of the nucleon, Eq. (1).

Every nucleon that participates in an inelastic collision is "wounded" and will "bleed" energy density into the medium. In superMC, two distinct ways to distribute the energy deposited by the wounded nucleons are implemented.

The first option is to deposit the energy azimuthally symmetrically around the center of the wounded nucleon. The total energy or entropy density contributed by all wounded nucleons is then proportional to

$$
W N\left(\overrightarrow{\mathbf{r}}_{\perp}\right)=\left\{\begin{array}{cl}
\sum_{i \in \text { wounded }} \frac{\theta\left(r_{n}-\mid \overrightarrow{\mathbf{r}}_{\perp}-\overrightarrow{\mathbf{r}}_{i \perp}\right)}{\pi r_{n}^{2}}, & \text { cylindrical nucleons, } \\
\sum_{i \in \text { wounded }} \frac{1}{2 \pi B} e^{-\left|\overrightarrow{\mathbf{r}}_{\perp}-\overrightarrow{\mathbf{r}}_{i \perp}\right|^{2} /(2 B)}, & \text { gaussian nucleons. }
\end{array}\right.
$$

where the index $i$ runs over all wounded nucleons in both nuclei A and B.

In the second approach, the energy bled from each wounded nucleon is distributed evenly over its binary collision partners and deposited azimuthally symmetrically around their correspond binary collision points. In this case, the total energy or entropy density contributed by all wounded nucleons is proportional to

$$
W N\left(\overrightarrow{\mathbf{r}}_{\perp}\right)=\left\{\begin{array}{cl}
\sum_{i \in \text { wounded }} \sum_{j=1}^{N_{b, i}} \frac{1}{N_{b, i}} \frac{\theta\left(r_{n}-\left|\overrightarrow{\mathbf{r}}_{\perp}-\overrightarrow{\mathbf{R}}_{i, \perp}\right|\right)}{\pi r_{n}^{2}}, & \text { cylindrical nucleon, } \\
\sum_{i \in \text { wounded }} \sum_{j=1}^{N_{b, i}} \frac{1}{N_{b, i}} \frac{1}{2 \pi B} e^{-\left|\overrightarrow{\mathbf{r}}_{\perp}-\overrightarrow{\mathbf{R}}_{i, \perp}\right|^{2} /(2 B)}, & \text { gaussian nucleon. }
\end{array}\right.
$$

where $N_{b, i}$ is the number of binary collision partners associated with wounded nucleon $i$. This way of distributing the energy density is motivated by the idea that the inelastic collisions between nucleons that generate wounded nucleons or binary collision events are fundamentally the same.

The second approach distributes the entropy or energy density of wounded nucleons over a more compact transverse area, which in the end result increases the initial eccentricity of the fireball created in the collision at large impact parameters. In central collisions, the difference in eccentricity between the two energy deposition schemes is negligible. 
In the MC-Glauber model, the total energy density produced in the transverse plane after thermalization is taken to be a mixture of the wounded nucleon and binary collision density profiles [46, 47]:

$$
\left\{\begin{array}{c}
s_{0}\left(\overrightarrow{\mathbf{r}}_{\perp}\right) \\
e_{0}\left(\overrightarrow{\mathbf{r}}_{\perp}\right)
\end{array}\right\}=\frac{1}{\tau_{0}}\left\{\begin{array}{c}
\kappa_{s} \\
\kappa_{e}
\end{array}\right\}\left(\frac{1-\alpha}{2} W N\left(\overrightarrow{\mathbf{r}}_{\perp}\right)+\alpha B C\left(\overrightarrow{\mathbf{r}}_{\perp}\right)\right),
$$

Here $\alpha$ is the binary mixing parameter and $\kappa$ is an overall normalization factor $\kappa$ is tuned to reproduce to measured final charged multiplicity in the most central collisions, while $\alpha$ is adjusted to reproduce its observed dependence on collision centrality. Due to viscous heating during the hydrodynamic expansion, the normalization $\kappa$ depends on the specific shear viscosity $\eta / s$. In Table 3, we list the values of $\kappa_{s}$ for several values of $\eta / s$ at RHIC and LHC energies.

\begin{tabular}{c|c|c|c}
\hline \hline & $\mathrm{Au}+\mathrm{Au} @ 200 \mathrm{~A} \mathrm{GeV}$ & $\mathrm{Pb}+\mathrm{Pb} \mathrm{@} \mathrm{2.76} \mathrm{A} \mathrm{TeV}$ & $\mathrm{Pb}+\mathrm{Pb} \mathrm{@} \mathrm{5.5} \mathrm{A} \mathrm{TeV}$ \\
\hline$\eta / s=0.08$ & 17.900 & 34.591 & 40.132 \\
$\eta / s=0.12$ & 16.694 & 32.759 & 38.161 \\
$\eta / s=0.16$ & 15.492 & 30.908 & 36.148 \\
$\eta / s=0.20$ & 14.290 & 29.040 & 34.116 \\
\hline
\end{tabular}

Table 3: The normalization factor $\kappa_{s}$ for the different values of $\eta / s$ at the RHIC and LHC energies for MC-Glauber model.

\subsection{The MCKLN approach}

The MCKLN model [40, 41] is based on a $k_{T}$-factorization ansatz [40, 41] in which the produced gluon density distribution can be calculated as

$$
\begin{aligned}
\frac{d N_{g}}{d y d^{2} p_{\perp} d^{2} x_{\perp}} & =\frac{2 \pi^{3} N_{c}}{N_{c}^{2}-1} \int_{0}^{p_{\perp}} d^{2} k_{\perp} \frac{\alpha_{s}\left(\max \left\{\left(\left(\overrightarrow{\mathbf{p}}_{\perp}+\overrightarrow{\mathbf{k}}_{\perp}\right) / 2\right)^{2},\left(\left(\overrightarrow{\mathbf{p}}_{\perp}-\overrightarrow{\mathbf{k}}_{\perp}\right) / 2\right)^{2}\right\}\right)}{p_{\perp}^{2}} \\
& \times \phi_{A}\left(x_{1},\left(\frac{\overrightarrow{\mathbf{p}}_{\perp}+\overrightarrow{\mathbf{k}}_{\perp}}{2}\right)^{2} ; \overrightarrow{\mathbf{x}}_{\perp}+\overrightarrow{\mathbf{b}} / 2\right) \\
& \times \phi_{B}\left(x_{2},\left(\frac{\overrightarrow{\mathbf{p}}_{\perp}-\overrightarrow{\mathbf{k}}_{\perp}}{2}\right)^{2} ; \overrightarrow{\mathbf{x}}_{\perp}-\overrightarrow{\mathbf{b}} / 2\right),
\end{aligned}
$$

where $\alpha_{s}$ is the strong coupling constant and $\phi_{A}$ and $\phi_{B}$ are the unintegrated gluon distribution functions of the two colliding nucleus. $\overrightarrow{\mathbf{p}}_{\perp}=\frac{\overrightarrow{\mathbf{p}}_{1 \perp}+\overrightarrow{\mathbf{p}}_{2 \perp}}{2}$ and $\overrightarrow{\mathbf{k}}_{\perp}=\overrightarrow{\mathbf{p}}_{1 \perp}-\overrightarrow{\mathbf{p}}_{2 \perp}$, where $\overrightarrow{\mathbf{p}}_{1(2) \perp}$ are the transverse momenta of the fusing gluons from the two nuclei and $x_{1(2)}=\frac{p_{\perp}}{\sqrt{s_{\mathrm{N}}}} e^{ \pm y}$ are their corresponding light-cone momentum fractions. The unintegrated gluon distribution function is parameterized as,

$$
\phi\left(x, k^{2} ; \overrightarrow{\mathbf{x}}_{\perp}\right)=\kappa \frac{N_{c}^{2}-1}{2 N_{c}} \frac{Q_{s}^{2}\left(x, \overrightarrow{\mathbf{x}}_{\perp}\right)}{2 \pi^{3} \alpha_{s}\left(Q_{s}^{2}\right)}\left\{\begin{array}{ll}
\frac{1}{Q_{s}^{2}+\Lambda^{2}}, & k \leq Q_{s} \\
\frac{1}{k^{2}+\Lambda^{2}}, & k>Q_{s}
\end{array},\right.
$$

where $\Lambda=\Lambda_{\mathrm{QCD}}=0.2 \mathrm{GeV}$, and $\kappa=1.8$ is a phenomenological parameter adjusted [48] to fit the measured charged multiplicity at mid rapidity in the most central $\mathrm{Au}+\mathrm{Au}$ collisions at $\sqrt{s_{\mathrm{NN}}}=200 \mathrm{GeV}$ at RHIC. The saturation scale is given by the implicit relation,

$$
Q_{s}^{2}\left(x, \overrightarrow{\mathbf{x}}_{\perp}\right)=\frac{4 \pi^{2} N_{c}}{N_{c}^{2}-1} \alpha_{s}\left(Q_{s}^{2}\right) x G\left(x, Q_{s}^{2}\right) T_{A}\left(\overrightarrow{\mathbf{x}}_{\perp}\right)
$$


The running coupling strength is parameterized as,

$$
\alpha_{s}\left(k^{2}\right)=\left\{\begin{array}{cc}
\frac{4 \pi}{\beta_{0} \ln \left(\left(k^{2}+\Lambda^{2}\right) / \Lambda_{\mathrm{QCD}}^{2}\right)}, & \alpha_{s} \leq 0.5 \\
0.5, & \alpha_{s} \geq 0.5
\end{array},\right.
$$

with $\beta_{0}=11-\frac{2}{3} N_{f}$. Kharzeev, Levin, and Nardi [41] use the parameterization $x G\left(x, k^{2}\right)=$ $K \ln \left(\frac{k^{2}+\Lambda^{2}}{\Lambda_{\mathrm{QCD}}^{2}} x^{-\lambda}(1-x)^{4}\right.$ with $\lambda=0.2$ and $K=0.7$ adjusted such that the average $Q_{s}^{2}$ in the transverse plane of a central $200 \mathrm{~A} \mathrm{GeV} \mathrm{Au}+\mathrm{Au}$ collision, $\left\langle Q_{s}^{2}(x=0.01)\right\rangle \simeq 2.0 \mathrm{GeV}^{2}$. [48] Inserting this into Eq. 13 and dropping the $(1-x)^{4}$ factor since $x$ is small in the kinematic region of interest leads to

$$
Q_{s}^{2}\left(x, \overrightarrow{\mathbf{x}}_{\perp}\right)=2 \mathrm{GeV}^{2}\left(\frac{T\left(\overrightarrow{\mathbf{x}}_{\perp}\right)}{T_{0}}\right)\left(\frac{x_{0}}{x}\right)^{\lambda}
$$

where $T_{0}=1.53 \mathrm{fm}^{-2}$ and $x_{0}=0.01$ [49].

The initial entropy density in the transverse is assumed to be proportional to the $p_{T}$-integrated produced gluon density,

$$
s\left(\overrightarrow{\mathbf{x}}_{\perp}\right)=\frac{\kappa}{\tau_{0}} \int d^{2} p_{\perp} \frac{d N_{g}}{d y d^{2} p_{\perp} d^{2} x_{\perp}} .
$$

Table 4 lists the values of the normalization factor $\kappa$ for different $\eta / s$, which are fixed to reproduce the top $0-5 \%$ final charged hadron multiplicity at the mid-rapidity.

\begin{tabular}{l|c|c|c}
\hline \hline & $\mathrm{Au}+\mathrm{Au} @ 200 \mathrm{~A} \mathrm{GeV}$ & $\mathrm{Pb}+\mathrm{Pb} \mathrm{@} \mathrm{2.76} \mathrm{A} \mathrm{TeV}$ & $\mathrm{Pb}+\mathrm{Pb} \mathrm{@} \mathrm{5.5} \mathrm{A} \mathrm{TeV}$ \\
\hline$\eta / s=0.08$ & 5.692 & 6.998 & 7.628 \\
$\eta / s=0.12$ & 5.309 & 6.625 & 7.250 \\
$\eta / s=0.16$ & 4.923 & 6.255 & 6.871 \\
$\eta / s=0.20$ & 4.541 & 5.878 & 6.486 \\
\hline \hline
\end{tabular}

Table 4: The normalization factor $\kappa$ for the different values of $\eta / s$ at the RHIC and LHC energies for MC-KLN model.

\subsection{Collision-by-collision multiplicity fluctuations}

The entropy (or energy) density dumped into the medium from each binary collision and wounded nucleon can fluctuate. These fluctuations lead to the measured multiplicity fluctuation in pp collisions. We denote such fluctuation as collision-by-collision multiplicity fluctuations.

In 2012, the CMS collaboration measured flow observables in $0-0.2 \%$ ultra-central $\mathrm{Pb}+\mathrm{Pb}$ collisions at the LHC [50]. For these extremely high multiplicity and extremely rare heavy-ion collision events, the event selection is strongly biased towards upward fluctuations in the particles production of the system. Thus, we would expect collision-by-collision multiplicity fluctuations to become important for the event selection in such ultra-central collisions.

In superMC, we implement collision-by-collision multiplicity fluctuations in the MC-Glauber model based on the phenomenological KNO scaling observed in pp collisions [51, 52]. In the MC-Glauber model, we regard each binary collision and each wounded nucleon as an independent source of energy with stochastic norm. This can be expressed through the following modification of Eq. (7) and (8):

$$
B C\left(\overrightarrow{\mathbf{r}}_{\perp}\right)=\left\{\begin{array}{cl}
\sum_{(i, j) \in \text { pairs }} \gamma_{i, j} \frac{\theta\left(r_{n}-\left|\overrightarrow{\mathbf{r}}_{\perp}-\overrightarrow{\mathbf{R}}_{i j, \perp}\right|\right)}{\pi r_{n}^{2}}, & \text { cylindrical nucleons, } \\
\sum_{(i, j) \in \text { pairs }} \gamma_{i, j} \frac{1}{2 \pi B} e^{-\left|\overrightarrow{\mathbf{r}}_{\perp}-\overrightarrow{\mathbf{R}}_{i j, \perp}\right|^{2} /(2 B)}, & \text { gaussian nucleon. }
\end{array}\right.
$$


and

$$
W N\left(\overrightarrow{\mathbf{r}}_{\perp}\right)=\left\{\begin{array}{cl}
\sum_{i \in \text { wounded }} \gamma_{i} \frac{\theta\left(r_{n}-\left|\overrightarrow{\mathbf{r}}_{\perp}-\overrightarrow{\mathbf{r}}_{i \perp}\right|\right)}{\pi r_{n}^{2}}, & \text { cylindrical nucleon, } \\
\sum_{i \in \text { wounded }} \gamma_{i} \frac{1}{2 \pi B} e^{-\left|\overrightarrow{\mathbf{r}}_{\perp} \overrightarrow{\mathbf{r}}_{i \perp}\right|^{2} /(2 B)}, & \text { gaussian nucleon. }
\end{array}\right.
$$

where the multiplicity scaling factors $\gamma_{i, j}$ and $\gamma_{i}$ are continuous random variables with unit mean values. In practice, we use the Gamma distribution as the probability distribution for $\gamma_{i, j}$ and $\gamma_{i}$. The Gamma distribution for a random variable $X$ is defined as

$$
\operatorname{Gamma}(X)=\frac{1}{\Gamma(k) \theta^{k}} x^{k-1} e^{-x / \theta},
$$

where $k$ and $\theta$ are the so-called shape and scale parameters of the Gamma distribution, respectively. The Gamma distribution is positive semi-definite and has the following properties:

(1) If $X_{i}=\operatorname{Gamma}\left(k_{i}, \theta\right)$, then $\sum_{i} X_{i}=\operatorname{Gamma}\left(\sum_{i} k_{i}, \theta\right)$.

(2) If $X=\operatorname{Gamma}(k, \theta)$, then $c X=\operatorname{Gamma}(k, c \theta)$ for any $c>0$.

By using these two properties of the Gamma distribution, we can assign two different sets of $(k, \theta)$ parameters for $\gamma_{i}$ and $\gamma_{i, j}$ in Eqs. (17) and Eqs. (18), respectively, to ensure that the final total entropy or energy density, which is a weighted sum of all the collisions in the event according to Eq. 10] also fluctuates according a Gamma distribution with a desired shape and scale. For $W N\left(\overrightarrow{\mathbf{r}}_{\perp}\right)$, we write,

$$
\gamma_{i}=\operatorname{Gamma}\left(k_{W N}, \theta_{W N}\right)
$$

and for $B C\left(\overrightarrow{\mathbf{r}}_{\perp}\right)$

$$
\gamma_{i, j}=\operatorname{Gamma}\left(k_{B C}, \theta_{B C}\right) .
$$

Based on Eqs. (10), 17) and (18), we then have

$$
s=\kappa\left(\operatorname{Gamma}\left(\sum_{i=1}^{N_{\text {part }}} k_{W N, i}, \frac{1-\alpha}{2} \theta_{W N}\right)+\operatorname{Gamma}\left(\sum_{i=1}^{N_{\text {coll }}} k_{B C, i}, \alpha \theta_{B C}\right)\right) .
$$

By requiring $\frac{1-\alpha}{2} \theta_{W N}=\alpha \theta_{B C}=\theta$, Eq. 22 can be further simplified to,

$$
\left\{\begin{array}{c}
s \\
e
\end{array}\right\}=\left\{\begin{array}{c}
\kappa_{s} \\
\kappa_{e}
\end{array}\right\}\left(\operatorname{Gamma}\left(N_{\mathrm{part}} k_{W N}+N_{\mathrm{coll}} k_{B C}, \theta\right)\right) .
$$

By further writing $k_{W N}=\frac{1-\alpha}{2} k$ and $k_{B C}=\alpha k$, we finally obtain,

$$
\left\{\begin{array}{c}
s \\
e
\end{array}\right\}=\frac{1}{\tau_{0}}\left\{\begin{array}{c}
\kappa_{s} \\
\kappa_{e}
\end{array}\right\}\left(\operatorname{Gamma}\left(\left(\frac{1-\alpha}{2} N_{\text {part }}+\alpha N_{\text {coll }}\right) k, \theta\right)\right) .
$$

Choosing the upper variant gives for the mean entropy density $\langle s\rangle=\frac{\kappa_{s}}{\tau_{0}}\left(\frac{1-\alpha}{2} N_{\text {part }}+\alpha N_{\text {coll }}\right) k \theta$, and similarly for the mean energy density if the lower variant is chosen. Setting $k \theta=1$ ensures that with the perviously adjusted normalizations $\kappa_{s}$ or $\kappa_{e}$ the event-averaged total entropy continues to reproduce the value from the conventional MC-Glauber model (and thus the observed final charged multiplicity).

The actual value of $\theta$ with $(k=1 / \theta)$ in Eq. (24) can be fit to the multiplicity distribution measured in pp collisions (in which $N_{\text {part }}=2$ and $N_{\text {coll }}=1$ ), after folding the initial-state fluctuations with an additional Poisson distribution describing the multiplicity fluctuations generated 
by the hadronization process 5 At LHC energies, the multiplicity fluctuations in pp collision have been measured at $\sqrt{s}=0.9,2.36$, and $7 \mathrm{TeV}$ [51]. Additionally, the UA5 Collaboration measured pp multiplicity distributions at $\sqrt{s}=200 \mathrm{GeV}$ [52]. According to the KNO scaling hypothesis, $\left\langle N_{\mathrm{ch}}\right\rangle P\left(N_{\mathrm{ch}}\right)$ should be a universal (energy independent) function of the normalized multiplicity $N_{\mathrm{ch}} /\left\langle N_{\mathrm{ch}}\right\rangle$ as shown in Fig. 4 Because the mean $d N^{\mathrm{ch}} / d \eta$ in minimum bias pp collisions depends on $\sqrt{s}$, the variance of the Poisson distribution differs from one collision energy to another. Thus, the $\theta$ parameter in the Gamma distribution also depends on $\sqrt{s}$. In Table 5, we list the appropriate choice of the $\theta$ parameter at several collision energies. Our minimum- $\chi^{2}$-fit at $\sqrt{s}=5.02 \mathrm{TeV}$ is shown in Fig. 4

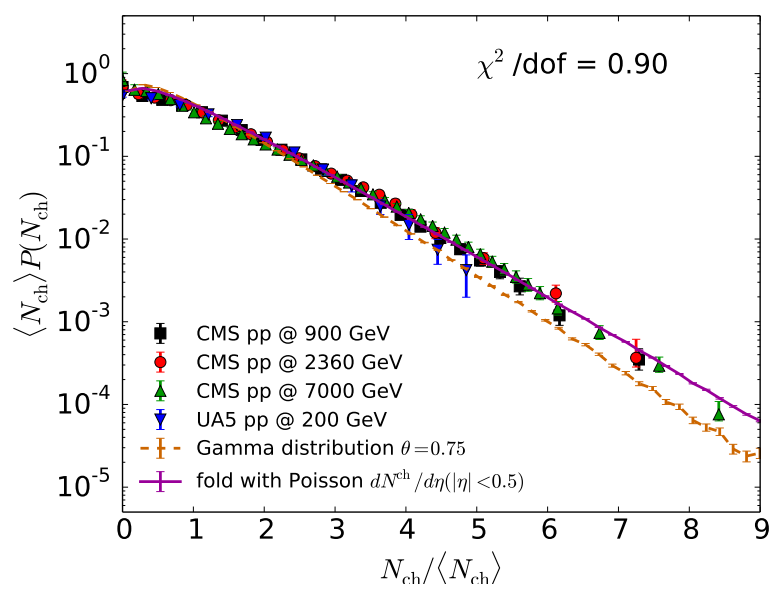

Figure 4: Normalized charged hadron multiplicity distribution for minimum bias pp collisions at $\sqrt{s}=5.02 \mathrm{TeV}$. The dashed line shows the result from a Gamma distribution with $\theta=0.75$, the solid line the distribution obtained by folding this Gamma distribution with a Poisson distribution whose mean was adjusted to the measured mean charged hadron multiplicity at this collision energy. The solid line is compared with experimental data [51,52] showing the KNO scaling of the pp multiplicity distribution in $|\eta|<0.5$ for $\sqrt{s}=200,900,2360$, and $7000 \mathrm{~A} \mathrm{GeV}$.

\begin{tabular}{c|c|c|c}
\hline \hline collision energy & $d N^{\text {ch }} /\left.d \eta\right|_{|\eta|<0.5}$ & $\theta$ & $\chi^{2} /$ d.o.f \\
\hline pp @200 GeV & 2.47 & 0.61 & 2.02 \\
pp @ $2760 \mathrm{GeV}$ & 4.54 & 0.73 & 0.96 \\
pp@ @ 5020 GeV & 5.28 & 0.75 & 0.90 \\
\hline \hline
\end{tabular}

Table 5: The choice of the $\theta$ parameter in the Gamma-distribution at several collision energies.

Once the parameters of the Gamma distribution are fixed by the phenomenological KNO scaling, we use this model to make a parameter-free postdiction for the multiplicity distributions in $\mathrm{p}+\mathrm{Pb}$ collisions at $\sqrt{s_{\mathrm{NN}}}=5.02 \mathrm{TeV}$. In the MC-Glauber model, collision-by-collision multiplicity fluctuations significantly increase the probability for upward fluctuations in the multiplicity for $\mathrm{p}+\mathrm{Pb}$ collisions. The MC-KLN model (which does not account for pp multiplicity fluctuations) produces the narrowest distribution for the initial total entropy at mid-rapidity. In

\footnotetext{
${ }^{5}$ A Gamma distribution folded with a Poisson distribution results in a negative binomial distribution.
} 

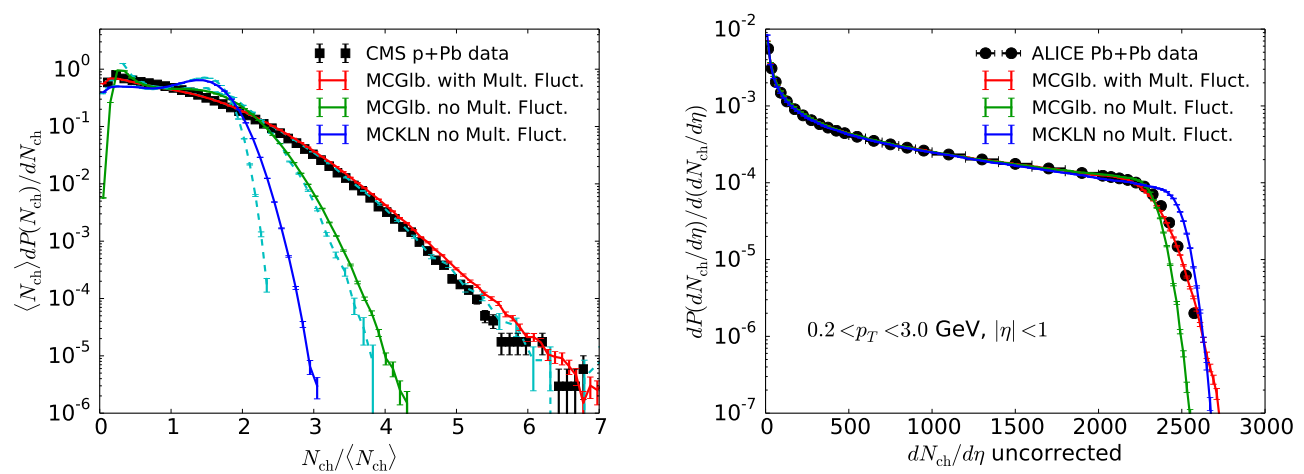

Figure 5: Left panel: Normalized multiplicity distributions for the MC-Glauber and the MCKLN models in $\mathrm{p}+\mathrm{Pb}$ collisions at $\sqrt{s_{\mathrm{NN}}}=5.02 \mathrm{TeV}$ compared with the CMS measurements [53]. The normalized distribution of the initial entropy density, $d S / d y$ are shown as the dashed cyan lines. Right panel: Comparisons of charged hadron multiplicity distribution in $\mathrm{Pb}+\mathrm{Pb}$ collisions at $\sqrt{s_{\mathrm{NN}}}=2.76 \mathrm{TeV}$ with ALICE measurement [54].

order to compare with the $\mathrm{p}+\mathrm{Pb}$ multiplicity distribution measured by $\mathrm{CMS}$ we first convert the initial total entropy to final charged hadron multiplicity, assuming they are proportional to each other. Choosing the same kinematic cuts as used in the CMS measurement, $p_{T}>0.4 \mathrm{GeV}$ and $|\eta|<2.4$ [53], we map $d N^{\text {ch }} / d \eta \simeq \frac{4.8 \times 0.75}{8.9} d S /\left.d y\right|_{y=0}$. The mean charged hadron multiplicity in minimum bias $\mathrm{p}+\mathrm{Pb}$ collisions obtained by this mapping lies within the measured value $50 \pm 2$ [53]. We then fold the distribution of $d N^{\text {ch }} /\left.d \eta\right|_{\left\{p_{T}>0.4 \mathrm{GeV},|\eta|<2.4\right\}}$ values calculated from the initial entropy density distribution with a Poisson distribution of multiplicity fluctuations produced at hadronization (here taken to be controlled by the mean multiplicity at kinetic freeze-out). By oversampling 20 Poisson distributions from each event, we obtained 20 million samples for each set of initial conditions. Their normalized distributions are compared with the CMS measurement in left panel of Fig. 5. The MC-Glauber model with collision-by-collision multiplicity fluctuations can reproduce the shape of the measured $\mathrm{p}+\mathrm{Pb}$ multiplicity distributions [55]. By comparing with the distribution of initial total entropy values we find that the broadening of the multiplicity distribution due to final state Poisson fluctuations becomes less important as $d N^{\text {ch }} / d \eta$ increases from $\mathrm{pp}$ collisions to $\mathrm{pPb}$ collisions. This is because the normalized variance of a Poisson distribution with mean $\lambda$ decreases as $1 / \sqrt{\lambda}$. In the right panel of Fig. 5 , we further compute the charged hadron multiplicity distribution in $\mathrm{Pb}+\mathrm{Pb}$ collisions at $\sqrt{s_{\mathrm{NN}}}=2.76 \mathrm{TeV}$. The MCGlauber model with collision-by-collision fluctuations can reproduce the ALICE measurements [54] very well.

In Fig. 6 we show a comparison of the initial spatial eccentricity $\varepsilon_{n}\{2\}$ as a function of the harmonic order $n$ for $0-0.2 \%$ ultra-central $\mathrm{Pb}+\mathrm{Pb}$ collisions at LHC energy. $\varepsilon_{n}\{2\}=\sqrt{\left\langle\varepsilon_{n}^{2}\right\rangle}$ is the rms of $\varepsilon_{n}$, defined in terms of the fluctuating initial energy density profile $e\left(r_{\perp}, \phi\right)$ as

$$
\varepsilon_{1} e^{i \Phi_{1}}=-\frac{\int d^{2} \mathbf{r}_{\perp} r_{\perp}^{3} e\left(r_{\perp}, \phi\right) e^{i \phi}}{\int d^{2} \mathbf{r}_{\perp} r_{\perp}^{3} e\left(r_{\perp}, \phi\right)}
$$

and

$$
\varepsilon_{n} e^{i n \Phi_{n}}=-\frac{\int d^{2} \mathbf{r}_{\perp} r_{\perp}^{n} e\left(r_{\perp}, \phi\right) e^{i n \phi}}{\int d^{2} \mathbf{r}_{\perp} r_{\perp}^{n} e\left(r_{\perp}, \phi\right)}, \quad \text { for } \quad n \geq 2
$$



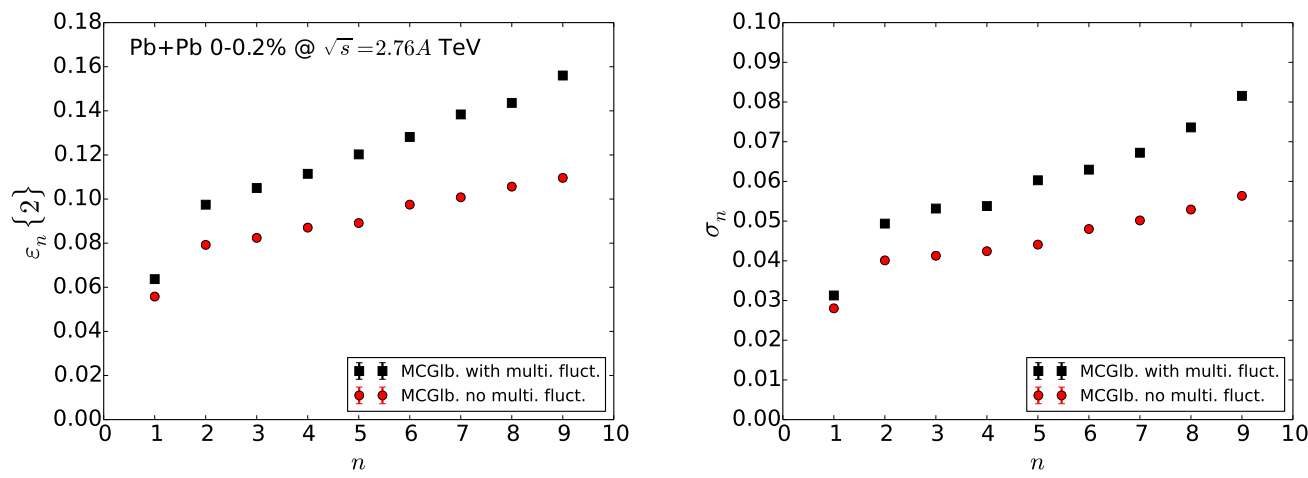

Figure 6: Left Panel: Root mean square of the $n$-th order initial spatial eccentricity as a function of the harmonic order $n$. Right Panel: The variance of $\varepsilon_{n}$ as a function of $n$. A repulsive hard core with minimum inter-nucleon distance $r_{\min }=0.9$ $\mathrm{fm}$ is imposed when sampling nucleon spatial configuration inside the nucleus.

We can clearly see that the collision-by-collision multiplicity fluctuations increase the eccentricity coefficients for all harmonic orders by $20-40 \%$. The increase is larger for higher order $n$. The multiplicity fluctuations also increase the variance of $\varepsilon_{n}$ similar amount. The existence of such fluctuations therefore changes the mean values and their variances of the initial fluctuation spectrum of the MC-Glauber model dramatically.
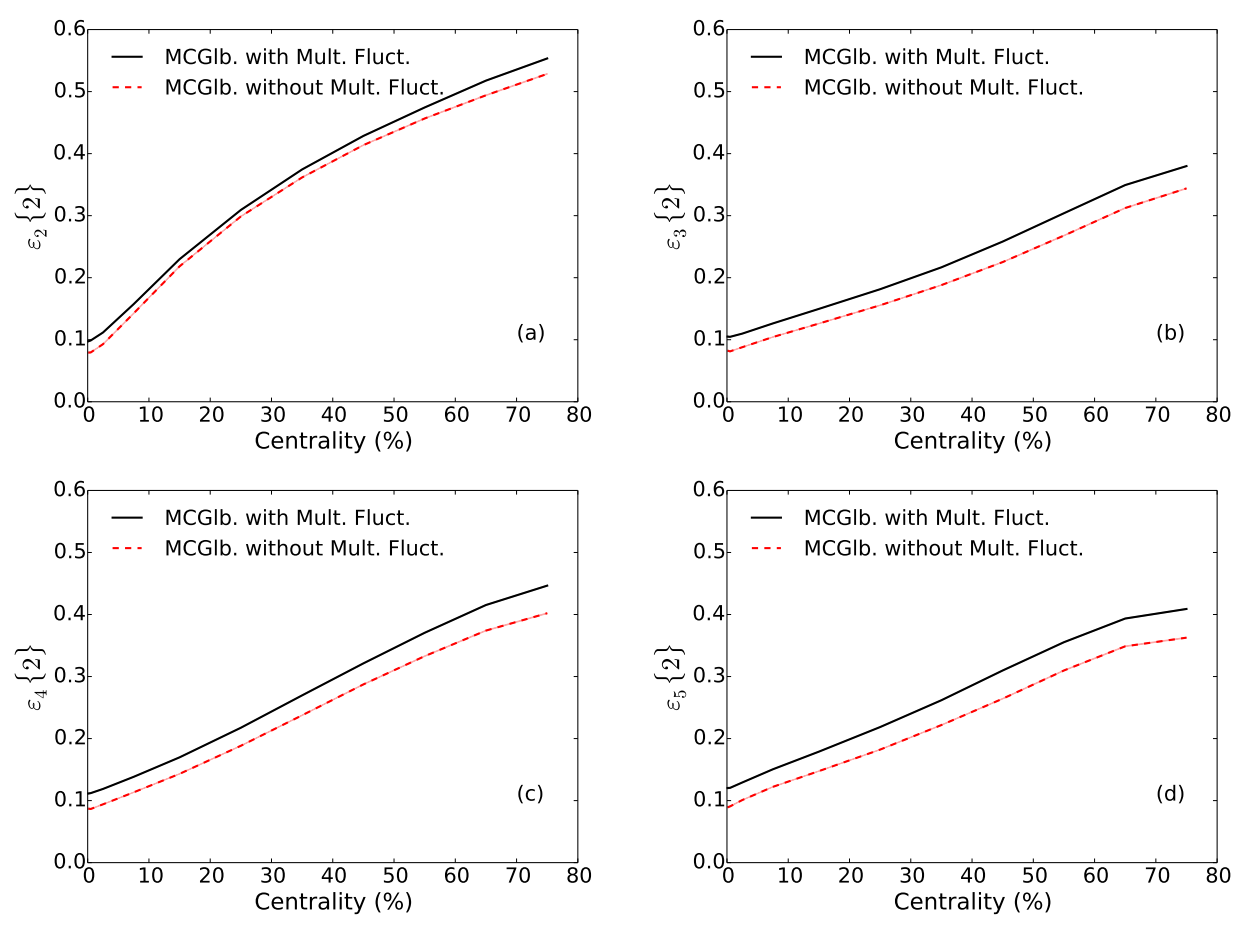

Figure 7: Centrality dependence of the root mean square initial spatial eccentricities $\varepsilon_{2,3,4,5}\{2\}$. 
In Fig. 7. we show $\varepsilon_{2}$ to $\varepsilon_{5}$ as functions of the collision centrality. We find that collision-bycollision multiplicity fluctuations are not only important in ultra-central collisions, but that they increase the spatial eccentricities at all collision centralities.

\subsection{Centrality cuts in theoretical calculations}

Centrality is a key quantity that links the theoretical calculations with the experimental measurements. It is introduced to characterize the collision geometry in nucleus-nucleus collisions. Experimentally, the centrality is typically defined by sorting the recorded events according to their measured charged hadron multiplicity at mid-rapidity, $d N^{\mathrm{ch}} /\left.d \eta\right|_{|\eta|<0.5}$. Applying the same procedure theoretically is computationally expensive since, due to viscous heating, the final charged hadron multiplicity can not be determined directly from the initially produced entropy, but requires the calculation of the full viscous hydrodynamic evolution, event by event.

However, we can use the following approximation to save simulation time: we select centrality on the initially produced total entropy in the transverse plane, $d S /\left.d y\right|_{y=0}$, assuming that, on average, the final charged hadron multiplicity, $d N^{\mathrm{ch}} / d \eta$, is monotonically related to $d S /\left.d y\right|_{y=0}$. This procedure is illustrated in Fig. 8
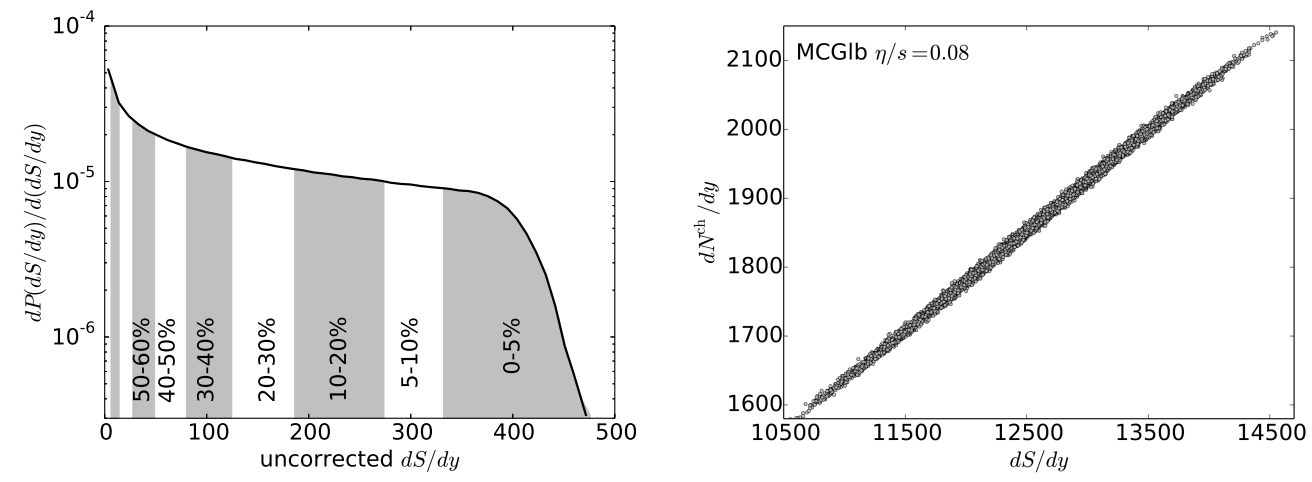

Figure 8: Left Panel: Probability distribution of the total entropy density $d S /\left.d y\right|_{y=0}$ from MC-Glauber model for Pb + $\mathrm{Pb}$ at $\sqrt{s}=2.76 A \mathrm{TeV}$. Right Panel: Correlation between initial $d S / d y$ and final measured $d N^{\mathrm{ch}} / d y$ at $0-5 \%$ most central collisions for MC-Glauber with $\eta / s=0.08$.

After having fixed the normalization constant $\kappa$ in Eq. (10) such that, on average, the measured charged multiplicity in central collisions is correctly reproduced, we first sort the minimum bias events generated by the MC-Glauber model according to the initial entropy $d S / d y$. Then, we can classify their collision centrality through their relative positions in the sorted array. The events with largest total entropy define the most central collisions where the two nuclei completely overlap each other. The $0-10 \%$ centrality bin includes the $10 \%$ of all events with the largest initial $d S / d y, 90-100 \%$ centrality the $10 \%$ of all events with the smallest $d S / d y$ values.

This procedure ignores event-by-event fluctuations in the fractional increase of the entropy due to viscous heating during the hydrodynamic evolution. This extra entropy production depends on the actual shape of the initial density profile as well as on the chosen value for the specific shear viscosity, $\eta / s$. Event-by-event fluctuation of the viscous entropy production will de-correlate the one-to-one correspondence between the initial total entropy, $d S / d y$ and the final measured charged hadron multiplicity, $d N^{\mathrm{ch}} / d y$. However, as shown in the right panel of the Fig. 8. this decorrelation is weak: For given $d S / d y$, the spread in final $d N_{\mathrm{ch}} / d y$ is very small (2-3\%). 
Therefore, our procedure of defining collision centrality by cutting on the initial total $d S / d y$ is a pretty good theoretical approximation to the experimental centrality definition using final state charged hadron multiplicities.

\section{4. (2+1)-d viscous hydrodynamics VISHNew}

\subsection{Solving the hydrodynamic equations}

The module VISHNew is an improved version of VISH2+1, the $(2+1)$-d longitudinally boostinvariant viscous hydrodynamic algorithm developed by H. Song [4, 14, 56]. It includes several improvements for efficiency and stability which will be discussed in this section. We solve the following equation of motion for second order viscous hydrodynamics ("Israel-Stewart equations"):

$$
d_{\mu} T^{\mu \nu}=0, T^{\mu \nu}=e u^{\mu} u^{v}-(p+\Pi) \Delta^{\mu \nu}+\pi^{\mu \nu} .
$$

The shear stress tensor $\pi^{\mu v}$ and bulk pressure $\Pi$ satisfy the following transport equations,

$$
\begin{aligned}
\Delta^{\mu \alpha} \Delta^{\nu \beta} D \pi_{\alpha \beta} & =-\frac{1}{\tau_{\pi}}\left(\pi^{\mu \nu}-2 \eta \sigma^{\mu \nu}\right)-\frac{1}{2} \pi^{\mu \nu} \frac{\eta T}{\tau_{\pi}} d_{\lambda}\left(\frac{\tau_{\pi}}{\eta T} u^{\lambda}\right), \\
D \Pi & =-\frac{1}{\tau_{\Pi}}(\Pi+\zeta \theta)-\frac{1}{2} \Pi \frac{\zeta T}{\tau_{\Pi}} d_{\lambda}\left(\frac{\tau_{\Pi}}{\zeta T} u^{\lambda}\right),
\end{aligned}
$$

where $D=u^{\mu} d_{\mu}$. The hydrodynamic equations need to be solved together with a given equation of state (EOS). VISHNew supports three versions of the lattice-based equation of state, s95p-v0-PCE, s95p-v1, and s95p-v1-PCE [15]. The differences among these three EOS are different implementations of partial chemical equilibrium in the hadronic phase [15, 57]. In general, Eq. 27) must be supplemented by an evolution equation (conservation law) for the baryon current $j^{\mu}=n u^{\mu}$. We start with the case $n=0$.

\subsubsection{Without baryon current}

The hydrodynamic code evolves the components of energy stress tensor. In order to use the EOS for determining the pressure in the liquid, we first need to solve for the local energy density and velocity of the fluid cell. In the (2+1)-d case, we define a vector $M^{\mu}=\left(M^{0}, M^{x}, M^{y}\right)=$ $\left(T^{\tau \tau}-\pi^{\tau \tau}, T^{\tau x}-\pi^{\tau x}, T^{\tau y}-\pi^{\tau y}\right)$. Using the decomposition Eq. 27) for $T^{\mu v}$, we find

$$
\begin{gathered}
M^{0}=(e+P+\Pi)\left(u^{0}\right)^{2}-P-\Pi, \\
M^{1}=(e+P+\Pi) u^{0} u^{1}, \\
M^{2}=(e+P+\Pi) u^{0} u^{2},
\end{gathered}
$$

The local energy density thus satisfies the following equation:

$$
e=M^{0}-\frac{\left(M^{1}\right)^{2}+\left(M^{2}\right)^{2}}{M^{0}+P+\Pi} .
$$

To solve Eq. 33 we define

$$
f(e)=\left(M^{0}-e\right)\left(M^{0}+P+\Pi\right)-\left(\left(M^{1}\right)^{2}+\left(M^{2}\right)^{2}\right) .
$$


We first observe that $f\left(M^{0}\right)=-\left(\left(M^{1}\right)^{2}+\left(M^{2}\right)^{2}\right) \leq 0$. In order for Eq. 33 to have an odd number of positive solutions, we need to require $f(0) \geqslant 0$. With a non-zero bulk viscous pressure, this leads to the condition,

$$
f(0)=\left(M^{0}\right)^{2}-\left(M^{1}\right)^{2}-\left(M^{2}\right)^{2}+M^{0} \Pi \geqslant 0 .
$$

When this requirement is not fulfilled because $\Pi$ (which is negative) is too large, we regulate $\Pi$ such that $f(0)=0$. In this special situation one can further compute

$$
\frac{d f}{d e}(e=0)=\left(c_{s}^{2}-1\right) M^{0}-\Pi .
$$

If $\left.\frac{d f}{d e}\right|_{e=0} \leq 0, e=0$ is the solution. For $\left.\frac{d f}{d e}\right|_{e=0}>0$, there will be a positive energy density solution. Without $\Pi,\left.\frac{d f}{d e}\right|_{e=0}$ is always less than 0 because the square of the speed of sound is always smaller than 1 .

Once these two boundary conditions are set up, it is guaranteed that there will be at least one solution of Eq. (33) with positive energy density. Newton's root finding method is a very efficient in finding this solution with a minimal number of iterations. To ensure numerical stability and optimal efficiency, we use the fact that to fairly good approximation the pressure is roughly proportional to the energy density. We rewrite Eq. (34) as,

$$
f(e)=\left(M^{0}-e\right)\left(M^{0}+\frac{P}{e} e+\Pi\right)-\left(\left(M^{1}\right)^{2}+\left(M^{2}\right)^{2}\right)
$$

and use that $\tilde{c}_{s}^{2}=\frac{P}{e}$ has a very weak dependence on $e$. This turns the condition $f(e)=0$ into approximately a quadratic equation with solution

$$
e=\frac{-\left(M^{0}\left(1-\tilde{c}_{s}^{2}\right)+\Pi\right) \pm \sqrt{\left(M^{0}\left(1-\tilde{c}_{s}^{2}\right)+\Pi\right)^{2}+4 \tilde{c}_{s}^{2}\left(M^{0}\left(M^{0}+\Pi\right)-M\right)}}{2 \tilde{c}_{s}^{2}} .
$$

To identify the correct sign, we note that for $M=0$ we must recover $e=M^{0}$. Therefore,

$$
e=\frac{-\left(M^{0}\left(1-\tilde{c}_{s}^{2}\right)+\Pi\right)+\sqrt{\left(M^{0}\left(1-\tilde{c}_{s}^{2}\right)+\Pi\right)^{2}+4 \tilde{c}_{s}^{2}\left(M^{0}\left(M^{0}+\Pi\right)-M\right)}}{2 \tilde{c}_{s}^{2}} .
$$

This equation is the most efficient satisfying form for applying Newton's method, and it is implemented in VISHNew.

Once Eq. 27] has been solved for $e$, the flow velocity can be calculated from

$$
u^{0}=\left(\frac{M^{0}+P+\Pi}{e+P+\Pi}\right)^{1 / 2}
$$

where $P=P(e)$ is obtained from the EOS. Please note that calculating $u^{0}$ instead of $v$ is numerically more stable when $v \rightarrow 1$. Since $u^{0} \geqslant 1$, this requires $M^{0} \geqslant e$. So $M^{0}$ should be set as an upper limit for $e$ when intreating $e$ using Newton's root finding routine. Similarly,

$$
u^{i}=\frac{M^{i}}{\sqrt{M^{0}+P+\Pi} \sqrt{e+P+\Pi}}(i=1,2) .
$$

One can check that if $e$ is the exact solution of Eq. 33, the flow velocity components Eq. 396 and Eq. (40) satisfy the normalization constraint

$$
\left(u^{0}\right)^{2}-\left(u^{1}\right)^{2}-\left(u^{2}\right)^{2}=1 .
$$




\subsubsection{With baryon density current}

The derivations above assumed zero net baryon density where the pressure is only a function of the local energy density. In order to deal with the more general cases of non-zero conserved charge current in the future, we now consider the situation where the baryon current is not zero.

In this case the pressure is a function of both the local energy density and the local net baryon density: $P=P(e, n)$. For the baryon current, we have the additional hydrodynamic equation

$$
\partial_{\mu} j^{\mu}=0
$$

where ( $V^{\mu}$ is the heat flow vector)

$$
j^{\mu}=n u^{\mu}+V^{\mu} .
$$

Now, the problem of implementing the EOS presents itself as follows: knowing $j^{0}, T^{00}, T^{01}$ $T^{02}, T^{03}$ and the EOS, we would like to solve for 5 unknowns $n, e, u^{\mu}$. We have the following 5 equations:

$$
\begin{gathered}
M^{0}=(e+P+\Pi)\left(u^{0}\right)^{2}-P-\Pi, \\
M^{i}=(e+P+\Pi) u^{0} u^{i},(i=1,2,3), \\
j^{0}=n u^{0}+V^{0} .
\end{gathered}
$$

We can no longer solve for $e$ easily, because the pressure now depends on both $e$ and $n$. The equations for $e$ and $n$ are coupled with each other. To decouple these two equations, we need to know the actual functional dependence for $P(e, n)$. In such a situation, it is easilier to solve for $v$ or $u^{0}$ first. For $v$, we have solve the following equation:

$$
v=\frac{M}{M^{0}+P+\Pi}
$$

where $M=\sqrt{\left(M^{1}\right)^{2}+\left(M^{2}\right)^{2}+\left(M^{3}\right)^{2}}$. For the pressure from the EOS, we need to work out

$$
\begin{gathered}
e=M^{0}-v M \\
n=\left(j^{0}-V^{0}\right) \sqrt{1-v^{2}}
\end{gathered}
$$

To solve Eq. 477 we define

$$
f(v)=v\left(M^{0}+P+\Pi\right)-M .
$$

We have the boundary conditions

$$
f(0)=-M \leqslant 0
$$

and

$$
f(1)=M^{0}+P+\Pi-M .
$$

Imposing $f(1) \geqslant 0$ will ensure an odd number of solutions. From Eqs. (48) and Eq. (49) we see that $e$ and $n$ are roughly linear in $v$, which means that $P$ is also roughly linear in $v$. So we expect to have only one solution. Please note that since $v$ is bounded between 0 and 1 , we need to ensure high precision of the solution, otherwise $u^{\mu}$ will not be accurate, especially when $v \rightarrow 1$.

Once $v$ is solved and thus $e$ and $n$ are known from Eqs. (48) and (49, $, v_{x}, v_{y}, v_{z}$ can be solved easily from $M^{1}, M^{2}, M^{3}$,

$$
v^{i}=\frac{M^{i}}{M^{0}+P(e, n)+\Pi},(i=1,2,3) .
$$


In order to use Newton's method to find the root of the key equation 47, we can reorganize it as follows:

$$
f(v)=v\left(M^{0}+\tilde{c}_{s}^{2}\left(M^{0}-v M\right)+\Pi\right)-M .
$$

Eq. 54 can be considered as an approximatly quadratic equation for $v$. The condition $f(v)=0$ it has the solutions

$$
v=\frac{\left(M^{0}\left(1+\tilde{c}_{s}^{2}\right)+\Pi\right) \pm \sqrt{\left(M^{0}\left(1+\tilde{c}_{s}^{2}\right)+\Pi\right)^{2}-4 \tilde{c}_{s}^{2} M^{2}}}{2 \tilde{c}_{s}^{2} M} .
$$

The correct sign is found by checking the limit $M \rightarrow 0$, when $v$ approaches to zero. This selects the negative sign in Eq. 55, which can thus be rewritten as

$$
v=\frac{2 M}{\left(M^{0}\left(1+\tilde{c}_{s}^{2}\right)+\Pi\right)+\sqrt{\left(M^{0}\left(1+\tilde{c}_{s}^{2}\right)+\Pi\right)^{2}-4 \tilde{c}_{s}^{2} M^{2}}} .
$$

The advantage of Eq. (56) is that the right hand side is only very weakly dependent on $v$ as long as $\tilde{c}_{s}^{2}$ is approximately a constant which is true over a very wide range of energy densities for s95p EOS. Additionally, it is numerically stable in the limit $M \rightarrow 0$. Similarly, we can find a solution for $u^{0}$ :

$$
u^{0}=\frac{1}{\sqrt{1-v^{2}}}
$$

Eqs. 56 and 57 in principle give consistent solutions for $v$ and $u^{0}$. In practice, inevitable numerical errors render the use of Eq. (56) preferable for small velocities $v \rightarrow 0$, while Eq. (57) should be used for $v \rightarrow 1$. Let us see why this is the case:

If we solve $u^{0}$ from Eq. 57) and write the numerical solution as $\tilde{u}^{0}=u^{0}+\Delta u$ where $u^{0}$ is the exact solution and $\Delta u$ is the numerical error, the numerical error for $v$ can be estimated as,

$$
\Delta v=\frac{d v}{d u^{0}} \Delta u^{0}=\frac{\Delta u^{0}}{\left(\tilde{u}^{0}\right)^{2} \sqrt{\left(\tilde{u}^{0}\right)^{2}-1}}\left(1+O\left(\Delta u^{0}\right)\right) .
$$

In this situation, $\Delta v$ becomes small, $\Delta v \ll \Delta u^{0}$, for large flow velocity, $\tilde{u}^{0} \rightarrow+\infty$. On the other hand, when $v \rightarrow 0$ and $\tilde{u}^{0} \rightarrow 1$, the numerical error for $v$ is amplified by a factor $\frac{1}{\sqrt{\left(\tilde{u}^{0}\right)^{2}-1}}$ compared to $\Delta u^{0}$, which is not good. Therefore Eq. 57) is numerically stable for $v \rightarrow 1$ and unstable for $v \rightarrow 0$.

The opposite is true for Eq. [56, Writing the numerical solution of Eq. (56) as $\tilde{v}=v+\Delta v$, we find,

$$
\Delta u=\frac{d u^{0}}{d v} \Delta v=\frac{\tilde{v}}{\left(\sqrt{1-\tilde{v}^{2}}\right)^{3}} \Delta v .
$$

In this case, $\Delta u^{0} \ll \Delta v$ for small $\tilde{v} \rightarrow 0, \Delta u^{0} \ll \Delta v$, which is favorable. On the other hand, for large velocity, $\tilde{v} \rightarrow 1$, the error in $u^{0}, \Delta u^{0} \sim \frac{1}{\left(\sqrt{1-\tilde{v}^{2}}\right)^{3}} \Delta v$, is amplified by a factor $\frac{1}{\left(\sqrt{1-\tilde{v}^{2}}\right)^{3}}$ relative to $\Delta v$, making $u^{0}$ numerically unstable.

In the actual numerical implementation, we solve both Eq. (56) and Eq. (57), but we then select the preferred solution according to the magnitude of the resulting velocity. The transition point from one choice to the other happens at

$$
\frac{\tilde{v}}{\left(\sqrt{1-\tilde{v}^{2}}\right)^{3}}=\frac{1}{\left(\tilde{u}^{0}\right)^{2} \sqrt{\left(\tilde{u}^{0}\right)^{2}-1}}
$$


with the relation $\tilde{u}=\frac{1}{\sqrt{1-\tilde{r}^{2}}}$. The numerical solution of Eq. 600 is $\tilde{v}=0.563624$ or $\tilde{u}^{0}=1.21061$. For velocities smaller than this critical value, we use the solution for $v$ from Eq. [56, while for larger velocities, we should select the solution for $u^{0}$ from Eq. 577) as the more reliable one.

\subsection{Numerical check for VISHNew using semi-analytic solutions}

In [58, 59], the authors derived $S O(3) \otimes S U(1,1) \otimes Z_{2}$ invariant ("Gubser symmetric") solutions of ideal relativistic conformal fluid dynamics which couple boost-invariant longitudinal expansion to an azimuthally symmetric transverse expansion. We first use this $(1+1)$-d solution to check the ideal hydrodynamic mode in VISHNew. We start our ideal hydrodynamic simulation with Gubser's solution for the energy density and flow velocity at $\tau=1.0 \mathrm{fm} / \mathrm{c}$ and compare results at later proper time with Gubser's analytic solution. Fig. 9 shows excellent agreement between our simulations and the analytical solution.
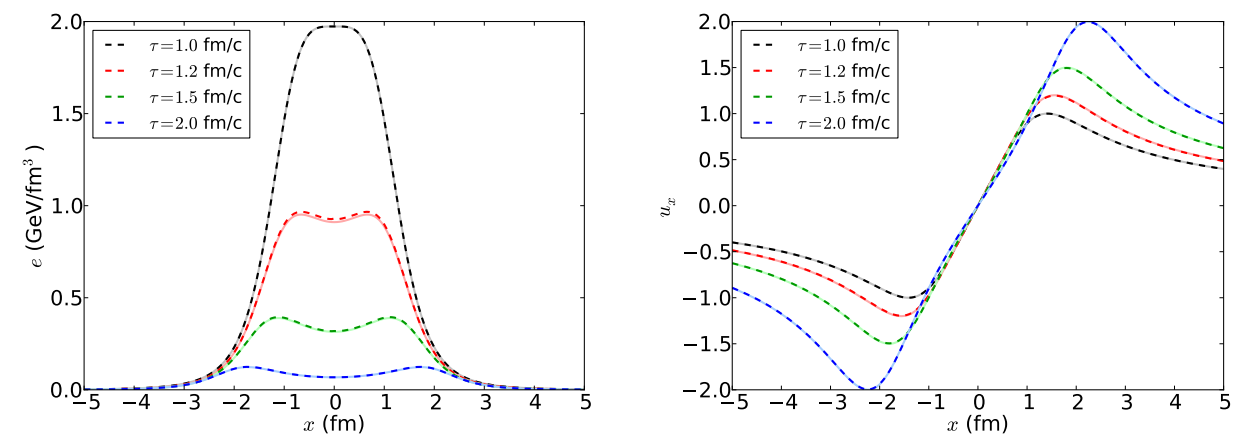

Figure 9: Comparison of the numerical solution (dark dashed lines) for (1+1)-d ideal fluid dynamical evolution with Gubser symmetry with Gubser's analytical results (light solid lines).

For viscous hydrodynamics, Marrochio et al. [60] have used the same symmetry argument developed by Gubser and constructed a nontrivial semi-analytic solution of the Israel-Stewart equations for (1+1)-d expansion with Gubser symmetry. In order to use this solution as a check of the VISHNew code, we have to change the source term in the transport equation of shear stress tensor as specified in [60],

$$
\Delta^{\mu \alpha} \Delta^{\nu \beta} D \pi_{\alpha \beta}=-\frac{1}{\tau_{\pi}}\left(\pi^{\mu \nu}-2 \eta \sigma^{\mu v}\right)-\frac{4}{3} \pi^{\mu v} \theta
$$

The difference between Eqs. 287 and (61) only appears in third and higher orders in velocity gradients. However, since these gradients are large for the Gubser profile, the difference is noticeable and would be visible even if VISHNew were a perfect numerical algorithm. We use the same parameters as in [60] (described below) to test our viscous hydrodynamic simulations. We start the simulation at $\tau=1.0 \mathrm{fm} / \mathrm{c}$ and use the semi-analytical solutions from [60] at $\tau=1.0$ $\mathrm{fm} / \mathrm{c}$ as the initial conditions for our simulations. We use an ideal massless gas equation of state $e=3 P$, with

$$
e=N_{c} \times\left(16+\frac{7}{2} \times 3 N_{f}\right) \times \frac{\pi^{2}}{90} T^{4}
$$



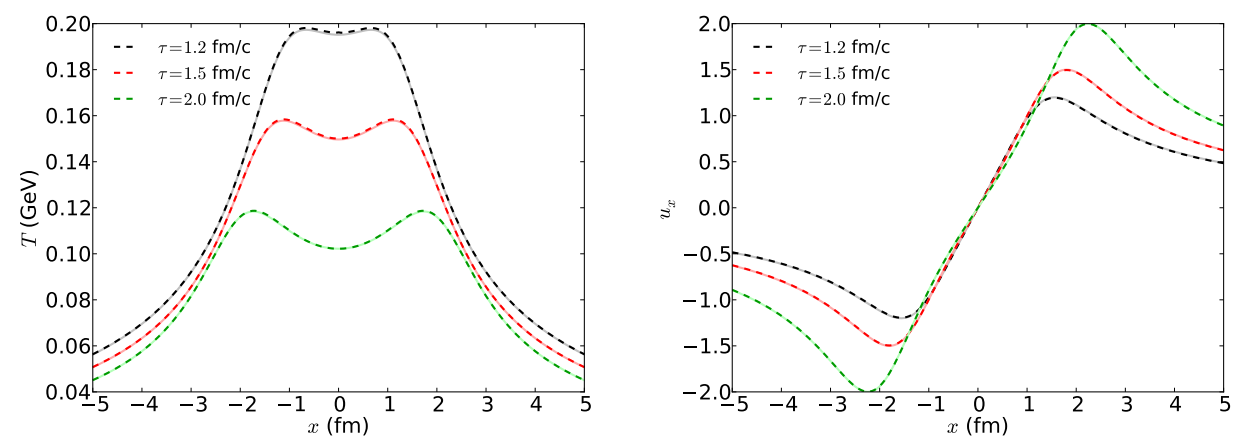

Figure 10: Comparison of the temperature and flow velocity evolution from VISHNew (dark dashed) with the semianalytical solutions from [60] (light solid).
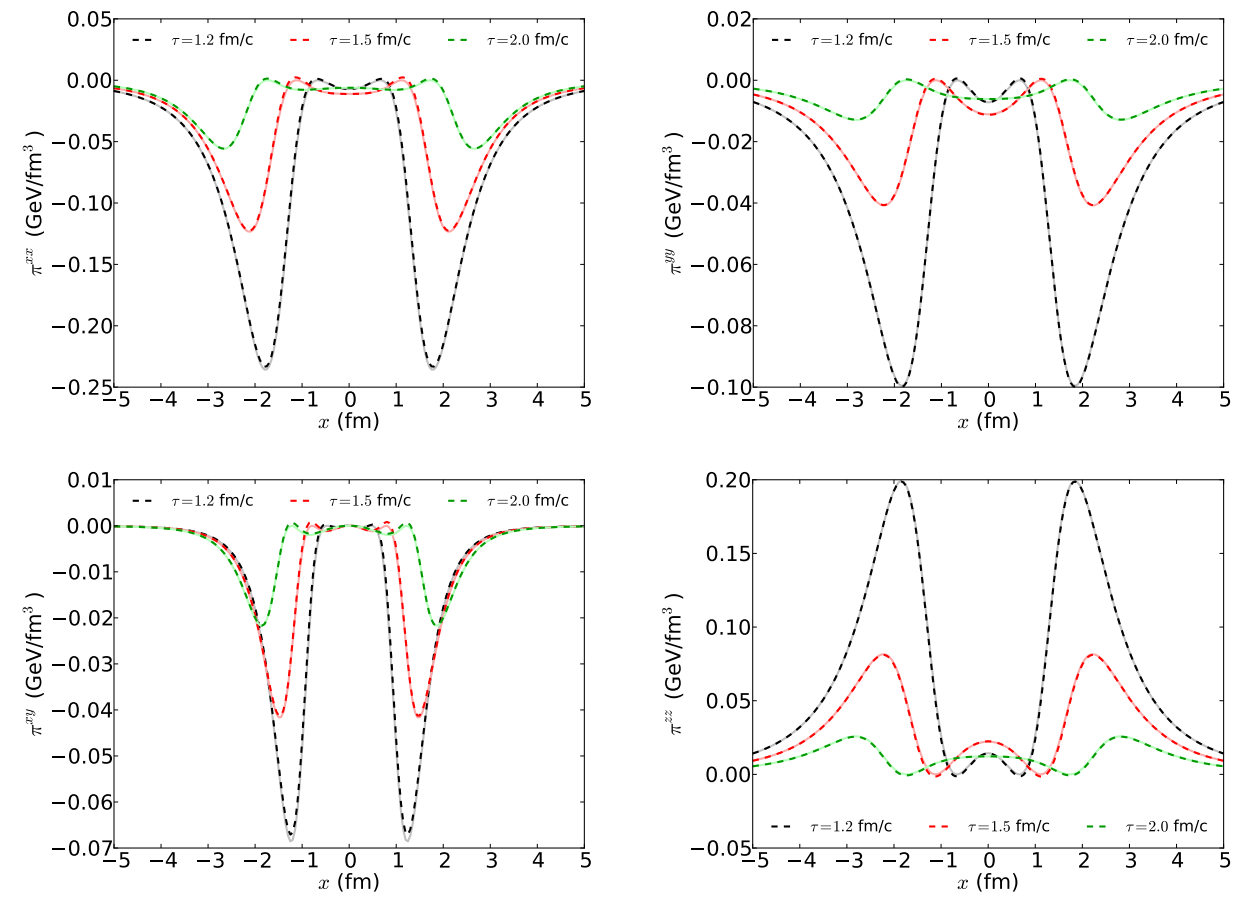

Figure 11: The evolution of individual components of the shear stress tensor from VISHNew (dark dashed) compared with the semi-analytical solutions from [60] (light solid).

using $N_{c}=3$ for the number of colors and $N_{f}=2.5$ for the number of flavors. We set the specific shear viscosity to $\eta / s=0.2$ and its corresponding relaxation time to $\tau_{\pi}=5 \eta /(T s)$.

In Figs. 10 and 11, we compare our numerical calculations with the semi-analytical solutions from [60] for the evolution of the local temperature, flow velocity, and shear stress tensor. For all hydrodynamic quantities we find very good agreement of our VISHNew simulations with the semi-analytical results. 


\subsection{Stabilizing VISHNew against numerical fluctuations in the viscous shear}

VISHNew solves the minimum set of second order viscous hydrodynamic equations. The shear stress tensor is evolved according to Eq. 28, which only includes spatial gradients up to second order. Such a truncation of the gradient expansion converges and gives good approximations only when higher order gradient terms are negligible. When we perform event-by-event hydrodynamic simulations, the fluctuating initial conditions usually feature large spatial gradients in the transverse plane. Under such conditions, the missing higher order gradient corrections to Eq. 28) have the potential to grow large during the hydrodynamic evolution, and not including them in the code may eventually drive the whole numerical simulation into instability. However including all the higher order gradient terms in the transport equation for $\pi^{\mu v}$ is not practical. It would require the knowledge of the corresponding higher order transport coefficients, which are poorly constrained both theoretically and experimentally.

Therefore, staying within the framework of second order viscous hydrodynamics, we apply a regulation to the shear stress tensor that aims to suppress numerical instabilities caused by large spatial gradients. Similar regulation procedures are also performed in Refs. [9, 30]. In general, for second order viscous hydrodynamics to be valid, $\pi^{\mu \nu}$ must to satisfy the following criteria:

1. $\pi^{\mu v}$ should be smaller than the ideal part of the energy momentum tensor, $T_{0}^{\mu v}=e u^{\mu} u^{v}$ $P \Delta^{\mu v}$. To implement this we compare the following Lorentz invariant quantities,

$$
\operatorname{Tr}\left(\pi^{2}\right)=\pi^{\mu v} \pi_{\mu \nu} \text { and } T_{0}^{\mu v} T_{0 \mu \nu}=e^{2}+3 P^{2} .
$$

Consistency for our theoretical framework requires

$$
\pi^{\mu v} \pi_{\mu \nu} \ll e^{2}+3 P^{2}
$$

2. $\pi^{\mu \nu}$ should be traceless:

$$
\pi_{\mu}^{\mu}=0
$$

3. $\pi^{\mu v}$ should be perpendicular to $u^{\mu}$ :

$$
\pi^{\mu v} u_{v}=0
$$

VISHNew evolves all seven non-vanishing components of $\pi^{\mu \nu}, \pi^{\alpha \beta}$ (where $\alpha, \beta=\tau, x, y$ ) and $\pi^{\eta \eta}$, independently without enforcing the conditions 2 and 3. Checking the validity of Eqs. (64) and 65 for the numerically evolved $\pi^{\mu v}$ thus amounts to a check of the numerical accuracy of our code. In actual calculations, there are limits to the numerical accuracy of $\pi^{\mu v}$ so we choose a small number $\xi_{0} \ll 1$ as the "relative numerical zero" and replace conditions 2 and 3 by

$$
\pi_{\mu}^{\mu} \leq \xi_{0} \sqrt{\pi^{\mu v} \pi_{\mu v}} \text { and } \pi^{\mu v} u_{v} \leq \xi_{0} \sqrt{\pi^{\mu v} \pi_{\mu \nu}}, \forall \mu
$$

The vector $\pi^{\mu v} u_{v}$ should be component-wise zero (in any frame), therefore all its components should be compared to the "relative numerical zero" multiplied by $\sqrt{\pi^{\mu \nu} \pi_{\mu \nu}}$ (for dimensional reasons). Here we use the scalar $\sqrt{\pi^{\mu \nu} \pi_{\mu \nu}}$ as a measure for the magnitude of the $\pi^{\mu v}$ tensor that sets the scale (via the factor $\xi_{0}$ ) for how close the numerical result is to zero. that ${ }^{6}$

In practice, to ensure that Eq. 63 is satisfied, we choose a number $\rho_{\max } \ll 1$ and require

$$
\sqrt{\pi^{\mu v} \pi_{\mu \nu}} \leq \rho_{\max } \sqrt{e^{2}+3 p^{2}}
$$

\footnotetext{
${ }^{6} \rho_{\max } \ll 1$ corresponds to the required "«" condition in Eq. 66 ; $\rho_{\max }=\infty$ corresponds to no constraint at all.
} 
In our simulations, we found, that this condition is sometimes violated during the early stage and/or in the dilute regions outside the freeze-out surface (see at Fig. 14 for an example). The violation of Eq. (63) in these regions do not have much influence on the dynamical behavior of the QGP in the physical region inside the freeze-out surface; however, if left untreated, such violations lead to accumulating numerical errors that eventually cause the evolution code to break down at later times. For these reasons, in the following we develop a systematic treatment that suppresses large viscous terms. This stabilizes the code with negligible effects on the physics and negligible extra numerical cost.

We enforce a continuous systematic regulation on $\pi^{\mu v}$ in each time step on the whole lattice by replacing $\pi^{\mu \nu}$ by $\hat{\pi}^{\mu \nu}$ :

$$
\pi^{\mu \nu} \rightarrow \hat{\pi}^{\mu \nu} \equiv \pi^{\mu \nu} \frac{\tanh (\rho)}{\rho},
$$

where $\rho$ is the largest quantity at each lattice point among the following:

$$
\frac{\sqrt{\pi^{\mu v} \pi_{\mu \nu}}}{\rho_{\max } \sqrt{e^{2}+3 p^{2}}}, \frac{\pi_{\mu}^{\mu}}{\xi_{0} \rho_{\max } \sqrt{\pi^{\mu v} \pi_{\mu \nu}}} \text {, or } \frac{\pi^{\mu v} u_{v}}{\xi_{0} \rho_{\max } \sqrt{\pi^{\mu v} \pi_{\mu \nu}}}, \forall \mu
$$

It is easy to check that $\hat{\pi}^{\mu v}$ satisfies Eq. (67), and that it is close to $\pi^{\mu v}$ where no modifications are needed; that is, when the left hand side of the inequality in Eq. 67) is small compared to the right hand side, Only at those grid points where $\pi^{\mu \nu}$ violates or is close to violating the inequality 67) will it be strongly modified; if this is the case, all components of $\pi^{\mu v}$ are suppressed by the same factor.

Because smoother flow velocity profiles give smaller $\pi^{\mu \nu}$, the systematic suppression of $\pi^{\mu \nu}$ can be understood as locally replacing sharp jumps in the flow profile by smoother pieces; the regulation process is therefore an implicit and automatic way of smoothing profiles. This treatment allows us to perform hydrodynamic calculations using very bumpy initial conditions, including those using disk-like nucleons that have density discontinuities. Without this regularization VISHNew breaks down for such initial conditions. We note that typically no regulations are required inside the freeze-out surface at later times; shear viscosity leads to dynamical smoothing of initial fluctuation by dissipation, suppressing sharp velocity gradients and large values of $\pi^{\mu \nu}$ as time proceeds. Regulation remains necessary in the dilute region outside the freeze out surface where $e$ and $P$ (which for massless degree of freedom both fall like $T^{4}$ ) fall faster than $\pi^{\mu \nu}$ (which falls only like $T^{3}$ ).

In our calculations, we take $\xi_{0}=0.1$. If we choose smaller $\xi_{0}$ in the simulations, we overkill the physical viscous damping effects. In the following section, we show tests invoking several choices of $\xi_{0}$ and their influence on the final observables. During our tests we found that $\rho_{\max }$ is best chosen to be a value between $1-10$. By choosing $\rho_{\max }$ of order unity or larger, we reduce the regulation strength in each step to the point where the code is numerically stable with minimum modification. (Note that this implies that the code may run in a domain where the strong inequality Eq. 63 is not satisfied, i.e. second order viscous hydrodynamics may not be strictly valid (see [61] for a related study).)

\subsection{Hydrodynamic evolution with regulation}

In this section, we study the sensitivity of final hadronic observables on the choice of the $\xi_{0}$ parameter used in the $\pi$ regulation routine. For these tests, we choose MC-Glauber initial conditions for $\mathrm{Pb}+\mathrm{Pb}$ collisions at $\sqrt{s}=2760 A \mathrm{GeV}$ at $20-30 \%$ centrality, using $\eta / s=0.20$. We simulate 200 events for every choice of $\xi_{0}$. 


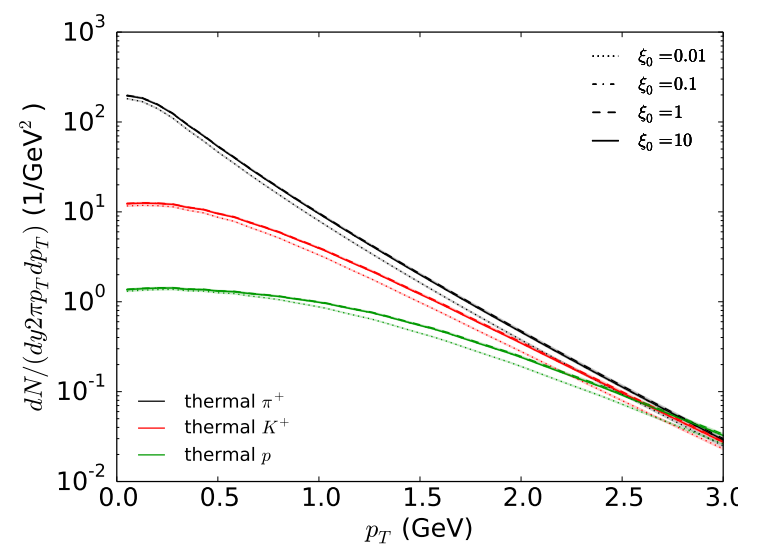

Figure 12: Thermal particles' $p_{T}$ spectra for different choice of $\xi_{0}$ used in the regulation trigger routine.

In Fig. 12, we show the $p_{T}$-spectra for thermal $\pi^{+}, K^{+}$, and protons, with different values of $\xi_{0}$ used in the trigger routine for the $\pi^{\mu v}$ regulation. We find that for $0.1 \leq \xi_{0} \leq 10$, there is no noticeable difference between different choices of $\xi_{0}$ used in the simulations. Only for the very small value $\xi=0.01$ we see an effect: particle spectra get steeper, and the yield decreases. This means that the system generates less entropy and radial flow during the evolution, which indicates that the shear viscous effects in the simulations are suppressed too strongly by the regulations.

In Fig. 13, we show results for the $p_{T}$-differential anisotropic flows $v_{2}$ to $v_{5}$. They show a larger sensitivity to the choice of $\xi_{0}$ than the single particle spectra. For $0.1 \leq \xi_{0} \leq 10$, the $v_{n}$ of thermal particles agree reasonably well with each other within the statistical error bands. But for $\xi_{0}=0.01$, the regulation again over-suppresses the viscous effects, which damp the anisotropic flow of the system.

From this parameter study we conclude that the final hadronic observables are not sensitive to the choice of $\xi_{0}$ as long as we keep it in the range $0.1 \leq \xi_{0} \leq 10$. For $\xi_{0}$ larger than 10, the code becomes numerically unstable due to too strong violations of the criteria that ensure validity of the second order viscous hydrodynamic description. For $\xi_{0}$ smaller than 0.1 , the regulation routine seems to over kill the shear viscous effects in the system, thereby altering the physics by using an effective shear viscosity that is much smaller than the input value. In our application of VISHNew, we therefore always use $\xi_{0}$ in the range $0.1 \leq \xi_{0} \leq 10$.

In order to quantify the quality of reliability of our second order viscous hydrodynamic approach we can monitor the inverse Reynolds number and Knudsen number associated with shear stress, defined as [61]:

$$
R_{\pi}^{-1}=\frac{\sqrt{\pi^{\mu v} \pi_{\mu v}}}{\mathcal{P}}
$$

and

$$
K n_{\theta}=\frac{\lambda_{\mathrm{mfp}}}{L_{\mathrm{hydro}}}=\tau_{\pi} \theta=5 \frac{\eta \theta}{s T} .
$$

Here $\mathcal{P}$ is the thermal pressure, $\tau_{\pi}$ is the shear relaxation time, and $\theta$ is system expansion rate. As long as $R_{\pi}^{-1}$ and $K n_{\theta}$ are smaller than 1, the system behaves like a fluid with low viscosity. For $R_{\pi}^{-1} \gg 1$, the behavior is more dissipative and viscous hydrodynamics is no long a good description. And for $K n_{\theta}>1$, the collision rate is not high enough to keep the system to stay near 

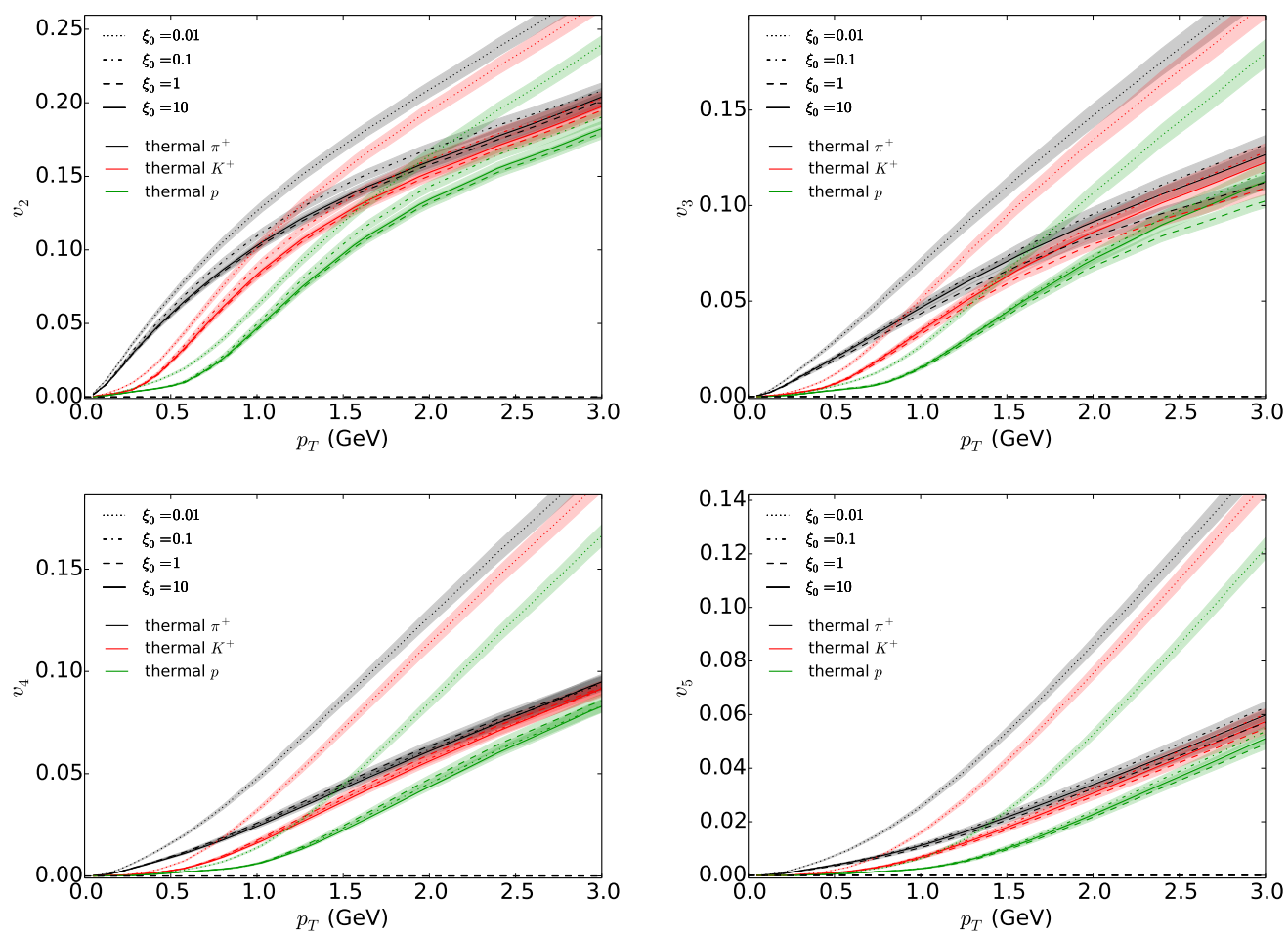

Figure 13: Thermal particles' $p_{T}$-differential $v_{n}$ for different choice of $\xi_{0}$ used in the regulation trigger routine. Statistical errors are indicate as shaded bands in the plots.

thermal equilibrium. In Fig. 14, we show a contour plot of the evolution of the inverse Reynolds number and Knudsen number in our hydrodynamic simulation at $y=0$ in the transverse plane. In the left panels we start with a smooth event-averaged initial condition; in the right panels we show results for a fluctuating initial profile. The white points indicate the position of the kinetic freezeout surface. We notice that the largest inverse Reynolds numbers are encountered at early times of the hydrodynamic evolution or outside the freeze-out surface for both smooth and fluctuating initial conditions. And we find similar situation for the Knudsen number evolution. As time goes on, the magnitude of the shear stress tensor decreases, and the relativistic hydrodynamic modeling becomes more and more reliable. At very early times the use of viscous hydrodynamics becomes questionable, especially for fluctuating initial profiles. Please note that with a constant $\eta / s$, our estimation of the Knudsen number $K n_{\theta}=5 \frac{\eta}{s T} \theta$ might not be reliable in dilute hadronic regions. In principle the value of $\eta / s$ will increases as temperature decreases. So the Knudsen number should increase in the low density region in the hadron gas phase.

\section{Cooper-Frye freezeout using is and particle sampler iSS}

The name "iS" stands for "iSpectra"; iS is a fast Cooper-Frye particle momentum distribution calculator along the conversion surface. Its output is a continuous function, evaluated 

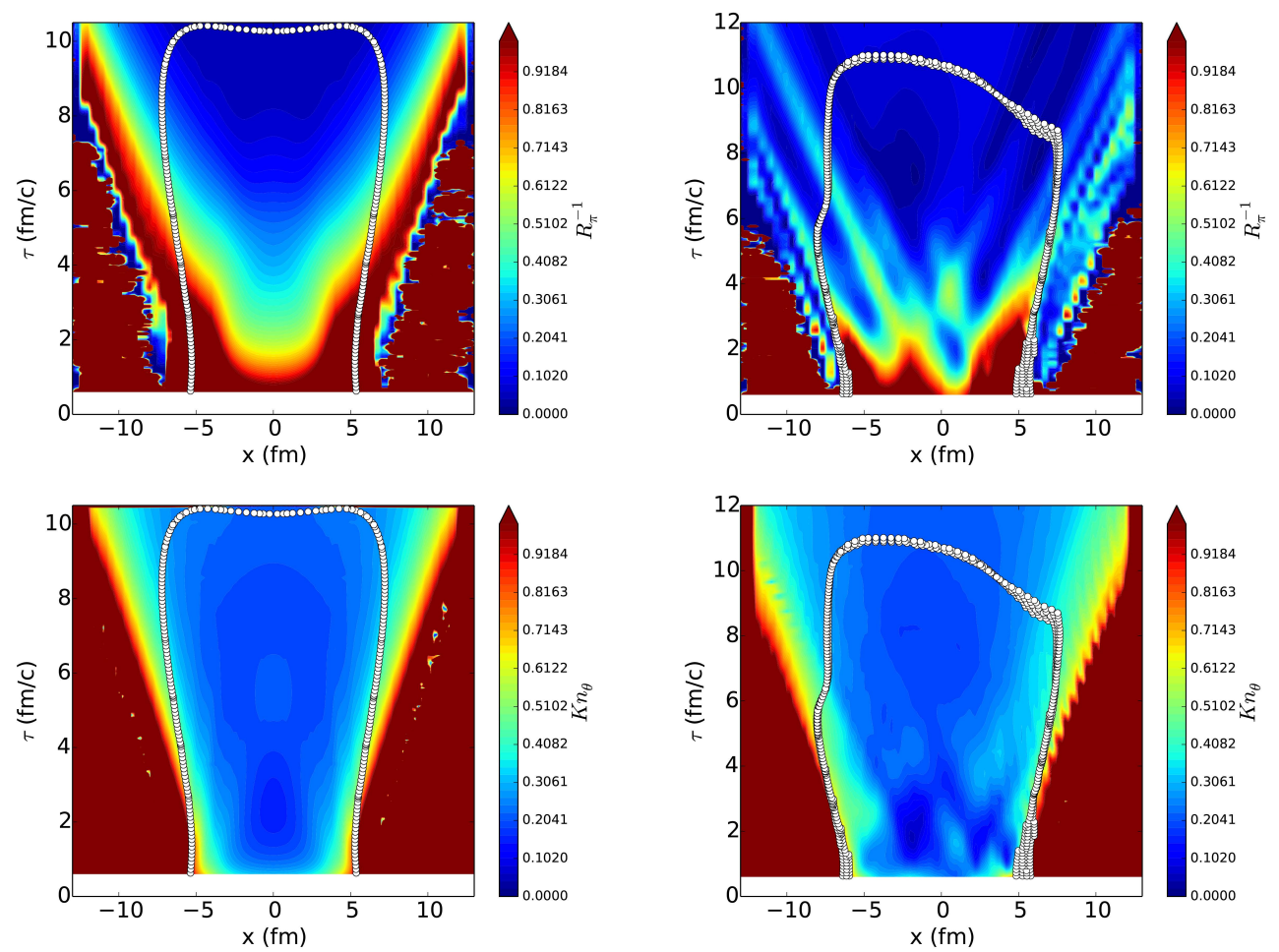

Figure 14: Contour plot for the evolution of the inverse Reynolds number (upper panels) and Knudsen number (lower panels) in viscous hydrodynamic simulations with $\eta / s=0.20$ at $20-30 \%$ LHC energy. The white points indicate the kinetic freeze surface at $T_{\mathrm{dec}}=120 \mathrm{MeV}$.

at discrete momenta provided by the user, for the invariant momentum distributions of the desired hadron species. The code "iSS", whose name stands for "iSpectraSampler", goes one step further to generate individual particles samples, using the calculated particle momentum distributions as the relative emission probability. iSS is an "event generator" which generates a complete collision event of emitted hadrons, similar to the events created in the experiment. Both codes are written keeping the following factors in mind:

- Readability and extendability. The most important goal is to create a cleanly written framework that calculates particle momentum distributions and performs sampling, whose components and output can be used easily for further physics analyses and tests of new physical ideas. To achieve this, the entire program is divided into modules according to their functionalities, the structures and the algorithms are documented with comments, and long but informative names are chosen for variables and function names.

- Efficiency. Both the iS and iSS codes are written aiming for intensive event-by-event calculations where every CPU cycle counts. To achieve the necessary degree of efficiency, much effort is put into optimizing the algorithms at different levels of the calculations.

- Easy maintainability and re-usability. The framework is divided into different carefully chosen functionality modules, for better interoperability and to maximize re-usability. 


\subsection{Cooper-Frye freeze-out}

The particle emission function that implements sudden decoupling from a surface element $d^{3} \sigma$ located on a freeze-out hyper-surface $\Sigma\left(x^{\mu}\right)$ is given by the Cooper-Frye formula,

$$
E \frac{d N}{d^{3} p}\left(x^{\mu}, p^{\mu}\right)=\frac{g}{(2 \pi)^{3}} p^{\mu} d^{3} \sigma_{\mu}\left(f_{0}\left(x^{\mu}, p\right)+\delta f\left(x^{\mu}, p^{\mu}\right)\right),
$$

where $g$ is the spin degeneracy, $d^{3} \sigma_{\mu}=\left(\cosh \eta_{s},-\partial \tau / \partial x,-\partial \tau / \partial y,-\sinh \eta_{s}\right) \tau d x d y d \eta_{s}$ is the infinitesimal surface element on $\Sigma\left(x^{\mu}\right)$ for systems with longitudinal boost-invariance, and $f_{0}\left(x^{\mu}, p\right)$ is local thermal equilibrium distribution function. $\delta f\left(x^{\mu}, p^{\mu}\right)$ represents the deviation from local thermal equilibrium due to viscous effect and takes the following form,

$$
\delta f\left(x^{\mu}, p^{\mu}\right)=f_{0}\left(x^{\mu}, p\right)\left(1 \pm f_{0}\left(x^{\mu}, p\right)\right) \frac{\pi_{\mu \nu} \hat{p}^{\mu} \hat{p}^{v}}{2(e+P)} \chi\left(\frac{p \cdot u}{T}\right)
$$

where $\hat{p}^{\mu}=p^{\mu} /(p \cdot u)$ and $\chi(p \cdot u / T)=(p \cdot u / T)^{\alpha}$ with $1 \leq \alpha \leq 2$. Integrating the emission function over the freeze-out surface we obtain particle momentum distribution

$$
\frac{d N}{d y p_{T} d p_{T} d \phi_{p}}=\int_{\Sigma} \frac{g}{(2 \pi)^{3}} p^{\mu} d^{3} \sigma_{\mu}\left(f_{0}\left(x^{\mu}, p\right)+\delta f\left(x^{\mu}, p^{\mu}\right)\right)
$$

The azimuthally averaged $p_{T}$-spectrum is given by,

$$
\frac{d N}{2 \pi d y p_{T} d p_{T}}=\int \frac{d \phi_{p}}{2 \pi} \frac{d N}{d y p_{T} d p_{T} d \phi}
$$

while the anisotropic flow coefficients are computed from,

$$
\begin{gathered}
V_{n} \equiv v_{n} e^{i n \Psi_{n}}=\frac{\int p_{T} d p_{T} d \phi_{p} e^{i n \phi_{p}} d N /\left(d y p_{T} d p_{T} d \phi_{p}\right)}{\int p_{T} d p_{T} d \phi_{p} d N /\left(d y p_{T} d p_{T} d \phi_{p}\right)}, \\
V_{n}\left(p_{T}\right) \equiv v_{n}\left(p_{T}\right) e^{i n \Psi_{n}}=\frac{\int d \phi_{p} e^{i n \phi_{p}} d N /\left(d y p_{T} d p_{T} d \phi_{p}\right)}{\int d \phi_{p} d N /\left(d y p_{T} d p_{T} d \phi_{p}\right)} .
\end{gathered}
$$

To optimize the efficiency of the numerical calculations, gaussian quadrature points are used for the variables $p_{T}, \phi_{p}$, and $\eta_{s}$. Further optimization for performing the numerical integral in Eq. (73) involves adjusting the order of the integration loops, using local variables, pre-tabulating mathematical functions, etc. The resulting code is is $\sim 7$ times faster compared to its ancestor AZSpectra [62].

\subsection{Methodology for particle sampling}

The particle emission function from the Cooper-Frye formula Eq. (71) can be regarded as the probability of emitting particle from a given freeze-out fluid cell with specified momentum. The program iSS uses this probability to generate sets of momenta and positions for actual particles emitted at the end of the hydrodynamic simulation. This information is then used as input for the following microscopic hadron cascade simulation. In the sampling procedure, we employ two well-known statistical sampling methods, the inverse cumulative distribution function (CDF) method and the direct probability distribution function (PDF) method, the latter is also known as the acceptance and rejection method. 


\subsubsection{Purely numerical approach}

The straightforward (although not necessarily the fastest) approach is to compute all the required quantities numerically.

For a given particle species, the average total number of particles per unit rapidity, $d N / d y$, is calculated by numerically integrating Eq. (71) over all freeze-out fluid cells and all particle transverse momenta $\vec{p}_{T}$. During the numerical integration, an inverse CDF can be built up with negligible numerical cost for latter efficient sampling. However, in practice, the inverse CDF for a full set of spatial and momentum variables is memory demanding. In order to sample such a multi-dimensional probability distribution function, we divide the random variables into two groups and use efficient specific sampling methods to handle each of them. It is natural to group the spatial information $\left(\tau, \vec{x}_{\perp}, \eta_{s}\right)$ for the sampled particles into one set of random variables, and their momenta $\left(p_{\perp}, \phi_{p}, y\right)$ into the other. Dividing the random variables into two groups allows us to perform the sampling in different order and with different methods.

One way to proceed is to first sample the spatial information, $\left(\tau, \vec{x}_{\perp}, \eta_{s}\right)$, using the inverse CDF method. Along with calculating the particle yield $d N / d y$, (see above) we build up the inverse CDF for the particle's spatial variables, $\left(\tau, \vec{x}_{\perp}, \eta_{s}\right)$, by integrating Eq. 71 over the transverse momentum, $\left(p_{\perp}, \phi_{p}\right)$. For a collision event at top RHIC energy, the typical size of the array to store the inverse $\mathrm{CDF}$ is about 30,000 freeze-out fluid cells in the transverse plane times 40 points along the $\eta_{s}$ direction. Once we have the particles' spatial information, we can evaluate Eq. 71 at any given point $\left(\tau, \vec{x}_{\perp}, \eta_{s}\right)$ for the particle's probability distribution in momentum space. To sample the particle's transverse momentum $\left(p_{T}, \phi_{p}\right)$ from this distribution we use the the direct PDF method. In the end, since we assume longitudinal boost-invariance, the particle's rapidity can be sampled uniformly within given rapidity range. By sampling particles in this order, we optimize the sampling of the particle's spatial coordinates since the inverse CDF method has zero rejection rate. The direct PDF method used in momentum space, on the other hand, allows us to use continuous random variables for $p_{\perp}$ and $\phi_{p}$ instead of sampling them at some discrete lattice points.

A second way to proceed is to first sample the particle's momentum information with the inverse CDF method. To this end we first build the inverse CDF for the particle's momentum variables, $\left(p_{\perp}, \phi_{p}\right)$. Using 15 points in $p_{\perp}$ and 48 points in $\phi_{p}$. Once we have $\left(p_{\perp}, \phi_{p}\right)$, Eq. (71p) is used as a probability distribution for the particle's spatial coordinates $\left(\tau, \vec{x}_{\perp}, \eta_{s}\right)$ which is then sampled with the direct PDF method.

\subsubsection{Semi-analytic approach}

In a given collision event the number of particles of species $a$ being emitted from a given fluid cell at $x^{\mu}$ can be calculated analytically as follows:

$$
\Delta N_{a}\left(\tau_{f}, \vec{x}_{\perp}, \eta_{s}\right)=\frac{g_{a}}{(2 \pi)^{3}} \Delta^{3} \sigma_{\mu} \int \frac{d^{3} p}{E} p^{\mu}\left(f_{0}(p)+\delta f(p)\right) .
$$

Here the surface element of the given fluid cell is $\Delta^{3} \sigma_{\mu}=\sigma_{\mu} \Delta^{2} x_{\perp} \tau \Delta \eta_{s}$ with $\sigma_{\mu}=\left(\cosh \eta_{s}\right.$, $-\partial \tau / \partial x,-\partial \tau / \partial y,-\sinh \eta_{s}$ ). The off-equilibrium correction $\delta f$ originating from the shear stress tensor does not contribute to the total particle yield, due to the properties that $\pi^{\mu v}$ is traceless and orthogonal to the flow velocity.

$$
\int \frac{d^{3} p}{E} p^{\mu} \delta f(p)=\int \frac{d^{3} p}{E} p^{\mu} f_{0}(p)\left(1 \pm f_{0}(p)\right) \frac{\pi_{\alpha \beta} \hat{p}^{\alpha} \hat{p}^{\beta}}{2(e+P)} \chi\left(\frac{p}{T}\right)=A u^{\mu},
$$


where $A=u_{\mu} \int \frac{d^{3} p}{E} p^{\mu} \delta f(p)=\frac{\pi_{\alpha \beta}}{2(e+P)} \int \frac{d^{3} p}{E}(u \cdot p) \frac{p^{\alpha} p^{\beta}}{(u \cdot p)^{2}} \chi\left(\frac{p}{T}\right) f_{0}(p)\left(1 \pm f_{0}(p)\right)$. In the local rest frame of the fluid cell, it is easy to see that the integrand is proportional to $\delta^{\alpha \beta}$, hence

$$
A=\int d^{3} p f_{0}(p)\left(1 \pm f_{0}(p)\right) \frac{p^{2}}{3 E^{2}} \frac{\pi_{\alpha}^{\alpha}}{2(e+P)} \chi\left(\frac{p}{T}\right)=0 .
$$

Thus the particle yield is totally determined by its equilibrium distribution,

$$
\begin{aligned}
\Delta N_{a}\left(\tau_{f}, \vec{x}_{\perp}, \eta_{s}\right) & =\frac{g_{a}}{(2 \pi)^{3}} \Delta^{3} \sigma_{\mu} \int \frac{d^{3} p}{E} p^{\mu} f_{0}(p) \\
& =\frac{g_{a}}{(2 \pi)^{3}} \Delta^{3} \sigma_{\mu} u^{\mu} \int p^{2} d p d \phi d \cos \theta \frac{1}{e^{\beta\left(E-\mu_{a}\right)} \pm 1} \\
& =\frac{g_{a}}{2 \pi^{2}} \Delta^{3} \sigma_{\mu} u^{\mu} \frac{m_{a}^{2}}{\beta} \sum_{n=1}^{\infty} \frac{(\mp 1)^{n-1}}{n} e^{n \beta \mu_{a}} K_{2}\left(n \beta m_{a}\right) .
\end{aligned}
$$

With the assumption of boost invariance, the particle's rapidity $y$ and its space-time rapidity $\eta_{s}$ only enters in the combination $y-\eta_{s}$, and therefore $\frac{d N}{d \eta_{s}}=\frac{d N}{d y}$. This leads to the following relation:

$$
\begin{aligned}
\Delta N_{a}\left(\tau_{f}, \vec{x}_{\perp}, \eta_{s}\right) & =\frac{g_{a}}{(2 \pi)^{3}} \Delta^{3} \sigma_{\mu} \int d y \int d^{2} p_{\perp} p^{\mu} f_{0}(p) \\
& =\frac{g_{a}}{(2 \pi)^{3}} \int d y \Delta^{2} x_{\perp} \tau \Delta \eta_{s} \int d^{2} p_{\perp}\left(m_{\perp} \cosh \left(y-\eta_{s}\right)-\vec{p}_{\perp} \cdot \vec{\nabla}_{\perp} \tau\right) f_{0}(p) \\
& =\Delta \eta_{s} \frac{g_{a}}{(2 \pi)^{3}} \int \tau d \tilde{y} \Delta^{2} x_{\perp} \int d^{2} p_{\perp}\left(m_{\perp} \cosh (\tilde{y})-\vec{p}_{\perp} \cdot \vec{\nabla}_{\perp} \tau\right) f_{0}(p)
\end{aligned}
$$

This integral is independent of $\eta_{s}$, so

$$
\Delta N_{a}\left(\tau_{f}, \vec{x}_{\perp}, \eta_{s}\right)=\Delta \eta_{s} \frac{\Delta N}{\Delta y}\left(\tau, \vec{x}_{\perp}\right) .
$$

In the numerical sampling procedure, we first consider all freeze-out fluid cells $\left(\tau, \vec{x}_{\perp}\right)$ in the transverse plane and use (80) (together with (82)) to compute the total particle yield per unit rapidity for particle species $a, \Delta N / \Delta y$, for each cell. If freeze-out occurs on a surface of constant inverse temperature $\beta$ and chemical potential $\mu_{a}$, as will be the assumed in the rest of this thesis, Eq. 80, can be written as,

$$
\Delta N_{a}\left(\tau_{f}, \vec{x}_{\perp}, \eta_{s}\right)=n_{a} u_{\mu}\left(\tau_{f}, \vec{x}_{\perp}, \eta_{s}\right) \Delta^{3} \sigma_{\mu}\left(\tau_{f}, \vec{x}_{\perp}, \eta_{s}\right) .
$$

where $n_{a}=\frac{g_{a}}{2 \pi^{2}} \frac{m_{a}^{2}}{\beta} \sum_{n=1}^{\infty} e^{n \beta \mu_{a}} K_{2}\left(n \beta m_{a}\right)$ is the freeze-out density of particle species $a$, which is the same for all freeze-out cells. In this case, $\Delta N_{a} / \Delta y$ depends on the position of the fluid cell only through its freeze-out volume,

$$
\Delta V\left(\tau_{f}, \vec{x}_{\perp}, \eta_{s}\right)=u^{\mu} \Delta^{3} \sigma_{\mu}\left(\tau_{f}, \vec{x}_{\perp}, \eta_{s}\right) .
$$

The we use $\left(\Delta N_{a} / \Delta y\right)\left(\tau_{f}, \vec{x}_{\perp}\right)$ to build up an inverse CDF for the spatial variables $\left(\tau_{f}, \vec{x}_{\perp}\right)$. Their sum over all $\left(\tau_{f}, \vec{x}_{\perp}\right)$ points gives the total rapidity density $\frac{d N_{a}}{d y}$ of particle species $a$ in a given collision event. The constructed inverse CDF is then used to sample the positions $\left(\tau_{f}, \vec{x}_{\perp}\right)$ of 
the particles of species $a$. Finally, we use the Cooper-Frye formula Eq. 71] at these sampled positions $\left(\tau_{f}, \vec{x}_{\perp}\right)$ as the relative probability distribution for sampling the particle's momentum $\left(p_{T}, \phi_{p}, y-\eta_{s}\right)$ using the direct PDF method:

$$
\begin{aligned}
P\left(p_{\perp}, \phi_{p}, y-\eta_{s} ; \tau_{f}, \vec{x}_{\perp}\right)= & \frac{g_{i}}{(2 \pi)^{3}} \Delta^{3} \sigma_{\mu} p^{\mu}\left(f_{0}(p)+\delta f(p)\right) \\
= & \frac{g_{i}}{(2 \pi)^{3}} \tau_{f} \Delta^{2} x_{\perp} \Delta \eta_{s}\left(m_{\perp} \cosh \left(y-\eta_{s}\right)-\vec{p}_{\perp} \cdot \vec{\nabla}_{\perp} \tau\right) \\
& \times\left(f_{0}(p)+\delta f(p)\right) .
\end{aligned}
$$

Having obtained $\left(y-\eta_{s}\right)$ by sampling Eq. $[85$, we use boost-invariance and sample $y$ uniformly from a given range specified by the user (e.g., -4 to 4 ) and then obtain $\eta_{s}$ from the previously determined $y-\eta_{s}$.

Since for every $\left(\tau, \vec{x}_{\perp}\right)$, the probability Eq. 85 for $\left(p_{T}, \phi_{p}, y-\eta_{s}\right)$ is only sampled once, building an inverse CDF for Eq. (85) would be excessively expensive, which is why we choose to use the direct PDF method to sample $\left(p_{\perp}, \phi_{p}, y-\eta_{s}\right)$. However, the direct PDF method requires one to estimate the maximum value of the probability distribution function given in Eq. 85 which is closely related to the function

$$
G(E ; A)=\frac{E^{A}}{e^{\beta(E-\mu)} \pm 1}, A>0 .
$$

By setting its derivative to zero, the extrema can be found by solving

$$
\left(1 \mp f_{0}\right)=\frac{A}{\beta E} \Longleftrightarrow\left\{\begin{array}{l}
x e^{x}=y ; x=\beta E-A, y=A e^{\beta \mu-A}, \text { fermions (upper) }, \\
x e^{-x}=y ; x=A-\beta E, y=A e^{\beta \mu-A}, \text { bosons (lower) }
\end{array}\right.
$$

This equation is transcendental and cannot be solved algebraically; however, the solutions to the equations $x e^{ \pm x}=y$ in Eq. 87) can be pre-calculated and tabulated. For fermions (upper sign), a solution always exists and it is expressed by the Lambert W-function; for bosons (lower sign) the equation has real solutions only when $y<1 / e$, and the it yields two solutions; the physical solution must satisfy $x \in[0,1]$. In the following, the solution to Eq. (87) will be denoted as $E_{\max }^{ \pm}$ when it exists.

The maximum of $G(E ; A)$ with constraint $E \geq m$ will be denoted as $G_{\max }^{(A)}$. It depends on several conditions:

1. For fermions (upper sign), $G(E)$ has a single peak at $E_{\max }^{+}$and the constraint maximum is taken as $G\left(E_{\max }^{+}\right)$if $E_{\max }^{+}>m$ and as $G(m)$ otherwise.

2. For bosons (lower sign) with $A e^{\beta \mu-A}>1 / e$, Eq. 87 has no solution and the maximum takes $G(m)$.

3. For bosons (lower sign) with $A e^{\beta \mu-A} \leq 1 / e, G(E)$ has two extrema in $(\mu, \infty)$, with the larger one being the maximum and given by $E_{\max }^{-}$. If $E_{\max }^{-}<m$ then the maximum is taken as $G(m)$; otherwise the maximum is taken as the larger one of the two numbers $G(m)$ and $G\left(E_{\max }^{-}\right)$.

In Eq. 85, an upper limit for the factor $p^{\mu} \Delta^{3} \sigma_{\mu}$ can obtained using the Hölder inequality,

$$
p^{\mu} \Delta^{3} \sigma_{\mu}=E \Delta^{3} \sigma_{0}+p^{i} \Delta \sigma_{i} \leq(p \cdot u)\left(\left|\Delta^{3} \sigma_{\mu} u^{\mu}\right|+\sqrt{\left|\Delta^{3} \sigma_{\mu} \Delta^{3} \sigma_{v} \Delta^{\mu \nu}\right|}\right) .
$$


For the equilibrium contribution, it is clear that the remaining part is to calculate the maximum of the function

$$
E f_{0}=\frac{E}{e^{(E-\mu) / T} \pm 1}=G(E ; 1)
$$

the solution to this problem is $G_{\max }^{(1)}$.

For the off-equilibrium correction, it is convenient to estimate its maximum in the local rest frame of the fluid cell. We can further rotate the shear stress tensor in the transverse plane such that $\pi^{x y}=0$. In such a coordinate system

$$
\begin{aligned}
p^{\mu} p^{v} \pi_{\mu \nu} & =\left(p^{x}\right)^{2} \pi_{x x}+\left(p^{y}\right)^{2} \pi_{y y}+\left(p^{z}\right)^{2} \pi_{z z} \leq E\left(\left|p^{x} \pi_{x x}\right|+\left|p^{y} \pi_{y y}\right|+\left|p^{z} \pi_{z z}\right|\right) \\
& \leq E^{2} \sqrt{\pi_{x x}^{2}+\pi_{y y}^{2}+\pi_{z z}^{2}}=E^{2} \sqrt{\pi^{\mu \nu} \pi_{\mu \nu}} .
\end{aligned}
$$

In the last step, we rewrote the expression again in Lorentz invariant form such that it is now valid in any frame. With the form of $\delta f$ in Eq. (72) and assuming $f_{0}<1$,

$$
E^{\alpha+1} f_{0}\left(1 \mp f_{0}\right) \leq \lambda E^{\alpha+1} f_{0}=\lambda G(E ; \alpha+1) \leq \lambda G_{\max }^{(\alpha+1)},
$$

where $\lambda=1$ for fermions and $\lambda=2$ for bosons. To summarize, the maximum of the PDF Eq. 85 for $\left(p_{\perp}, \phi_{p}, y-\eta_{s}\right)$ can be estimated as

$$
P \leq P_{\max }=\frac{g_{a}}{(2 \pi)^{3}} \tau\left(\left|\Delta^{3} \sigma_{\mu} u^{\mu}\right|+\sqrt{\Delta^{3} \sigma_{\mu} \Delta^{3} \sigma_{v} \Delta^{\mu \nu}}\right)\left(G_{\max }^{(1)}+\frac{\sqrt{\pi^{\mu v} \pi_{\mu \nu}}}{2(e+P) T^{\alpha}} \lambda G_{\max }^{(\alpha+1)}\right) .
$$

For light mesons, the validity of the assumption $f_{0}<1$ depends on the value of the freeze-out temperature and chemical potential. Especially, kinetic freeze-out at temperature much below the chemical decoupling temperature can lead to large non-equilibrium chemical potentials that can cause this assumption to break down in some of the fluid cells. We found that $f_{0}<1$ almost all the time, although there were some instances where it was violated. If a more rigorous result is desired, the inequality (91) can be replaced by the following one:

$$
E^{\alpha+1} f_{0}\left(1+f_{0}\right) \leq\left|E^{\alpha+1} f_{0}\right|+\left|E^{\gamma} f_{0}\right|\left|E^{\alpha+1-\gamma} f_{0}\right| \leq G_{\max }^{(\alpha+1)}+G_{\max }^{(\gamma)} G_{\max }^{(\alpha+1-\gamma)},
$$

where $0 \leq \gamma \leq \alpha+1$.

\subsubsection{The negative probability issue}

For hyper-surface of constant temperature, the Cooper-Frye formula in Eq. (71) is not positive semi-definite. This is because on an isothermal hyper-surface $\Sigma, d^{3} \sigma_{\mu}$ can be a space-like vector. So $p^{\mu} d^{3} \sigma_{\mu}$ can be negative in certain regions. Physically, such regions represent parts of the switching surface through which more particles are flying into the fireball instead of being emitted. These negative contributions to the Cooper-Frye integral are essential to ensure the conservation of energy across the hyper-surface. However, they become problematic when one wants to use Eq. (71) as a probability distribution (which should always be positive). In the practical sampling procedure, we insert a $\theta$-function by hand to enforce positivity of the probability distribution function. Since we group the random variables differently in the different sampling approaches discussed above, insertion of the $\theta$-function will be done slightly differently in each case, with different consequences. In each case, a slight violation of energy-momentum conservation will occur. Let us therefore explore the implications of the $\theta$-function in some detail, 
we first sample particle's spatial information using the purely numerical approach, we use a $\theta$ function $\theta\left(u^{\mu} d \sigma_{\mu}\right)$ to enforce positivity of the $p_{\perp}$-integrated distribution function. This means that none of our sampled particles will come from the spatial regions where $u^{\mu} d \sigma_{\mu}<0$. In the second step, when sampling the momenta we enforce the positivity of Eq. (71) at the already sampled spatial coordinates. In this step, there are two possible quantities that can become negative. First, $p^{\mu} \sigma_{\mu}$ may be negative for some values of $p^{\mu}$. This represents the situation where a net number of particles with momentum $p^{\mu}$ flies into the fireball. Secondly, in the viscous case, when the off-equilibrium correction $\delta f$ becomes large, it may overwhelm the equilibrium term and turn the entire distribution function to negative. This situation represents a breakdown of the Chapman-Enskog expansion keeping only terms of first order in $\delta f$, Eq. (71) should not be trusted in such regions of momentum space. With $\eta / s=0.20$, we find that this problem usually occurs at high $p_{T}>2.5 \mathrm{GeV}$. In the sampling procedure, we enforce both terms to be always positive, by inserting a product of theta functions, $\theta\left(f_{0}+\delta f\right) \theta\left(p^{\mu} d^{3} \sigma_{\mu}\right) . \theta\left(f_{0}+\delta f\right)$ should be always kept in the calculation, even for the analytic results. The second factor $\theta\left(p^{\mu} d \sigma_{\mu}\right)$ causes a deviation of the sampled momentum distribution from the analytical result which will be studied below.

If we first sample the particle's momentum information, we enforce positivity of the momentum distribution $d N /\left(d y p_{\perp} d p_{\perp} d \phi_{p}\right) \geq 0$. In most cases, the total number of emitted particles with given transverse momentum $\vec{p}_{\perp}$, integrated over the entire freeze-out surface, is positive. The positivity constraint on $d N /\left(d y p_{T} d p_{T} d \phi_{p}\right)$ therefore has almost no effect at all. The set of momentum configurations obtained from this sampling procedure will reproduce momentum distributions and flow coefficients that agree most closely with the analytical Cooper-Frye formalism. In the second step, when we then additionally sample particle's spatial information, we need to enforce positivity of Eq. 71 at a given momentum $\vec{p}_{\perp}$. Regions on the hypersurface where Eq. 71 is negative will thus not contribute to particle emission at that $\vec{p}_{\perp}$. The sampled spatial distribution will therefore show some deviation from the analytic result.

For the semi-analytic approach, the situation is similar to the purely numerical approach when sampling the positions first and the momenta second.

\subsubsection{Multiplicity fluctuations at freeze-out}

The Cooper-Frye formula only yields the average number of particles emitted from a given hydrodynamic event. Each sampling of the Cooper-Fyre formula will, however, result in a number of emitted particle that fluctuates around that mean value. In principle, these sampling fluctuations are constrained by energy-momentum, baryon number and charge conservation. However, exact implementation of these constraints is non-trivial and will have to be left for future studies.

We use an approximation based on the following procedure: We compute the integer value of the number of particles of species as predicted by Cooper-Frye, sample such particles until that number is exhausted, and then use the non-integer part of the predicted number to uniformly sample for one additional particle. This sampling procedure introduces minimum fluctuations in the total number of particles. In the current version of iSS, there are options for users to instead fluctuate the particle number according to Poisson or negative Binomial distributions.

\subsubsection{Performance}

To demonstrate the performance of the iSS algorithm, we use an event-averaged hydrodynamically evolved $\mathrm{Pb}+\mathrm{Pb}$ profile at $20-30 \%$ centrality at LHC energy to obtain a rough estimate for the average running time of our particle sampler. The two sampling approaches have their individual advantages and disadvantages in dealing with different sampling requirements. 


\begin{tabular}{|c|c|c|}
\hline 100 repeated samplings & purly numerical approach & semi-analytic approach \\
\hline $\begin{array}{l}\text { determining particle } \\
\text { yield } d N / d y\end{array}$ & $\begin{array}{c}21.84 \mathrm{~s} \text { for } \pi^{+} \\
\sim \times 100 \text { for rest of particles }\end{array}$ & $\begin{array}{c}\text { negligible for } \pi^{+} \\
0.01 \mathrm{~s} \text { for all particles }\end{array}$ \\
\hline actual sampling & $\begin{array}{c}1.25 \mathrm{~s} \text { for } \pi^{0} \\
\text { faster for heavier particles }\end{array}$ & $\begin{array}{l}2.65 \mathrm{~s} \text { for } \pi^{+} \\
\text {faster for heavier particles }\end{array}$ \\
\hline total & $2463.75 \mathrm{~s}$ & $15.16 \mathrm{~s}$ \\
\hline $\begin{array}{c}50,000 \text { sampling } \\
\left(\pi^{+} \text {only }\right)\end{array}$ & $88.73 \mathrm{~s}$ & $1327.99 \mathrm{~s}$ \\
\hline
\end{tabular}

Table 6: Efficiency comparison between pure numerical and semi-analytic methods. The test case has 32869 conversion surface cells in the transverse plane and $d N /\left.d y\right|_{\pi^{+}} \sim 144$. The test is done on a single core personal laptop.

The numerical performance of the code is summarized in Table. 6. The tests are done with the Intel $\mathrm{C}++$ compiler with -03 optimization. Our code runs about a factor of 6 faster with the Intel compiler compared to the GNU compiler $(\mathrm{g}++)$.

The purely numerical approach is most suitable when a large number of repeated samplings of a single hydrodynamical event is desired. This is essential if one wants to study with good statistical precision rare multi-strange hadrons very few of which are emitted in a single event. On the other hand, the semi-analytic approach is extremely fast for small numbers of repeated samplings. This large gain in the numerical efficiency is due to the fact that it determines the particle yields analytically using, Eq. 80, The drawback is that in this approach we need to sample one additional dimension (the rapidity direction) using the direct PDF method, which reduces the total sampling efficiency per simulation cycle.

\subsection{Code verification}

In this section, we show some test results from our particle sampler.
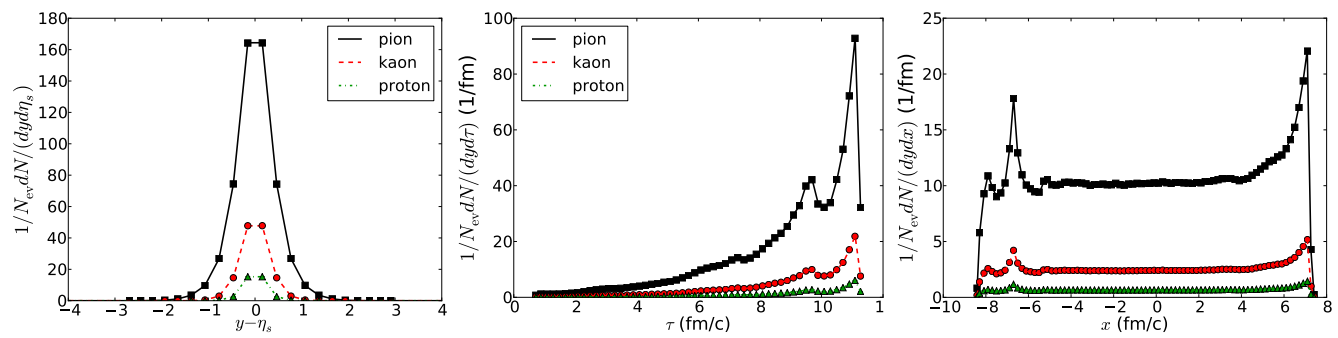

Figure 15: For sampling method $\mathrm{I}$ in the purely numerical approach, the spatial distributions of the sampled thermal particles (solid dots) are compared to the emission function calculated from the Cooper-Frye Formula (lines). The left panel shows the particle distribution along the $\eta_{s}$ direction. The middle panel is the time emission function of the particles and the right panel shows the particle distribution along the $\mathrm{x}$-axis in the transverse plane. All results are from a single hydrodynamic event with bumpy initial conditions.

In Figs. 15 and 16 we present the spatial and momentum distributions of thermally emitted particles (pions, kaons, and protons) and compare them against the emission function calculated directly from the Cooper-Frye Formula. We perform repeated samplings for a single hydrodynamic simulation with fluctuating initial conditions which emits about 172 positive pions, 40 

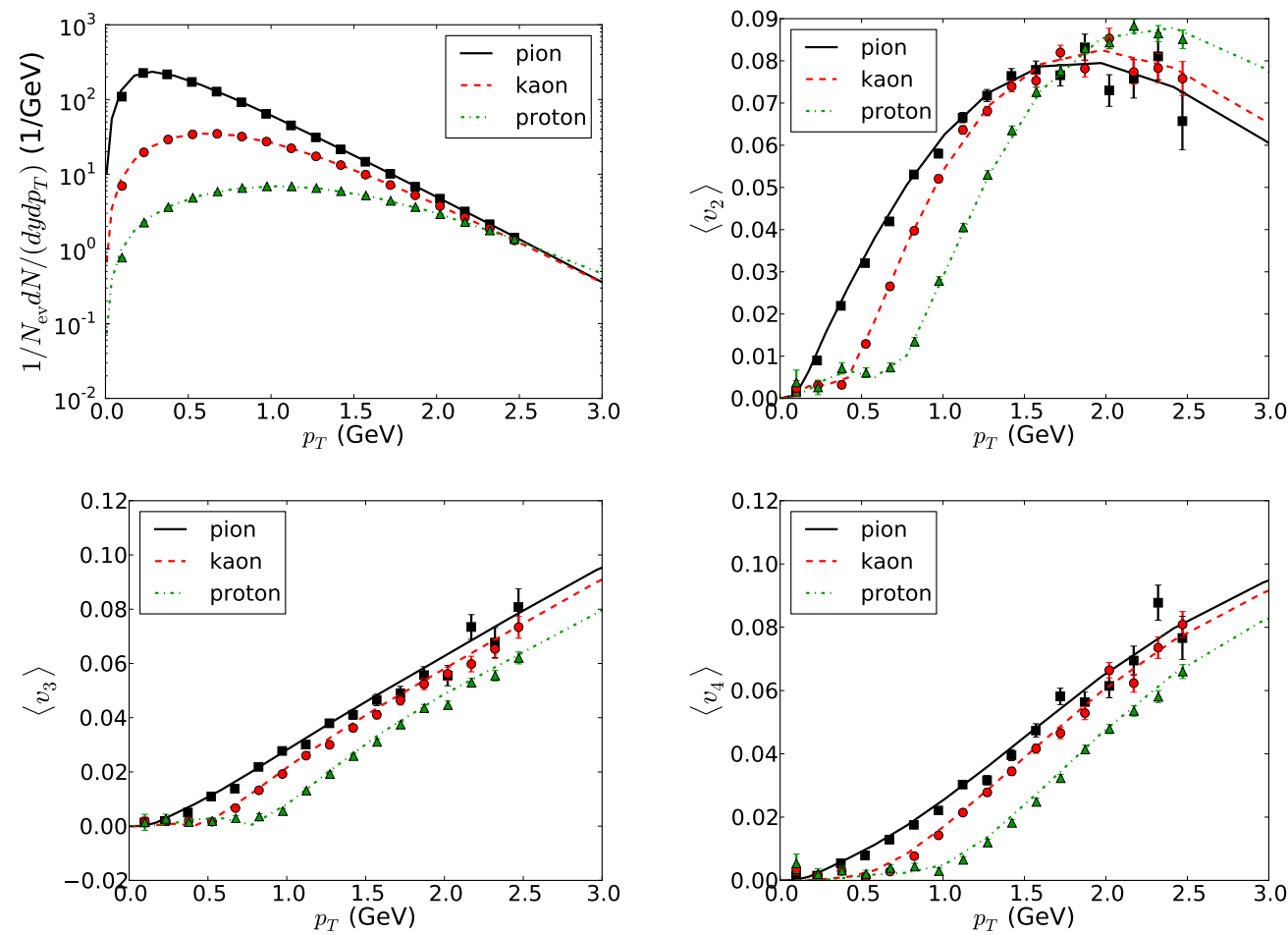

Figure 16: Similar to Fig. 15, momentum distributions of the sampled particles are compared with results from the Cooper-Frye formula. Particles' $p_{T}$-differential spectra, $v_{2}, v_{3}$, and $v_{4}$ are presented.

positive kaons, and 11 protons. per unit rapidity thermally (i.e., not counting particles from resonance decays). To obtain sufficient statistics, we sample 50,000 events for thermal pions, 150,000 events for thermal kaons, and 500,000 events for thermal protons (these numbers account for the relative yields per event of their particle species.) Samples are generated using the purely numerical approach method I, which samples the spatial distributions first and then particle momenta.

We find that the particle samples generated from this method reproduce very accurately the spatial distributions from the Cooper-Frye Formula. The regions where $u_{\mu} d^{3} \sigma^{\mu}<0$ do not affect the partially integrated emission functions shown in Fig. 15. In Fig. 16 , we compare the particle momentum distributions against the results from the Cooper-Frye formula. We find very good agreement for the particle spectra as well as for the their anisotropy coefficients $v_{2}, v_{3}$, and $v_{4}$.

In Figs. 17 and 18, we show similar comparisons using the semi-analytic approach. Again the spatial distributions in the transverse plane from directly integrating the Cooper-Frye formula are very well reproduced but some slight differences are seen in the $\eta_{s}$ and $\tau$ distributions. Fig. 18 shows that this method generates some noticeable disagreement in the higher order momentum anisotropies of the particle momentum distribution, caused by the particular way we remove negative contributions in this approach.

Finally, we shown in Figs. 19 and 20, our results from the sampling method II within the purely numerical approach. In this method, since we first sample the momentum distributions of 

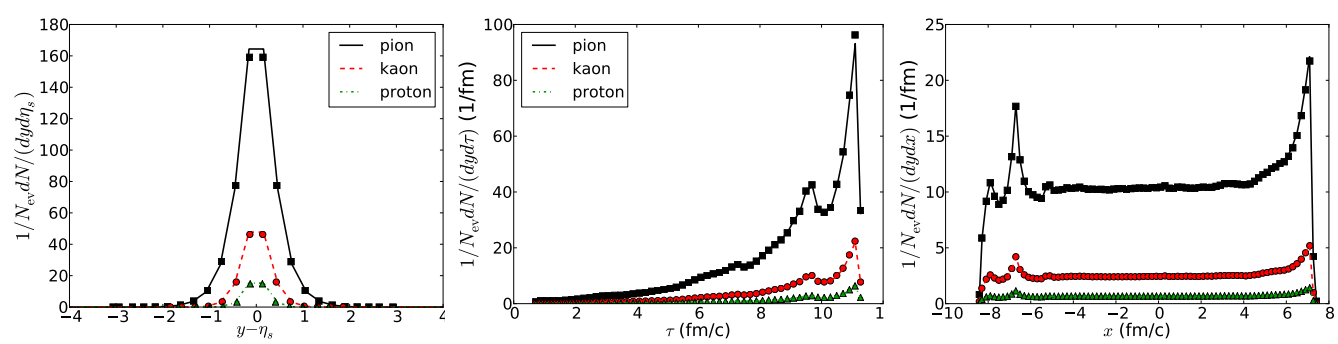

Figure 17: Similar to Fig. 15 but for sampling method with the semi-analytic approach.
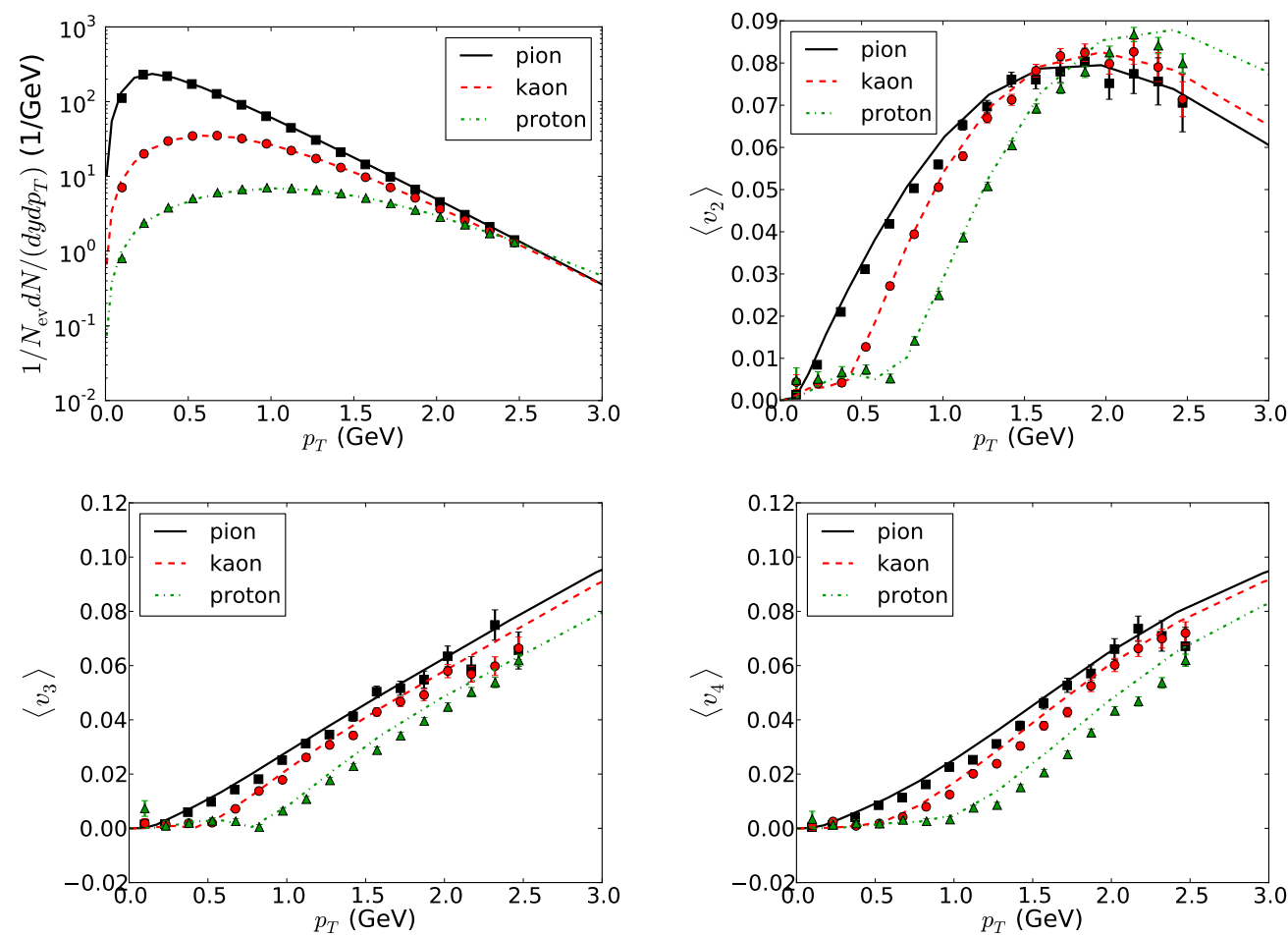

Figure 18: Similar to Fig. 16 but for sampling with the semi-analytic approach.

the particles, the sampled results reproduce the directly calculated particle momentum distributions from the Cooper-Frye formula very well. The spatial distributions of the particles, on the other hand, exhibit some noticeable deviations from the Cooper-Frye results, due to the removal of negative contributions.

\section{OSCAR to URQMD: osc $2 u$}

The ISS module produces an ensemble of hadrons on the particalization hyper surface each hadron being characterized by its production time and location, i.e. its position on the 

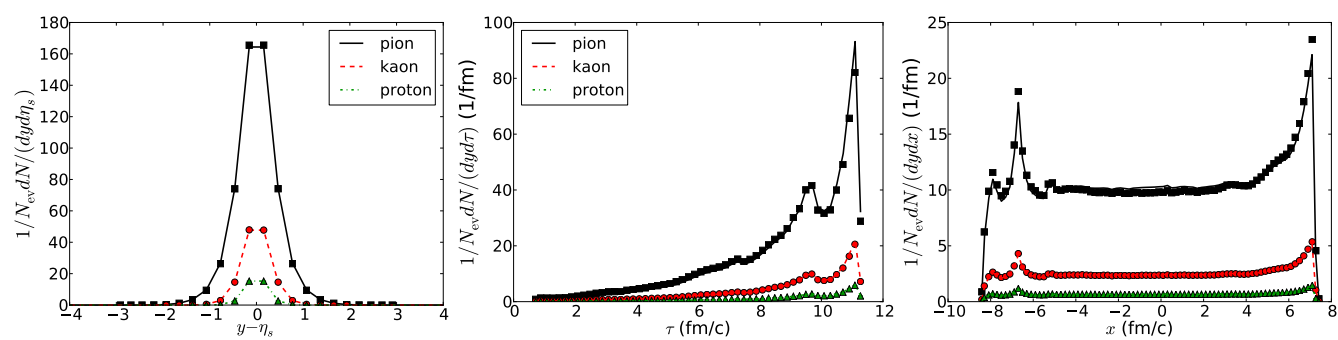

Figure 19: Similar to Fig. 15. but for sampling method II of the purely numerical approach, which samples particle momenta first.
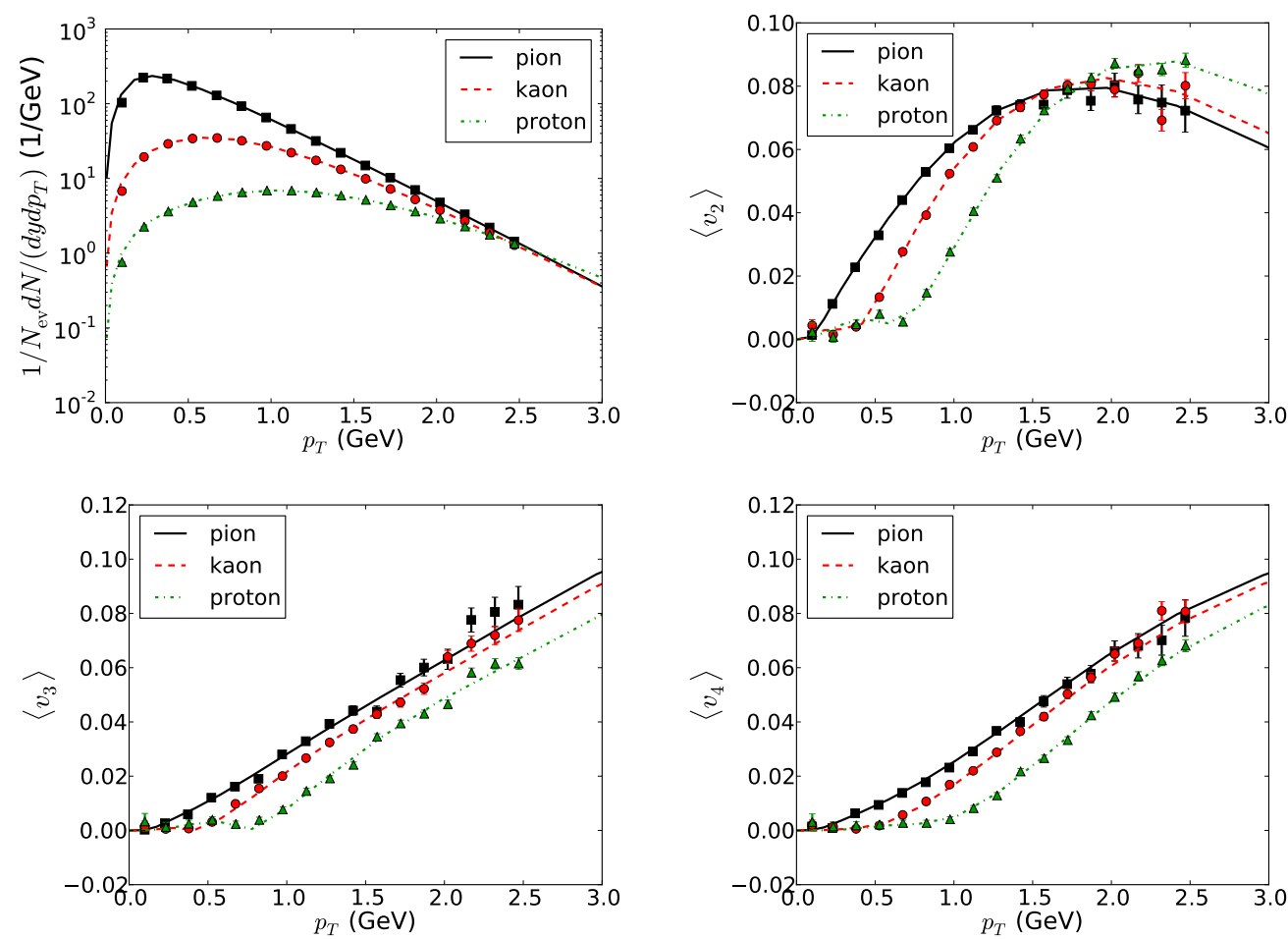

Figure 20: Similar to Fig. 16 but for sampling method II of the purely numerical approach, which samples particle momenta first.

hyper surface, as well as its momentum, flavor-type, mass and quantum numbers. This ensemble is written out as a particle list in the standard OSCAR1997A format $7^{7}$ When running the code package in hybrid (VISHNU) mode, this list needs to be converted into an initial condition file for the hadronic rescattering model URQMD, which is the function of the Oscar to UrQMD converter osc2u.

Apart from reformatting the ISS output to the UrQMD initial condition format, osc $2 u$ fulfills

https://karman.physics.purdue.edu/OSCAR-old/docs/file/cascade_output_format/node8.html 
another important function, namely to synchronize all particles in the ensemble to the UrQMD computational frame: in the ISS output every particle is given with the time of its creation on the hyper surface. UrQMD, however, requires all particles to be in the same computational frame in order to solve the Boltzmann collision integral. To achieve this, osc2u propagates all hadrons backwards in time from their production time $\tau_{f}$ to $t=0$ and assigns each hadron $\tau_{f}$ as formation time. The UrQMD calculation thus starts with an initial condition at time $t=0$ in its computational frame, propagating forward in time. During their formation time, hadrons are assigned zero cross section and do not interact. Therefore all hadrons in the initial condition will first start interacting at the location and time of their actual creation on the hyper surface.

\section{Hadronic Rescattering: UrQMD}

Ultra-relativistic Quantum Molecular Dynamics (UrQMD) is a microscopic transport model ideally suited for the description of the dynamics of a system of hadrons, both in and out of equilibrium [18, 19]. The UrQMD approach is in spirit closely related to hadronic cascade [63], Vlasov-Uehling-Uhlenbeck [64] and (R)QMD transport models [65, 66] and has been extensively used to model the evolution of hadronic systems in a variety of settings, from a wide range of heavy-ion collisions to cosmic ray showers.

We shall describe here only the part of the model that is important for the application at hand, namely the evolution of an expanding hadron gas initially in local equilibrium (but subsequently not anymore constrained by any equilibrium assumptions), starting at the particalization or switching temperature $T_{s w}$. The treatment of high-energy hadron-hadron scatterings, as it occurs in the initial stage of ultrarelativistic collisions, is not discussed here. A complete description of the model and detailed comparisons to experimental data can be found in [18, 19].

The UrQMD modeling package solves a Boltzmann equation for the distribution function of all hadrons in the system by evolving the system of hadrons through a sequence of binary collisions or $2-N$-body decays.

Binary collisions are performed in a point-particle sense: Two particles collide if their minimum distance $d$, i.e. the minimum relative distance of the centroids of the Gaussians during their motion, in their CM frame fulfills the requirement:

$$
d \leq d_{0}=\sqrt{\frac{\sigma_{\text {tot }}}{\pi}}, \quad \sigma_{\text {tot }}=\sigma(\sqrt{s}, \text { type }) .
$$

The cross section is assumed to be the free cross section of the regarded collision type $(N-N$, $N-\Delta, \pi-N, \pi-\pi, \ldots)$.

The UrQMD collision term contains 53 different baryon species (including nucleon, delta and hyperon resonances with masses up to $2 \mathrm{GeV}$ ) and 24 different meson species (including strange meson resonances), which are supplemented by their corresponding anti-particle and all isospinprojected states. The baryons and baryon-resonances which can be populated in UrQMD are listed in table 7, the respective mesons in table 8- full baryon/antibaryon symmetry is included (not shown in the table), both, with respect to the included hadronic states, as well as with respect to the reaction cross sections. All hadronic states can be produced in string decays, s-channel collisions or resonance decays (string excitations and decays do not play a significant role for the hadron gas evolution discussed here).

Tabulated and parameterized experimental cross sections are used when available. Resonance absorption, decays and scattering are handled via the principle of detailed balance. If no 


\begin{tabular}{cccccc}
\hline \hline nucleon & delta & lambda & sigma & xi & omega \\
\hline \hline$N_{938}$ & $\Delta_{1232}$ & $\Lambda_{1116}$ & $\Sigma_{1192}$ & $\Xi_{1317}$ & $\Omega_{1672}$ \\
$N_{1440}$ & $\Delta_{1600}$ & $\Lambda_{1405}$ & $\Sigma_{1385}$ & $\Xi_{1530}$ & \\
$N_{1520}$ & $\Delta_{1620}$ & $\Lambda_{1520}$ & $\Sigma_{1660}$ & $\Xi_{1690}$ & \\
$N_{1535}$ & $\Delta_{1700}$ & $\Lambda_{1600}$ & $\Sigma_{1670}$ & $\Xi_{1820}$ & \\
$N_{1650}$ & $\Delta_{1900}$ & $\Lambda_{1670}$ & $\Sigma_{1775}$ & $\Xi_{1950}$ & \\
$N_{1675}$ & $\Delta_{1905}$ & $\Lambda_{1690}$ & $\Sigma_{1790}$ & & \\
$N_{1680}$ & $\Delta_{1910}$ & $\Lambda_{1800}$ & $\Sigma_{1915}$ & & \\
$N_{1700}$ & $\Delta_{1920}$ & $\Lambda_{1810}$ & $\Sigma_{1940}$ & & \\
$N_{1710}$ & $\Delta_{1930}$ & $\Lambda_{1820}$ & $\Sigma_{2030}$ & & \\
$N_{1720}$ & $\Delta_{1950}$ & $\Lambda_{1830}$ & & & \\
$N_{1900}$ & & $\Lambda_{2100}$ & & & \\
$N_{1990}$ & & $\Lambda_{2110}$ & & & \\
$N_{2080}$ & & & & & \\
$N_{2190}$ & & & & & \\
$N_{2200}$ & & & & & \\
$N_{2250}$ & & & & & \\
\hline \hline
\end{tabular}

Table 7: Baryons and baryon-resonances treated in UrQMD. The corresponding antibaryon states are included as well.

\begin{tabular}{cccccc}
\hline \hline $0^{-}$ & $1^{-}$ & $0^{+}$ & $1^{+}$ & $2^{+}$ & $\left(1^{-}\right)^{*}$ \\
\hline \hline$\pi$ & $\rho$ & $a_{0}$ & $a_{1}$ & $a_{2}$ & $\rho(1450)$ \\
$K$ & $K^{*}$ & $K_{0}^{*}$ & $K_{1}^{*}$ & $K_{2}^{*}$ & $\rho(1700)$ \\
$\eta$ & $\omega$ & $f_{0}$ & $f_{1}$ & $f_{2}$ & $\omega(1420)$ \\
$\eta^{\prime}$ & $\phi$ & $f_{0}^{*}$ & $f_{1}^{\prime}$ & $f_{2}^{\prime}$ & $\omega(1600)$ \\
\hline \hline
\end{tabular}

Table 8: Mesons and meson-resonances, sorted with respect to spin and parity, treated in UrQMD. 
experimental information is available, the cross section is either calculated via an One-BosonExchange (OBE) model or via a modified additive quark model which takes basic phase space properties into account.

In the baryon-baryon sector, the total and elastic proton-proton and proton-neutron cross sections are well known [67]. Since their functional dependence on $\sqrt{s_{\mathrm{NN}}}$ shows a complicated shape at low energies, UrQMD uses a table-lookup for those cross sections. However, many cross sections involving strange baryons and/or resonances are not well known or even experimentally accessible - for these cross sections the additive quark model is widely used.

At RHIC and LHC energies, the most important reaction channels are meson-meson and meson-baryon elastic scattering and resonance formation. For example, the total meson-baryon cross section for non-strange particles is given by

$$
\begin{aligned}
\sigma_{\text {tot }}^{M B}(\sqrt{s})= & \sum_{R=\Delta, N^{*}}\left\langle j_{B}, m_{B}, j_{M}, m_{M} \| J_{R}, M_{R}\right\rangle \\
& \times \frac{2 S_{R}+1}{\left(2 S_{B}+1\right)\left(2 S_{M}+1\right)} \times \frac{\pi}{p_{C M S}^{2}} \frac{\Gamma_{R \rightarrow M B} \Gamma_{t o t}}{\left(M_{R}-\sqrt{s}\right)^{2}+\frac{\Gamma_{t o t}^{2}}{4}}
\end{aligned}
$$

with the total and partial $\sqrt{s}$-dependent decay widths $\Gamma_{t o t}$ and $\Gamma_{R \rightarrow M B}$. The full decay width $\Gamma_{t o t}(M)$ of a resonance is defined as the sum of all partial decay widths and depends on the mass of the excited resonance:

$$
\Gamma_{t o t}(M)=\sum_{b r=\{i, j\}}^{N_{b r}} \Gamma_{i, j}(M)
$$

The partial decay widths $\Gamma_{i, j}(M)$ for the decay into the final state with particles $i$ and $j$ is given by

$$
\Gamma_{i, j}(M)=\Gamma_{R}^{i, j} \frac{M_{R}}{M}\left(\frac{\left\langle p_{i, j}(M)\right\rangle}{\left\langle p_{i, j}\left(M_{R}\right)\right\rangle}\right)^{2 l+1} \times \frac{1.2}{1+0.2\left(\frac{\left\langle p_{i, j}(M)\right\rangle}{\left\langle p_{i, j}\left(M_{R}\right)\right\rangle}\right)^{2 l}},
$$

here $M_{R}$ denotes the pole mass of the resonance, $\Gamma_{R}^{i, j}$ its partial decay width into the channel $i$ and $j$ at the pole and $l$ the decay angular momentum of the final state. All pole masses and partial decay widths at the pole are taken from the Review of Particle Properties [67]. $\Gamma_{i, j}(M)$ is constructed in such a way that $\Gamma_{i, j}\left(M_{R}\right)=\Gamma_{R}^{i, j}$ is fulfilled at the pole. In many cases only crude estimates for $\Gamma_{R}^{i, j}$ are given in [67] - the partial decay widths must then be fixed by studying exclusive particle production in elementary proton-proton and pion-proton reactions. Therefore, e.g., the total pion-nucleon cross section depends on the pole masses, widths and branching ratios of all $N^{*}$ and $\Delta^{*}$ resonances listed in table 7. Resonant meson-meson scattering (e.g. $\pi+\pi \rightarrow \rho$ or $\left.\pi+K \rightarrow K^{*}\right)$ is treated in the same formalism.

In order to correctly treat equilibrated matter [68] (we repeat that the hadronic matter with which UrQMD is being initialized in our approach is in local chemical and thermal equilibrium), the principle of detailed balance is of great importance. Detailed balance is based on timereversal invariance of the matrix element of the reaction. It is most commonly found in textbooks in the form:

$$
\sigma_{f \rightarrow i}=\frac{\vec{p}_{i}^{2}}{\vec{p}_{f}^{2}} \frac{g_{i}}{g_{f}} \sigma_{i \rightarrow f},
$$

with $g$ denoting the spin-isospin degeneracy factors. UrQMD applies the general principle of detailed balance to the following two process classes: 
1. Resonant meson-meson and meson-baryon interactions: Each resonance created via a meson-baryon or a meson-meson annihilation may again decay into the two hadron species which originally formed it. This symmetry is only violated in the case of three- or fourbody decays and string fragmentations, since N-body collisions with $(\mathrm{N}>2)$ are not implemented in UrQMD.

2. Resonance-nucleon or resonance-resonance interactions: the excitation of baryon-resonances in UrQMD is handled via parameterized cross sections which have been fitted to data. The reverse reactions usually have not been measured - here the principle of detailed balance is applied. Inelastic baryon-resonance de-excitation is the only method in UrQMD to absorb mesons (which are bound in the resonance). Therefore the application of the detailed balance principle is of crucial importance for heavy nucleus-nucleus collisions.

Equation (98), however, is only valid in the case of stable particles with well-defined masses. Since in UrQMD detailed balance is applied to reactions involving resonances with finite lifetimes and broad mass distributions, equation (98) has to be modified accordingly. For the case of one incoming resonance the respective modified detailed balance relation has been derived in [69]. Here, we generalize this expression for up to two resonances in both, the incoming and the outgoing channels.

The differential cross section for the reaction $(1,2) \rightarrow(3,4)$ is given by:

$$
\mathrm{d} \sigma_{12}^{34}=\frac{|\mathcal{M}|^{2}}{64 \pi^{2} s} \frac{p_{34}}{p_{12}} \mathrm{~d} \Omega \prod_{i=3}^{4} \delta\left(p_{i}^{2}-M_{i}^{2}\right) \mathrm{d} p_{i}^{2},
$$

here the $p_{i}$ in the $\delta$-function denote four-momenta. The $\delta$-function ensures that the particles are on mass-shell, i.e. their masses are well-defined. If the particle, however, has a broad mass distribution, then the $\delta$-function must be substituted by the respective mass distribution (including an integration over the mass):

$$
\mathrm{d} \sigma_{12}^{34}=\frac{|\mathcal{M}|^{2}}{64 \pi^{2} s} \frac{1}{p_{12}} \mathrm{~d} \Omega \prod_{i=3}^{4} p_{34} \cdot \frac{\Gamma}{\left(m-M_{i}\right)^{2}+\Gamma^{2} / 4} \frac{\mathrm{d} m}{2 \pi} .
$$

Incorporating these modifications into equation 98 and neglecting a possible mass-dependence of the matrix element we obtain:

$$
\frac{\mathrm{d} \sigma_{34}^{12}}{\mathrm{~d} \Omega}=\frac{\left\langle p_{12}^{2}\right\rangle}{\left\langle p_{34}^{2}\right\rangle} \frac{\left(2 S_{1}+1\right)\left(2 S_{1}+1\right)}{\left(2 S_{3}+1\right)\left(2 S_{4}+1\right)} \times \sum_{J=J_{-}}^{J_{+}}\left\langle j_{1} m_{1} j_{2} m_{2} \| J M\right\rangle \frac{\mathrm{d} \sigma_{12}^{34}}{\mathrm{~d} \Omega} .
$$

Here, $S_{i}$ indicates the spin of particle $i$ and the summation of the Clebsch-Gordan-coefficients is over the isospin of the outgoing channel only. For the incoming channel, isospin is treated explicitly. The summation limits are given by:

$$
\begin{aligned}
& J_{-}=\max \left(\left|j_{1}-j_{2}\right|,\left|j_{3}-j_{4}\right|\right) \\
& J_{+}=\min \left(j_{1}+j_{2}, j_{3}+j_{4}\right)
\end{aligned} .
$$

The integration over the mass distributions of the resonances in equation (101) has been denoted by the brackets \langle\rangle , e.g.

$$
p_{3,4}^{2} \Rightarrow\left\langle p_{3,4}^{2}\right\rangle=\iint p_{C M S}^{2}\left(\sqrt{s}, m_{3}, m_{4}\right) A_{3}\left(m_{3}\right) A_{4}\left(m_{4}\right) \mathrm{d} m_{3} \mathrm{~d} m_{4}
$$


with the mass distribution $A_{r}(m)$ given by a free Breit-Wigner distribution with a mass-dependent width according to equation 96:

$$
A_{r}(m)=\frac{1}{N} \frac{\Gamma(m)}{\left(m_{r}-m\right)^{2}+\Gamma(m)^{2} / 4}
$$

with

$$
\lim _{\Gamma \rightarrow 0} A_{r}(m)=\delta\left(m_{r}-m\right)
$$

and the normalization constant

$$
N=\int_{-\infty}^{\infty} \frac{\Gamma(m)}{\left(m_{r}-m\right)^{2}+\Gamma(m)^{2} / 4} \mathrm{~d} m .
$$

Alternatively one can also choose a Breit-Wigner distribution with a fixed width, the normalization constant then has the value $N=2 \pi$.

The most frequent applications of equation (101) in UrQMD are the processes $\Delta_{1232} N \rightarrow N N$ and $\Delta_{1232} \Delta_{1232} \rightarrow N N$.

\section{Interface for thermal photon emission}

In the iEBE package, we provide a separate branch to allow users to perform calculations for electromagnetic probes from relativistic heavy-ion collisions. In Fig. 21, we illustrate the work flow of such integrated calculations.

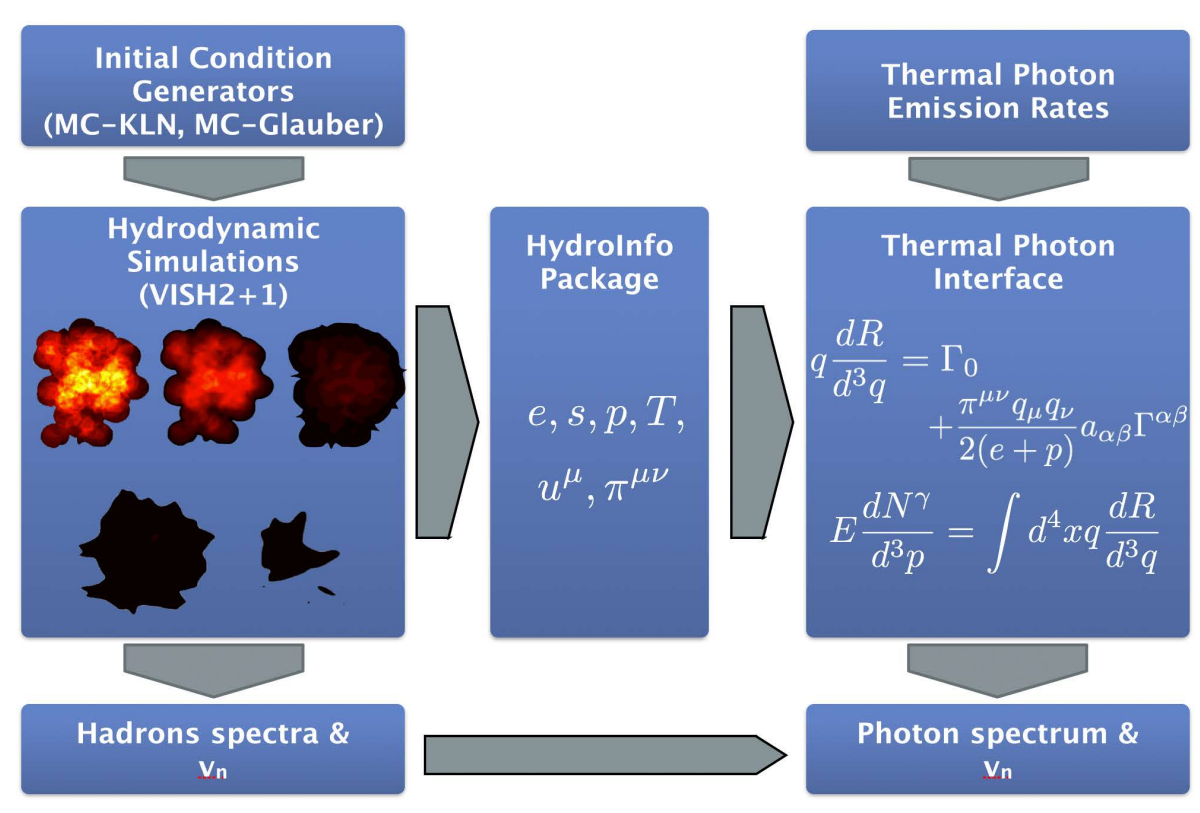

Figure 21: Work flow for event-by-event hydrodynamic simulation with photon emission. 
In order to compute thermal photon emission from an evolving viscous hydrodynamic medium, we need to output the evolution history of the local temperature, flow velocity and shear stress tensor and fold them with thermal photon emission rates to compute electromagnetic observables. Since the hydrodynamic evolution information is very demanding in terms of storage space, we output it in HDF5 binary format to minimize the storage requirement and increase the I/O efficiency. HDF5 format (Hierarchical Data Format) is a data mode $8^{8}$ library, and file format for storing and managing data. It supports an unlimited variety of datatypes, and is designed for flexible and efficient I/O and for high volume and complex data. The HDF5 library also provides multi-language support, which enables us to build our interface in both Fortran and C++ for future support.

The momentum spectrum of thermal photons emitted from the expanding fireball can be written as

$$
E \frac{d N^{\gamma}}{d^{3} p}=\int d^{4} x\left(\Gamma_{0}+\frac{\pi^{\mu \nu}}{2(e+P)} \Gamma_{\mu \nu}\right)
$$

where the integral goes over the space-time volume occupied by the radiating hot medium, $\Gamma_{0}$ is the thermal equilibrium emission rate, and the second term $\sim \pi_{\mu \nu}$ describes the shear viscous correction to the thermal emission rate. We can decompose $\Gamma_{\mu \nu}$ in a complete tensor basis and use the properties of the shear stress tensor, $\pi^{\mu \nu}=\Delta_{\alpha \beta}^{\mu \nu} \pi^{\alpha \beta}$, to write Eq. 107 in the form

$$
E \frac{d N^{\gamma}}{d^{3} p}=\int d^{4} x\left(\Gamma_{0}(u \cdot q, T)+\frac{\pi^{\mu v} \hat{q}_{\mu} \hat{q}_{v}}{2(e+P)} a_{\alpha \beta} \Gamma^{\alpha \beta}(u \cdot q, T)\right),
$$

where $\hat{q}^{\mu}=q^{\mu} /(u \cdot q), a_{\alpha \beta}$ is a projection operator:

$$
a_{\alpha \beta}=\frac{3}{2(u \cdot q)^{2}} q_{\alpha} q_{\beta}+u_{\alpha} u_{\beta}+g_{\alpha \beta}-\frac{3}{2(u \cdot q)}\left(q_{\alpha} u_{\beta}+q_{\beta} u_{\alpha}\right) .
$$

The use of tensor decomposition in Eq. 108 is particularly efficient numerically, because the anisotropic correction factors into a product of Lorentz scalars of which the first, $\pi^{\mu v} \hat{q}_{\mu} \hat{q}_{v}$, is most easily evaluated in the laboratory frame (where we know $\pi^{\mu \nu}$ from the solution of the hydrodynamic equations) while the second, $\Gamma_{1} \equiv a_{\alpha \beta} \Gamma^{\alpha \beta}$, is best worked out in the local rest frame of the fluid cell (where $u \cdot q$ reduces to the local rest frame energy of the photon). This helps to avoid performing extensive Lorentz boosts and 3-D rotations of $\pi^{\mu v}$ for each fluid cell when coupled to hydrodynamic simulations. Besides speeding-up the calculation, it allows us to tabulate the viscous corrections into one convenient table that can easily be used for phenomenological studies.

Please note that the work flow in Fig. 21 is generic for the calculation of all rare probes coupled to the evolving bulk medium that probe its temperature and flow velocity, such as jet energy loss and heavy quark diffusion. Similar modules for medium-induced jet quenching and jet shape modification as under construction.

\section{Conclusions}

In this work, we presented in detail the implementation of the iEBE-VISHNU package for event-by-event numerical simulations of relativistic heavy-ion collisions. We added multiplicity

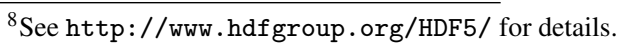


fluctuation in the MC-Glauber model based on the phenomenological KNO scaling observed in p-p collisions. This model can correctly reproduce the measured multiplicity distribution for $\mathrm{p}+\mathrm{Pb}$ collisions at $\sqrt{s_{\mathrm{NN}}}=5.02 \mathrm{TeV}$. The multiplicity fluctuations also change the initial eccentricity distribution, $\left\{\varepsilon_{n}\right\}$. In VISHNew, we improved the numerical stability of the $(2+1)$-d viscous hydrodynamic code when handling fluctuating initial conditions. We studied and documented the sensitivities of the final flow observables on the choice of the regulation strength parameters. VISHNew was tested against semi-analytical solutions derived based on Gubser's flow. The code iSS converts the fluid cells into individual particles according to the Cooper-Frye formula. We proposed three different ways of dealing with the negative probability issues related to generating Monte-Carlo samples for different simulation purposes and pointed out ways to minimize these problems in various settings. This document includes performance tests and numerical checks for all three methods of sampling thermally emitted particles.

In the present document we did not discuss the comparison of numerical results obtained with the iEBE-VISHNU code package for hadron and photon spectra and their anisotropy coefficients with experimental data. Such comparisons have been [70, 82] and will continue to be published elsewhere. Recent improvements of the code package include a module for pre-equilibrium evolution Landau-matched to viscous hydrodynamics [83], and the inclusion of bulk viscous effects as well as a module for the computation of Hanbury-Brown Twiss (HBT) two-particle correlations from the iEBE-VISHNU output are in progress. These and additional future improvements of the code package will be made available at https://u.osu.edu/vishnu/as soon as code testing of the new components is completed.

\section{Acknowledgments}

This work was supported in part by the U.S. Department of Energy, Office of Science, Office of Nuclear Physics, under Awards No. DE-SC0004286, DE-FG02-05ER41367, and (within the framework of the JET Collaboration) DE-SC0004104, and in part by the Natural Sciences and Engineering Research Council of Canada.. We gratefully acknowledge important contributions by Scott Moreland during the early stages of the development of the superMC module.

[1] Y. V. Kovchegov, E. Levin, Quantum chromodynamics at high energy, Cambridge monographs on particle physics, nuclear physics and cosmology, Cambridge Univ. Press, Cambridge, 2012.

[2] T. Epelbaum, F. Gelis, Pressure isotropization in high energy heavy ion collisions, Phys.Rev.Lett. 111 (2013) 232301. arXiv: 1307.2214 doi:10.1103/PhysRevLett.111.232301

[3] K. Dusling, T. Epelbaum, F. Gelis, R. Venugopalan, Role of quantum fluctuations in a system with strong fields: Onset of hydrodynamical flow, Nucl.Phys. A850 (2011)69-109. arXiv:1009.4363 doi:10.1016/j.nuclphysa. 2010.11.009

[4] H. Song, U. Heinz, Causal viscous hydrodynamics in $2+1$ dimensions for relativistic heavy-ion collisions, Phys. Rev. C 77 (2008) 064901. arXiv:0712.3715 doi:10.1103/PhysRevC.77.064901

[5] K. Dusling, D. Teaney, Simulating elliptic flow with viscous hydrodynamics, Phys. Rev. C 77 (2008) 034905. arXiv:0710.5932 doi:10.1103/PhysRevC.77.034905

[6] M. Luzum, P. Romatschke, Conformal Relativistic Viscous Hydrodynamics: Applications to RHIC results at $\sqrt{s_{\mathrm{NN}}}$ = 200 GeV, Phys. Rev. C78 (2008) 034915, [Erratum: Phys. Rev. C79, 039903 (2009)]. arXiv:0804.4015 doi:10.1103/PhysRevC.78.034915

[7] P. Bozek, Bulk and shear viscosities of matter created in relativistic heavy-ion collisions, Phys. Rev. C 81 (2010) 034909. arXiv:0911.2397 doi:10.1103/PhysRevC.81.034909

[8] H. Holopainen, H. Niemi, K. J. Eskola, Event-by-event hydrodynamics and elliptic flow from fluctuating initial state, Phys.Rev. C83 (2011) 034901. arXiv:1007.0368 doi:10.1103/PhysRevC.83.034901

[9] B. Schenke, S. Jeon, C. Gale, Elliptic and triangular flow in event-by-event (3+1)D viscous hydrodynamics, Phys.Rev.Lett. 106 (2011) 042301. arXiv: 1009.3244 doi:10.1103/PhysRevLett.106.042301

[10] L. Pang, Q. Wang, X.-N. Wang, Effects of initial flow velocity fluctuation in event-by-event (3+1)D hydrodynamics, Phys.Rev. C86 (2012) 024911. arXiv:1205.5019 doi:10.1103/PhysRevC.86.024911 
[11] I. Karpenko, P. Huovinen, M. Bleicher, A 3+1 dimensional viscous hydrodynamic code for relativistic heavy ion collisions, Comput.Phys.Commun. 185 (2014) 3016-3027. arXiv:1312.4160 doi:10.1016/j.cpc.2014. 07.010

[12] L. Del Zanna, V. Chandra, G. Inghirami, V. Rolando, A. Beraudo, et al., Relativistic viscous hydrodynamics for heavy-ion collisions with ECHO-QGP, Eur. Phys. J. C73 (2013) 2524. arXiv: 1305.7052 doi:10.1140/epjc/ s10052-013-2524-5

[13] M. Chojnacki, W. Florkowski, Temperature dependence of sound velocity and hydrodynamics of ultra-relativistic heavy-ion collisions, Acta Phys.Polon. B38 (2007) 3249-3262. arXiv:nucl-th/0702030

[14] H. Song, U. Heinz, Multiplicity scaling in ideal and viscous hydrodynamics, Phys. Rev. C 78 (2008) 024902. arXiv:0805.1756 doi:10.1103/PhysRevC.78.024902

[15] P. Huovinen, P. Petreczky, QCD Equation of State and Hadron Resonance Gas, Nucl. Phys. A837 (2010) 26-53 arXiv:0912.2541 doi:10.1016/j.nuclphysa.2010.02.015

[16] S. Borsanyi, Z. Fodor, C. Hoelbling, S. D. Katz, S. Krieg, et al., Full result for the QCD equation of state with $2+1$ flavors, Phys.Lett. B730 (2014) 99-104. arXiv:1309.5258 doi:10.1016/j.physletb.2014.01.007

[17] A. Bazavov, et al., Equation of state in ( 2+1 )-flavor QCD, Phys.Rev. D90 (9) (2014) 094503. arXiv: 1407.6387 doi:10.1103/PhysRevD.90.094503

[18] S. Bass, M. Belkacem, M. Bleicher, M. Brandstetter, L. Bravina, et al., Microscopic models for ultrarelativistic heavy ion collisions, Prog.Part.Nucl.Phys. 41 (1998) 255-369. arXiv: nucl-th/9803035 doi:10.1016/ S0146-6410(98)00058-1

[19] M. Bleicher, E. Zabrodin, C. Spieles, S. Bass, C. Ernst, et al., Relativistic hadron hadron collisions in the ultrarelativistic quantum molecular dynamics model, J.Phys. G25 (1999) 1859-1896. arXiv:hep-ph/9909407 doi: 10.1088/0954-3899/25/9/308

[20] T. Hirano, K. Tsuda, Collective flow and two pion correlations from a relativistic hydrodynamic model with early chemical freeze out, Phys. Rev. C 66 (2002) 054905. arXiv:nucl-th/0205043 doi:10.1103/PhysRevC.66. 054905

[21] H. Song, S. Bass, U. W. Heinz, Spectra and elliptic flow for identified hadrons in $2.76 \mathrm{~A} \mathrm{TeV} \mathrm{Pb+Pb} \mathrm{collisions,}$ Phys.Rev. C89 (2014) 034919. arXiv: 1311.0157 doi:10.1103/PhysRevC.89.034919

[22] U. W. Heinz, Towards the Little Bang Standard Model, J.Phys.Conf.Ser. 455 (2013) 012044. arXiv: 1304.3634 doi:10.1088/1742-6596/455/1/012044

[23] J. Noronha-Hostler, G. S. Denicol, J. Noronha, R. P. G. Andrade, F. Grassi, Bulk Viscosity Effects in Eventby-Event Relativistic Hydrodynamics, Phys. Rev. C88 (2013) 044916. arXiv:1305.1981 doi:10.1103/ PhysRevC.88.044916

[24] M. Habich, J. Nagle, P. Romatschke, Particle spectra and HBT radii for simulated central nuclear collisions of $\mathrm{C}+\mathrm{C}, \mathrm{Al}+\mathrm{Al}, \mathrm{Cu}+\mathrm{Cu}, \mathrm{Au}+\mathrm{Au}$, and $\mathrm{Pb}+\mathrm{Pb}$ from $\sqrt{s}=62.4-2760 \mathrm{GeV}$, Eur. Phys. J. C75 (2015) 15. arXiv: 1409.0040 doi:10.1140/epjc/s10052-014-3206-7

[25] S. Ryu, J. F. Paquet, C. Shen, G. Denicol, B. Schenke, et al., The importance of the bulk viscosity of QCD in ultrarelativistic heavy-ion collisions arXiv: 1502.01675

[26] B. Schenke, P. Tribedy, R. Venugopalan, Fluctuating Glasma initial conditions and flow in heavy ion collisions, Phys. Rev. Lett. 108 (2012) 252301. arXiv:1202.6646 doi:10.1103/PhysRevLett.108.252301

[27] C. Gale, S. Jeon, B. Schenke, P. Tribedy, R. Venugopalan, Event-by-event anisotropic flow in heavy-ion collisions from combined Yang-Mills and viscous fluid dynamics, Phys.Rev.Lett. 110 (2013) 012302. arXiv:1209.6330 doi:10.1103/PhysRevLett.110.012302

[28] H. Niemi, G. Denicol, P. Huovinen, E. Molnar, D. Rischke, Influence of a temperature-dependent shear viscosity on the azimuthal asymmetries of transverse momentum spectra in ultrarelativistic heavy-ion collisions, Phys. Rev. C86 (2012) 014909. arXiv:1203.2452 doi:10.1103/PhysRevC.86.014909

[29] E. Molnar, H. Holopainen, P. Huovinen, H. Niemi, Influence of temperature-dependent shear viscosity on elliptic flow at backward and forward rapidities in ultrarelativistic heavy-ion collisions, Phys. Rev. C90 (2014) 044904. arXiv: 1407.8152 doi:10.1103/PhysRevC.90.044904

[30] P. Bozek, Flow and interferometry in 3+1 dimensional viscous hydrodynamics, Phys.Rev. C85 (2012) 034901 arXiv:1110.6742 doi:10.1103/PhysRevC.85.034901

[31] P. Bozek, Collective flow in p-Pb and d-Pd collisions at TeV energies, Phys. Rev. C85 (2012) 014911. arXiv: 1112.0915 doi:10.1103/PhysRevC.85.014911

[32] L.-G. Pang, Y. Hatta, X.-N. Wang, B.-W. Xiao, Analytical and numerical Gubser solutions of the second-order hydrodynamics, Phys.Rev. D91 (7) (2015) 074027. arXiv:1411.7767 doi:10.1103/PhysRevD.91.074027

[33] Y. Akamatsu, S.-i. Inutsuka, C. Nonaka, M. Takamoto, A new scheme of causal viscous hydrodynamics for relativistic heavy-ion collisions: A Riemann solver for quark-gluon plasma, J. Comput. Phys. 256 (2014) 34-54. arXiv: 1302.1665 doi:10.1016/j.jcp.2013.08.047

[34] J. L. ALbacete, A. Dumitru, A model for gluon production in heavy-ion collisions at the LHC with rcBK unintegrated gluon densities $\operatorname{arXiv:1011.5161}$ 
[35] J. L. Albacete, A. Dumitru, H. Fujii, Y. Nara, CGC predictions for $\mathrm{p}+\mathrm{Pb}$ collisions at the LHC, Nucl.Phys. A897 (2013) 1-27. arXiv: 1209.2001 doi:10.1016/j.nuclphysa.2012.09.012

[36] H. Song, S. A. Bass, U. Heinz, Viscous QCD matter in a hybrid hydrodynamic+Boltzmann approach, Phys.Rev. C83 (2011) 024912. arXiv: 1012.0555 doi:10.1103/PhysRevC.83.024912

[37] W. Broniowski, M. Rybczynski, P. Bozek, GLISSANDO: Glauber initial-state simulation and more.., Comput.Phys.Commun. 180 (2009) 69-83. arXiv:0710.5731 doi:10.1016/j.cpc.2008.07.016

[38] B. Alver, M. Baker, C. Loizides, P. Steinberg, The PHOBOS Glauber Monte CarldarXiv: 0805. 4411

[39] C. Loizides, J. Nagle, P. Steinberg, Improved version of the PHOBOS Glauber Monte CarlcarXiv: 1408.2549

[40] D. Kharzeev, E. Levin, M. Nardi, The Onset of classical QCD dynamics in relativistic heavy ion collisions, Phys.Rev. C71 (2005) 054903. arXiv: hep-ph/0111315 doi:10.1103/PhysRevC.71.054903

[41] D. Kharzeev, E. Levin, M. Nardi, Color glass condensate at the LHC: Hadron multiplicities in pp, pA and AA collisions, Nucl.Phys. A747 (2005) 609-629. arXiv:hep-ph/0408050 doi:10.1016/j .nuclphysa. 2004 . 10.018

[42] U. Heinz, J. S. Moreland, Energy dependent growth of the nucleon and hydrodynamic initial conditions, Phys.Rev. C84 (2011) 054905. arXiv: 1108.5379 doi:10.1103/PhysRevC.84.054905

[43] P. Filip, Elliptic flow in central collisions of deformed nuclei, Phys.Atom.Nucl. 71 (2008) 1609-1618. arXiv: 0712.0088 doi:10.1134/S1063778808090172

[44] P. Filip, R. Lednicky, H. Masui, N. Xu, Initial eccentricity in deformed Au-197 + Au-197 and U-238 + U-238 collisions at $\mathrm{sNN}=200 \mathrm{GeV}$ at the BNL Relativistic Heavy Ion Collider, Phys.Rev. C80 (2009) 054903. doi: 10.1103/PhysRevC.80.054903

[45] T. Hirano, P. Huovinen, K. Murase, Y. Nara, Integrated Dynamical Approach to Relativistic Heavy Ion Collisions, Prog.Part.Nucl.Phys. 70 (2013) 108-158. arXiv:1204.5814 doi:10.1016/j.ppnp.2013.02.002

[46] D. Kharzeev, M. Nardi, Hadron production in nuclear collisions at rhic and high-density qcd Phys. Lett. B 507 (1-4) (2001) 121 - 128. doi:DOI: 10.1016/S0370-2693(01)00457-9

URL http://www.sciencedirect.com/science/article/B6TVN-430NSY5-H/2/ 7c9964b46166e2c8e5a1e1a451075f1e

[47] B. Back, et al., Centrality dependence of charged particle multiplicity at mid-rapidity in Au $+\mathrm{Au}$ collisions at $\sqrt{s_{N N}}$ = 130-GeV, Phys.Rev. C65 (2002) 031901. arXiv : nucl-ex/0105011 doi:10.1103/PhysRevC.65.031901

[48] T. Hirano, Y. Nara, Hydrodynamic afterburner for the color glass condensate and the parton energy loss, Nucl.Phys. A743 (2004) 305-328. arXiv:nucl-th/0404039 doi:10.1016/j.nuclphysa.2004.08.003

[49] T. Hirano, Y. Nara, Eccentricity fluctuation effects on elliptic flow in relativistic heavy ion collisions, Phys. Rev. C 79 (2009) 064904. arXiv: 0904.4080 doi:10.1103/PhysRevC.79.064904

[50] S. Chatrchyan, et al., Studies of azimuthal dihadron correlations in ultra-central $\mathrm{PbPb}$ collisions at $\sqrt{s_{N N}}=2.76$ TeV, JHEP 1402 (2014) 088. arXiv:1312.1845 doi:10.1007/JHEP02(2014) 088

[51] V. Khachatryan, et al., Charged particle multiplicities in $p p$ interactions at $\sqrt{s}=0.9,2.36$, and $7 \mathrm{TeV}$, JHEP 1101 (2011) 079. arXiv: 1011.5531 doi:10.1007/JHEP01(2011) 079

[52] R. Ansorge, et al., Charged Particle Multiplicity Distributions at 200-GeV and 900-GeV Center-Of-Mass Energy, Z.Phys. C43 (1989) 357. doi:10.1007/BF01506531

[53] S. Chatrchyan, et al., Multiplicity and transverse momentum dependence of two- and four-particle correlations in pPb and PbPb collisions, Phys.Lett. B724 (2013) 213-240. arXiv:1305.0609 doi:10.1016/j.physletb. 2013.06 .028

[54] B. B. Abelev, et al., Multiparticle azimuthal correlations in $\mathrm{p}-\mathrm{Pb}$ and $\mathrm{Pb}-\mathrm{Pb}$ collisions at the CERN Large Hadron Collider, Phys.Rev. C90 (5) (2014) 054901. arXiv: 1406.2474 doi:10.1103/PhysRevC.90.054901

[55] P. Bozek, W. Broniowski, Collective dynamics in high-energy proton-nucleus collisions, Phys. Rev. C88 (2013) 014903. arXiv: 1304.3044 doi:10.1103/PhysRevC.88.014903

[56] U. W. Heinz, H. Song, Causal relativistic hydrodynamics for viscous fluids, J.Phys. G35 (2008) 104126. arXiv: 0806.0352 doi:10.1088/0954-3899/35/10/104126

[57] C. Shen, U. Heinz, P. Huovinen, H. Song, Systematic parameter study of hadron spectra and elliptic flow from viscous hydrodynamic simulations of $\mathrm{Au}+\mathrm{Au}$ collisions at $\sqrt{s_{\mathrm{NN}}}=200 \mathrm{GeV}$, Phys. Rev. C 82 (2010) 054904 . arXiv:1010.1856 doi:10.1103/PhysRevC.82.054904

[58] S. S. Gubser, Symmetry constraints on generalizations of Bjorken flow, Phys.Rev. D82 (2010) 085027. arXiv: 1006.0006 doi:10.1103/PhysRevD.82.085027

[59] S. S. Gubser, A. Yarom, Conformal hydrodynamics in Minkowski and de Sitter spacetimes, Nucl.Phys. B846 (2011) 469-511. arXiv:1012.1314 doi:10.1016/j.nuclphysb.2011.01.012

[60] H. Marrochio, J. Noronha, G. S. Denicol, M. Luzum, S. Jeon, et al., Solutions of Conformal Israel-Stewart Relativistic Viscous Fluid Dynamics, Phys.Rev. C91 (1) (2015) 014903. arXiv:1307.6130 doi:10.1103/ PhysRevC.91.014903

[61] H. Niemi, G. Denicol, How large is the Knudsen number reached in fluid dynamical simulations of ultrarelativistic heavy ion collisions arXiv: 1404.7327 
[62] P. F. Kolb, U. W. Heinz, Hydrodynamic description of ultrarelativistic heavy ion collisions arXiv: nucl-th/ 0305084

[63] Y. Yariv, Z. Fraenkel, Intranuclear cascade calculation of high-energy heavy-ion interactions, Phys.Rev. C20 (1979) 2227-2243. doi:10.1103/PhysRevC.20.2227,10.1103/PhysRevC.21.2139

[64] H. Kruse, B. Jacak, J. Molitoris, G. Westfall, H. Stoecker, Vlasov-Uehling-Uhlenbeck theory of medium-energy heavy ion reactions: role of mean field dynamics and two-body collisions, Phys.Rev. C31 (1985) 1770-1774. doi:10.1103/PhysRevC.31.1770

[65] J. Aichelin, A. Bohnet, G. Peilert, H. Stoecker, W. Greiner, et al., Quantum Molecular Dynamics Approach to Heavy Ion Collisions: Description of the Model, Comparison With Fragmentation Data, and the Mechanism of Fragment Formation, Phys.Rev. C37 (1988) 2451-2468. doi : 10.1103/PhysRevC .37.2451

[66] H. Sorge, H. Stoecker, W. Greiner, Poincare Invariant Hamiltonian Dynamics: Modeling Multi - Hadronic Interactions in a Phase Space Approach, Annals Phys. 192 (1989) 266-306. doi:10.1016/0003-4916(89) 90136-X

[67] R. M. Barnett, et al., Review of particle physics. Particle Data Group, Phys.Rev. D54 (1996) 1-720. doi:10. 1103/PhysRevD.54.1

[68] M. Belkacem, M. Brandstetter, S. Bass, M. Bleicher, L. Bravina, et al., Equation of state, spectra and composition of hot and dense infinite hadronic matter in a microscopic transport model, Phys.Rev. C58 (1998) 1727-1733. arXiv:nucl-th/9804058 doi:10.1103/PhysRevC.58.1727

[69] P. Danielewicz, G. Bertsch, Production of deuterons and pions in a transport model of energetic heavy ion reactions, Nucl.Phys. A533 (1991) 712-748. doi:10.1016/0375-9474(91)90541-D

[70] Z. Qiu, C. Shen, U. Heinz, Hydrodynamic elliptic and triangular flow in Pb-Pb collisions at $\sqrt{s}=2.76 \mathrm{ATeV}$, Phys. Lett. B707 (2012) 151-155. arXiv:1110.3033 doi:10.1016/j.physletb.2011.12.041

[71] U. Heinz, Z. Qiu, C. Shen, Fluctuating flow angles and anisotropic flow measurements, Phys. Rev. C87 (2013) 034913. arXiv:1302.3535 doi:10.1103/PhysRevC.87.034913

[72] C. Shen, U. W. Heinz, J.-F. Paquet, C. Gale, Thermal photons as a quark-gluon plasma thermometer reexamined, Phys.Rev. C89 (4) (2014) 044910. arXiv: 1308.2440 doi:10.1103/PhysRevC.89.044910

[73] C. Shen, U. W. Heinz, J.-F. Paquet, I. Kozlov, C. Gale, Anisotropic flow of thermal photons as a quark-gluon plasma viscometer, Phys. Rev. C91 (2015) 024908. arXiv:1308.2111 doi:10.1103/PhysRevC.91.024908

[74] U. W. Heinz, J. Liu, C. Shen, Electromagnetic fingerprints of the Little Bang, Nucl. Phys. A932 (2014) $310-317$. arXiv:1403.8101

[75] C. Shen, U. Heinz, J.-F. Paquet, C. Gale, Thermal photon anisotropic flow serves as a quark-gluon plasma viscometer in: Proceedings, 6th International Conference on Hard and Electromagnetic Probes of High-Energy Nuclear Collisions (Hard Probes 2013), 2014. arXiv:1403.7558 URL http://inspirehep.net/record/1287938/files/arXiv:1403.7558.pdf

[76] C. Shen, J.-F. Paquet, J. Liu, G. Denicol, U. Heinz, C. Gale, Event-by-event direct photon anisotropic flow in relativistic heavy-ion collisions, Nucl. Phys. A931 (2014) 675-680. arXiv:1407.8533 doi:10.1016/j. nuclphysa.2014.08.030

[77] C. Shen, Z. Qiu, U. Heinz, Shape and flow fluctuations in ultra-central Pb+Pb collisions at the LHC, Phys. Rev. C92 (2015) in press. arXiv: 1502.04636

[78] C. Plumberg, U. Heinz, Interferometric signatures of the temperature dependence of the specific shear viscosity in heavy-ion collisions, Phys. Rev. C91 (2015) 054905. arXiv:1503.05605 doi:10.1103/PhysRevC.91. 054905

[79] C. Shen, J. F. Paquet, G. Denicol, S. Jeon, C. Gale, Thermal photon radiation in high multiplicity p+Pb collisions at the Large Hadron ColliderarXiv: 1504.07989

[80] J. E. Bernhard, P. W. Marcy, C. E. Coleman-Smith, S. Huzurbazar, R. L. Wolpert, et al., Quantifying properties of hot and dense QCD matter through systematic model-to-data comparison, Phys. Rev. C91 (2015) 054910. arXiv: 1502.00339 doi:10.1103/PhysRevC.91.054910

[81] A. Goldschmidt, Z. Qiu, C. Shen, U. Heinz, Collision Geometry and Flow in Uranium+Uranium Collisions arXiv : 1502.00603

[82] A. Goldschmidt, Z. Qiu, C. Shen, U. Heinz, Collision Geometry and Flow in Uranium+Uranium Collisions arXiv: 1507.03910

[83] J. Liu, C. Shen, U. W. Heinz, Pre-equilibrium evolution effects on heavy-ion collision observables, Phys. Rev. C91 (2015) in press. arXiv: 1504.02160 